\title{
A comprehensive review of deep learning-based single image super-resolution
}

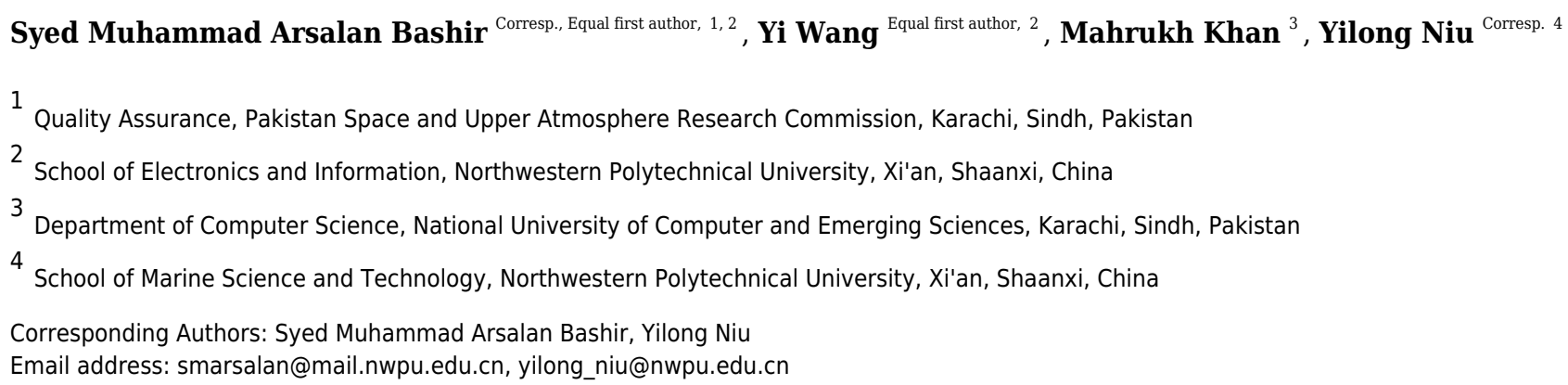

Image super-resolution (SR) is one of the vital image processing methods that improve the resolution of an image in the field of computer vision. In the last two decades, significant progress has been made in the field of super-resolution, especially by utilizing deep learning methods. This survey is an effort to provide a detailed survey of recent progress in single-image super-resolution in the perspective of deep learning while also informing about the initial classical methods used for image super-resolution. The survey classifies the image SR methods into four categories, i.e., classical methods, supervised learningbased methods, unsupervised learning-based methods, and domain-specific SR methods. We also introduce the problem of SR to provide intuition about image quality metrics, available reference datasets, and SR challenges. Deep learning-based approaches of SR are evaluated using a reference dataset. Some of the reviewed state-of-the-art image SR methods include enhanced deep SR network (EDSR), cycle-in-cycle GAN (CinCGAN), multiscale residual network (MSRN), meta residual dense network (Meta-RDN), recurrent back-projection network (RBPN), second-order attention network (SAN), SR feedback network (SRFBN) and wavelet-based residual attention network (WRAN). Finally, this survey is concluded with future directions and trends in SR and open problems in SR to be addressed by the researchers. 


\section{A comprehensive review of deep learning-based 2 single image super-resolution}

3

4 Syed Muhammad Arsalan Bashir ${ }^{1,2}$, Yi Wang ${ }^{2}$, Mahrukh Khan ${ }^{3}$, Yilong Niu ${ }^{4}$

5

$6 \quad{ }^{1}$ Quality Assurance, Pakistan Space and Upper Atmosphere Research Commission, Karachi, 7 Sindh, Pakistan.

$8{ }^{2}$ School of Electronics and Information, Northwestern Polytechnical University, Xi' an, Shaanxi, 9 China

$10{ }^{3}$ Department of Computer Science, National University of Computer and Emerging Sciences, 11 Karachi, Sindh, Pakistan

$12{ }^{4}$ School of Marine Science and Technology, Northwestern Polytechnical University, Xi'an, 13 Shaanxi, China

15 Corresponding Authors:

16 Yilong Niu and Syed Muhammad Arsalan Bashir

17 Email address: yilong_niu@nwpu.edu.cn; smarsalan@mail.nwpu.edu.cn 18 
19

20

21

22

23

24

25

26

27

28

29

30

31

32

33

34

35

36

37

38

39

40

41

42

43

44

45

46

47

48

49

50

51

52

53

54

55

56

57

58

\section{Abstract}

Image super-resolution (SR) is one of the vital image processing methods that improve the resolution of an image in the field of computer vision. In the last two decades, significant progress has been made in the field of super-resolution, especially by utilizing deep learning methods. This survey is an effort to provide a detailed survey of recent progress in single-image super-resolution in the perspective of deep learning while also informing about the initial classical methods used for image super-resolution. The survey classifies the image SR methods into four categories, i.e., classical methods, supervised learning-based methods, unsupervised learning-based methods, and domain-specific SR methods. We also introduce the problem of SR to provide intuition about image quality metrics, available reference datasets, and SR challenges. Deep learning-based approaches of SR are evaluated using a reference dataset. Some of the reviewed state-of-the-art image SR methods include enhanced deep SR network (EDSR), cyclein-cycle GAN (CinCGAN), multiscale residual network (MSRN), meta residual dense network (Meta-RDN), recurrent back-projection network (RBPN), second-order attention network (SAN), SR feedback network (SRFBN) and wavelet-based residual attention network (WRAN). Finally, this survey is concluded with future directions and trends in SR and open problems in SR to be addressed by the researchers.

\section{Introduction}

The image-based computer graphics models lack resolution independence (Freeman, Jones \& Pasztor, 2002) as the images cannot be zoomed beyond the image sample resolution without compromising the quality of images. This is the case, especially in realistic images, for instance, natural photographs. Thus, simple image interpolation will lead to the blurring of features and edges within a sample image.

The concept of super-resolution was first used by (Gerchberg, 1974) to improve the resolution of an optical system beyond the diffraction limit. In the past two decades, the concept of superresolution (SR) is defined as the method of producing high-resolution (HR) images from a corresponding low-resolution (LR) image. Initially, this technique was classified as spatial resolution enhancement (Tsai \& Huang, 1984). The applications of super-resolution include computer graphics (Kim, Lee \& Lee, 2016a,b; Tao et al., 2017), medical imaging (Bates et al., 2007; Fernández-Suárez \& Ting, 2008; Huang et al., 2008; Hamaide et al., 2017; Jurek et al., 2020; Teh et al., 2020; Bashir \& Wang, 2021a), security, and surveillance (Zhang et al., 2010; Shamsolmoali et al., 2018; Lee, Kim \& Heo, 2020), which shows the importance of this topic in recent years.

Although being explored for decades, image super-resolution remains a challenging task in computer vision. This problem is fundamentally ill-posed because there can be several HR images with slight variations in camera angle, color, brightness, and other variables for any given LR image. Furthermore, there are fundamental uncertainties among the LR and HR data since the downsampling of different HR images may lead to a similar LR image, making this conversion a many-to-one process (Yang \& Yang, 2013). 
59 The existing methods of image super-resolution can be categorized into single-image super-

60

61

62

63

64

65

66

67

68

69

70

71

72

73

74

75

76

77

78

79

80

81

82

83

84

85

86

87

88

89

90

91

92

93

94

95

96

97

98

resolution (SISR) and multiple-image approaches. In single image SR, the learning is performed for single LR-HR pair for a single image, while in multiple-image SR, the learning is performed for a large number of LR-HR pairs for a particular scene, thereby enabling the generation of an HR image from a scene (multiple images) (Kawulok et al., 2020). Video super-resolution deals with multiple successive images (frames) and utilizes the relationship within the frames to superresolve a target frame; it is a special type of multiple image SR where the images are part of a scene containing different frames (Liu et al., 2020b).

In the past, classical SR methods such as statistical methods, prediction-based methods, patchbased methods, edge-based, and sparse representation methods were used to achieve superresolution. However, recently the advances in computational power and big data have made researchers use deep learning (DL) to address the problem of SR. In the past decade, deep learning-based SR studies have reported superior performance than the classical methods, and DL methods have been used frequently to achieve SR. Researchers have used a range of methods to explore SR, ranging from the first method of Convolutional Neural Network (CNN) (Dong et al., 2014) to the recently used Generative Adversarial Nets (GAN) (Ledig et al., 2017). In principle, the methods used in deep learning-based SR methods vary in hyper-parameters such as network architecture, learning strategies, activation functions, and loss functions.

In this study, a brief overview of the classical methods of SR is outlined initially, whereas the main focus is given to give an overview of the most recent research in SR using deep learning. Previous studies have explored the literature on SR, but most of these studies emphasize the classical methods (Borman \& Stevenson, 1998; Park, Park \& Kang, 2003; van Ouwerkerk, 2006; Yang, Ma \& Yang, 2014; Thapa et al., 2016), additionally (Yang, Ma \& Yang, 2014; Thapa et al., 2016) used human visual perception to gauge the performance of SR methods.

In recent years, there are some reviews (Ha et al., 2019; Yang et al., 2019; Zhang et al., 2019c; Zhou \& Feng, 2019; Li et al., 2020) focused on deep learning-based image super-resolution. The study by Yang et al. (Yang et al., 2019) was focused on the deep learning methods for single image super-resolution Zhang et al. (Zhang et al., 2019c) limited the scope of image SR to CNN-based methods for space applications, thereby only reviewing four methods namely, SRCNN, FSRCNN, VDSR, and DRCN. Ha et al. (Ha et al., 2019) reviewed the state-of-the-art SISR methods and classified them based on the type of framework, i.e., CNN, RNN-CNN-based methods, and GAN-based methods. Zhou et al. (Zhou \& Feng, 2019) briefly reviewed some of the state-of-the-art SISR methods and provided an introduction of some of the methods without any evaluation of comparison of methods, while Li et al. (Li et al., 2020) reviewed the state-ofthe-art methods in image SR while emphasizing on the methods based on CNNs and GANs for real-time applications. These review papers did not encompass the domain of super-resolution as a whole, and this paper fills that research gap by providing an overview of both classical and deep learning-based methods. At the same time, we have reviewed the deep learning-based methods into subdomain based on the functional blocks, i.e., upsampling methods, SR networks, learning strategies, SR framework and other improvements. This review paper fills the gap of a

Peer] Comput. Sci. reviewing PDF | (CS-2021:02:58423:2:0:NEW 3 Jun 2021) 
99

100

101

102

103

104

105

106

107

108

109

110

111

112

113

114

115

116

117

118

119

120

121

122

123

124

125

126

127

128

129

130

131

132

133

134

135

136

137

comprehensive review where a reader could access the overall progress of image super-

resolution with appropriate section for the overall image quality metrics, SR methods, datasets, applications, and challenges in the field of image SR.

This survey is a comprehensive overview of the recent advances in SR, emphasizing deep

learning-based approaches and their achievements in systematically achieving SR. Table S1 and

Table $\mathrm{S} 2$ respectively show the complete list of symbols and acronyms used in this study. The key features of this study are:

1. We highlight the brief overview of the classical methods in SR and their contributions in light of past studies to give perspective.

2. We provide a detailed survey of deep learning-based SR, including the definition of the problem, dataset details, performance evaluation, deep learning methods used for SR, and specific applications where these SR methods were used and their performance.

3. We compare and contrast the recent advances in deep learning-based SR methods by summarizing the bounds of the methods by providing details of components of the SR methods used structurally.

4. Finally, the open problems in SR and critical challenges that require further probing are highlighted in this survey to provide future directions in SR.

This study is organized as follows:

In Section 1, we have introduced the concept of SR and the overall overview of this study. In Fig. 1, we have summarized the hierarchical structure of this review. There are four main sections: classical methods, deep learning-based methods, applications of SR, Discussion, and future directions. In Section 2, we put forward the problem definition and details of the evaluation dataset. Section 3 discusses the methodology for the selection of studies included within this review. In Section 4, we compare and contrast the classical methods of SR, whereas, in Section 5, the SR methods based on supervised deep learning are explored. Section 6 covers the studies that used unsupervised deep learning-based methods for SR, and in Section 7, various field-specific applications of SR in recent years are discussed. Section 8 summarizes open challenges and limitations in current SR methods and puts forward future research directions, while Section 9 highlights the conclusions.

\section{Super-resolution: Definitions and Terminologies}

In this section, the problem definition and the associated concepts of image super-resolution are discussed in light of the literature review.

\subsection{Single Image Super-resolution - Problem Definition}

The image SR focuses on the recovery of an HR image from LR image input as and in principle, the LR image $I_{x L R}$ can be represented as the output of the degradation function, as shown in (1).

$$
I_{x L R}=d\left(I_{y H R}, \partial\right)
$$


138 Where $d$ is the SR degradation function that is responsible for the conversion of HR image to LR

139

140

141

142

143

144

145

146

147

148

149

150

151

152

153

154

155

156

157

158

159

160

161

162

163

164

165

166

167

168

169

170

image, $I_{y H R}$ is the input HR image (reference image), whereas $\partial$ depicts the input parameters of

the image degradation function. Degradation parameters are usually scaling factor, blur type, and noise. In practice, the degradation process and dependent parameters are unknown, and only LR images are used to get HR images by the SR method. The SR process is responsible for

predicting the inverse of the degradation function $\mathrm{d}$, such that $g=d^{-1}$

$$
g\left(I_{x L R}, \delta\right)=d^{-1}\left(I_{x H R}\right)=I_{y E} \approx I_{y H R}
$$

Where $g$ is the SR function, $\delta$ depicts the input parameters to the function $g$, and $I_{y E}$ is the estimated HR corresponding to the input $I_{x L R}$ image. It is also worth noticing that the superresolution function, as in (2), is ill-posed, as the function $g$ is a non-injective function; thus, there are infinite possibilities of $I_{y E}$ for which the condition $d\left(I_{y E}, \partial\right)=I_{x L R}$ will hold.

The degradation process for the input LR images is unknown, and this process is affected by numerous factors such as sensor-induced noise, artifacts created because of lossy compression, speckle noise, motion blur, and misfocused images. In the literature, most of the studies have used a single downsampling function as the image degradation function:

$$
d\left(I_{y H R}, \partial\right)=\left(I_{y H R}\right) \downarrow_{s_{f}},\{s\} \subseteq \partial
$$

Where $\downarrow_{s_{f}}$ is the downsampling operator with $s_{f}$ being the scaling factor. One of the frequently used downsampling functions in SR is the bicubic interpolation (Shi et al., 2016; Zhang \& An, 2017; Shocher, Cohen \& Irani, 2018) with antialiasing. In some studies, like (Zhang, Zuo \& Zhang, 2018), researchers have used more operations in the downsampling function, and the overall downsampling operation is:

$$
d\left(I_{y H R}, \partial\right)=\left(I_{y H R} \otimes \kappa\right) \downarrow_{s_{f}}+n_{\sigma},\{\kappa, s, \sigma\} \subseteq \partial
$$

Where $I_{y H R} \otimes \kappa$ depicts the convolution of the HR image $I_{y H R}$ with the blurring kernel $\kappa, n_{\sigma}$ represents the additive white Gaussian noise with a standard deviation of $\sigma$. The degradation function defined in (4) and Fig. 2 is closer to the actual function as it considers more parameters than the simple downsampling degradation function (Zhang, Zuo \& Zhang, 2018).

Finally, the purpose of SR is to minimize the loss function as follows:

$$
\hat{\phi}=\left[\min L\left(I_{y E}, I_{y H R}\right)\right]_{\phi}+h \Psi(\phi)
$$

Where $\mathrm{L}\left(I_{y E}, I_{y H R}\right)$ is the loss function between the output HR image of SR and the actual HR image, $h$ is the tradeoff parameter, whereas $\Psi(\phi)$ is the regularization term. The most common loss function used in SR is the pixel-based mean square error (MSE), which can also be referred to as pixel loss. In recent years, researchers have used a combination of various loss functions, 
171 and these combinations are further explored in later sections. Further mathematical modeling of 172 the SR problem is discussed in (Candès \& Fernandez-Granda, 2014).

173

174

175

176

177

178

179

180

181

182

183

184

185

186

187

188

189

190

191

192

193

\subsection{Methods for quality of $S R$ images}

Image quality can have several definitions as per the measurement methods, and it is generally a measure of the quality of visual attributes and perception of the viewers. The image quality assessment (IQA) methods are characterized into subjective methods (human perception of an image is natural and of good quality) and objective methods (quantitative methods by which image quality can be numerically computed) (Thung \& Raveendran, 2009).

Quality-related visual aspects of an image are mostly a good measure, but this method requires more resources, especially if the dataset is large (Wei, Yuan \& Cai, 1999); thus, in SR and computer vision tasks, the more suitable methods are objective. As per (Saad, Bovik \& Charrier, 2012), the IQA methods are primarily categorized into three categories, i.e., reference imagebased features from the actual image and blind IQA with no information about the ground truth. In this section, IQA methods primarily used in the domain of SR are further explored.

\subsubsection{Peak Signal-to-Noise Ratio}

In information systems, the peak signal-to-noise ratio (PSNR) is a measurement technique for analyzing the signal power compared to the noise power, especially in images; the PSNR is used as a quantitative measure of the compression quality of an image. In super-resolution, the PSNR of an image is defined by the maximum pixel value and the mean square error between the reference image and the SR image, also known as the power of image distortion noise. For a given maximum pixel value $(M)$ and the reference image $\left(I_{r}\right)$ having t pixels and the SR image $\left(I_{y}\right)$, the peak signal-to-noise ratio is defined as:

$$
P S N R=10 \log _{10}\left(\frac{M^{2}}{M S E}\right)
$$

Where $\mathrm{M}$ is mostly for 8-bit color space depth, i.e., the max value of 255 and MSE is given by:

$$
M S E=\frac{1}{t} \sum_{i=1}^{t}\left(I_{r}(i)-I_{y}(i)\right)^{2}
$$

198

199

200

201

202

203

204

205

206

207

As seen from (6), the PSNR is related to the individual pixel intensity values of the SR image and reference image and is a pixel-based metric of image quality. In some cases (Almohammad \& Ghinea, 2010; Horé \& Ziou, 2010; Goyal, Lather \& Lather, 2015), this quality metric can be misleading as the overall image might not be visually similar to that of the reference image. This metric is still used for image comparisons, especially comparing the results of SR algorithms with previously published results to compare the working of any new method in the field of SR. MSE for color images averaged for color channels, and an alternate approach is to measure PSNR for luminance and or greyscale channels separately as the human eye is more sensitive to changes in luminance in contrast to changes in chrominance (Dabov et al., 2006). 
208

209

210

211

212

213

214

215

216

217

218

219

220

221

222

223

224

225

226

227

228

229

230

\subsubsection{Structural Similarity Index}

The visual perception of humans is efficient in extracting the structural information within an image, and PSNR does not consider the structural composition of the image (Rouse \& Hemami, 2008). The structural similarity index metric (SSIM) was proposed by (Wang et al., 2004) to measure the structural similarity between images by comparing the contrast, luminance, and structural details within the reference image.

An image $I_{r}$ with total pixels $P$; the contrast $C_{I}$, and luminance $L_{I}$ can be denoted as the standard deviation and the mean of the image intensity given by:

$$
L_{I}=\frac{1}{M} \sum_{i=1}^{M} I_{r}(i)
$$

The $i^{t h}$ pixel of the reference image is denoted by $I_{r}(i)$. The comparisons based on the contrast and luminance between the reference image $I_{r}$ and the estimated image $\hat{I}$ are:

$$
C_{I}=\sqrt{\left(\frac{1}{M-1} \sum_{i=1}^{M}\left(I_{r}(i)-L_{I}\right)^{2}\right)}
$$

Where $\mu_{1}=\left(k_{1} S\right)^{2}$ and $\mu_{2}=\left(k_{2} S\right)^{2}$, these constant terms ensure stability by ensuring $k_{1}<<1$ and $k_{2}<<1$.

Normalized pixel values $I_{r}-L_{I_{r}} / C_{I_{r}}$ represent the image structure, while the inner product of these is the equivalent of structural similarity between the reference image $I_{r}$ and the estimated image $\hat{I}$. The covariance $\sigma_{I_{r},}$, is given by:

$$
\sigma_{I_{r}, \hat{I}}=\frac{1}{M-1} \sum_{i=1}^{M}\left(I_{r}(i)-L_{I_{r}}\right)\left(\hat{I}(i)-L_{\hat{I}}\right)
$$

Function for structural comparison $\operatorname{Com}_{s}\left(I_{r}, \hat{I}\right)$ is given by:

$$
\operatorname{Com}_{s}\left(I_{r}, \hat{I}\right)=\frac{\sigma_{I_{r}, \hat{I}}+\mu_{3}}{C_{I_{r}} C_{\hat{I}}+\mu_{3}}
$$

Where $\mu_{3}$ is stability constant, the final structural similarity index (SSIM) is given by: 


$$
\operatorname{SSIM}\left(I_{r}, \hat{I}\right)=\left\{\operatorname{Com}_{l}\left(I_{r}, \hat{I}\right)\right\}^{\alpha}\left\{\operatorname{Com}_{c}\left(I_{r}, \hat{I}\right)\right\}^{\beta}\left\{\operatorname{Com}_{s}\left(I_{r}, \hat{I}\right)\right\}^{\gamma}
$$

233 The control parameters $\alpha, \beta$, and $\gamma$ can be adjusted to increase the importance of luminance,

234 contrast, and structural comparison in calculating the SSIM .

235 Conventionally, PSNR is used in computer vision tasks for evaluation, but SSIM is based on

236 human perception of structural information within an image. Thus this method is widely used for

237 comparing the structural similarity between images (Blau \& Michaeli, 2018; Sara, Akter \&

238 Uddin, 2019). In medical images where the variance or luminance of the reference images are

239 low, SSIM could be very unstable, thus reporting false results; however, this is not the case for

240

241

242

243

244

245

246

247

248

249

250

251

252

253

254

255

256

257

258

259

260

261

262

263

264

265

266

267

268

269

270 natural images (Pambrun \& Noumeir, 2015).

\subsubsection{Opinion Scoring}

Opinion scoring is a qualitative method, which lies in the subjective category of IQA. In this method, the quality testers are asked to grade the quality of images based on specific criteria, e.g., sharpness, natural look, and color, where the final graded score is the mean of the rated scores.

This method has limitations such as non-linearity between the scores, variation in results due to changes in test criteria, and human error. In SR, certain methods have reported good objective quality scores but scored poorly in subjective results, especially in human face reconstruction (Ledig et al., 2017), (Nasrollahi \& Moeslund, 2014; Chen et al., 2018b). Thus, the opinion scoring method is also used in studies (Wei, Yuan \& Cai, 1999), (Deng, 2018; Ravì et al., 2018, 2019; Vasu, Thekke Madam \& Rajagopalan, 2019) to measure the quality of human perception.

\subsubsection{Perceptual Quality}

Opinion scoring used human raters for manual evaluation of the images; while this method can provide accurate results as far as human perception is concerned, this method requires many resources, especially large datasets (Viswanathan \& Viswanathan, 2005). Initially, (Kim \& Lee, 2017) proposed a CNN-based full reference image quality assessment (FR-IQA) model where human behavior was learned using an IQA database that contained distorted images, subjective scores, and error maps, and this method was called DeepQA.

In (Ma et al., 2017a), the authors used quality-discriminable image pairs (DIP) for training, and the system was called dipQA (DIP inferred quality index); they used RankNet with L2R algorithm to learn blind opinion IQA, whereas in (Ma et al., 2018) a multi-task end-to-end optimized deep neural network (MEON) was proposed. MEON used two stages, in the first stage, distortion type learning using large datasets already available, and in the second stage, the output of the first stage was used to train the quality assessment network using stochastic gradient descent. In (Talebi \& Milanfar, 2018), the authors used CNN to develop a no-reference IQA method known as NIMA; NIMA was trained on pixel-level and aesthetic quality datasets. RankIQA (Liu, Weijer \& Bagdanov, 2017) trained a Siamese network to grade the quality of images using datasets with known image distortions, CNNs were used to learn the IQA, and this 
271 method even outperformed full-reference methods without using the reference image. IQA

272 proposed in (Bosse et al., 2018) included ten convolution layers and five pooling layers for

273 feature extraction while there were two fully connected layers for regression; this method

274 performed significantly well for both no-reference and full-reference IQA.

275 Even though opinion scoring and perceptual quality-based methods do exhibit human perception

276 in IQA, but the quality we require is still an open question (i.e., if we want images to be more

277 natural or similar to the reference image); thus, PSNR and SSIM are the primarily used methods

278 in computer vision and SR.

279

280

\subsubsection{Task-based Evaluation}

281

282

283

284

285

286

287

288

289

290

291

292

293

294

295

296

297

298

299

300

301

302

303

304

305

306

307

308

309

310

Although the primary purpose of image SR is to achieve better resolution, as mentioned earlier, SR is also helpful in other computer vision tasks (Kim, Lee \& Lee, 2016b), (Tao et al., 2017), (Teh et al., 2020), (Liu et al., 2018a). The performance achieved in these can indirectly measure the performance of the SR methods used in those tasks. In the case of medical images, the researchers used the original and SR constructed images to see the performance in the training and prediction phases. In general, computer vision tasks such as classification (Krizhevsky, Sutskever \& Hinton, 2012; Cai et al., 2019), face recognition (Nasrollahi \& Moeslund, 2014; Liu et al., 2015; Chen et al., 2018b), and object segmentation (Martin et al., 2001; Lin et al., 2014; Wang et al., 2018b) can be done using SR images. The performance of these computer vision tasks can be used as a metric to assess the performance of the SR method.

\subsubsection{Miscellaneous IQA Methods}

The development of IQA methods is an open field, and in recent years various researchers have proposed SR metrics, but these methods were not used widely by the SR community. Feature similarity (FSIM) index metric (Zhang et al., 2011) evaluates image quality by extracting feature points considered by the human visual system based on gradient magnitude and phase congruency. The multi-scale structural similarity (MS-SSIM) (Wang, Simoncelli \& Bovik, 2003) used multi-scale to incorporate variations in the viewing conditions to measure the image quality and proposed that MS-SSIM provides form flexibility in the measurement of image quality than single-scale SSIM. In (Li \& Bovik, 2010), the authors claimed that SSIM and MS-SSIM do not perform well on distorted and noisy images; thus, they used a four-component-based weighted method that adjusted the weight of scores based on the local feature, whereas in the case of contrast-distorted images (Yao \& Liu, 2018) like TID2013 and CSIQ datasets SSIM does not perform well.

According to (Blau \& Michaeli, 2018), the perceptual quality and image distortion are at odds with each other; as the distortion decreases, the perceptual quality should also be worse; thus, the accurate measurement of SR image quality is still an open area of research.

The comparison of image quality assessment metrics for super-resolution is shown in Table 1. It depends on the requirements of the methods; most of the methods use PSNR and SSIM to evaluate the performance as these are quantitative methods.

Peer) Comput. Sci. reviewing PDF | (CS-2021:02:58423:2:0:NEW 3 Jun 2021) 


\section{2.3. Operating Color Channels}

312 In most datasets, RGB color space is used; thus, SR methods mostly employ RGB images,

$313 \mathrm{YCbCr}$ space is also used in SR (Dong et al., 2016). The $\mathrm{Y}$ component in $\mathrm{YCbCr}$ is the

314 luminance component, which represents the light intensity, while $\mathrm{Cb}$ and $\mathrm{Cr}$ are the chrominance 315 components (i.e., blue-differenced and red-differenced Chroma channels) (Shaik et al., 2015). In

316 recent years, most of the SR challenges and datasets use the RGB color space, limiting the use of 317 RGB space for comparison with state of the art. Furthermore, the results of IQA based on PSNR

318 vary if the color space in the testing stage is different from the training/evaluation stage.

319

320

\subsection{Details of the reference dataset}

321

322

The datasets used in evaluating the SR algorithms are summarized in this section; the various

323 datasets discussed in this section vary in the total number of example images, image resolution, quality, and imaging hardware setup. A few of the datasets comprise paired LR-HR images for training and testing SR algorithms. In contrast, the rest of the datasets include HR images, and the corresponding LR images are usually generated by using bicubic interpolation with antialiasing as performed in (Shi et al., 2016; Zhang \& An, 2017; Shocher, Cohen \& Irani, 2018). Matlab function imresize (I, scale), where the default method is bicubic interpolation with antialiasing, and scale is the downsampling factor input to the function.

Table 2 comprises a list of datasets frequently used in SR and information on total image count, image format, pixel count, HR resolution, type of dataset, and classes of images. scale factors using bicubic interpolation with antialiasing. Other than the mentioned datasets in Table 2, datasets like General-100 (Dong, Loy \& Tang, 2016), L20 (Timofte, Rothe \& Gool, 2016), and ImageNet (Deng et al., 2009) are also used in computer vision tasks. In recent times, researchers have preferred the use of multiple datasets for training/evaluation and testing the SR models; for instance, in (Bashir \& Ghouri, 2014; Lai et al., 2017; Sajjadi, Scholkopf \& Hirsch, 2017; Tong et al., 2017), the researchers used SET5, SET14, BSDS100, and URBAN100 for training and testing.

\subsection{Super-resolution Challenges}

341

The most prominent SR challenges NTIRE (Agustsson \& Timofte, 2017), (Timofte et al., 2017), and PIRM (Blau et al., 2018), are discussed in this section. The New Trends in Image Restoration and Enhancement (NTIRE) challenge (Agustsson \& Timofte, 2017; Timofte et al., 2017) was in collaboration with the Conference on Computer Vision and Pattern Recognition (CVPR). NTIRE includes various challenges like colorization, image denoising, and SR. In the case of SR, the DIV2K dataset (Agustsson \& Timofte, 2017) was used, which included bicubic downscaled image pairs and blind images with realistic but unknown degradation. This dataset has been widely used to evaluate SR methods under known and unknown conditions to compare against the state-of-the-art methods. 
350

351

352

353

354

355

356

357

358

359

360

361

362

363

364

365

366

367

368

369

370

371

372

373

374

375

376

377

378

379

380

381

382

383

384

385

386

387

388

The perceptual image restoration and manipulation (PRIM) challenges were in collaboration with the European Conference on Computer vision (ECCV), and like NTIRE, it contained multiple challenges. Apart from the three challenges mentioned in NTIRE, PRIM also focused on SR for smartphones and compared perceptual quality with generation accuracy (Blau et al., 2018). As mentioned by (Blau \& Michaeli, 2018), the models that focus on distortion often give visually unpleasant SR images, while the models focusing on the perceptual image quality do not perform well on information fidelity. Using the image quality metrics NIQE (Mittal, Soundararajan \& Bovik, 2013) and (Ma et al., 2017b), the methods that performed best in achieving perceptual quality (Blau \& Michaeli, 2018) was the winner. In contrast, in a subchallenge (Ignatov et al., 2018b), SR methods were evaluated using limited resources to evaluate SR performance for smartphones using the PSNR, MS-SSIM, and opinion scoring metrics. Thus, PIRM encouraged the researchers to explore the perception-distortion tradeoff domain and SR for smartphones.

\section{Survey Methodology}

The majority of the studies included in this review paper are peer-reviewed publications to ensure the validity of the methods; these studies include conference proceedings and journal papers. The included papers include early access and a published version of recent papers for super-resolution from 2008 to 2021 . However, some in classical methods, some papers, and initial papers on image SR were included before this range to develop the review and give the background of the classical methods developed before the deep learning-based methods overtook the field. Google Scholar, IEEE Xplore, and Science Direct were queried to collect the initial list of papers in this research. Specific keywords were used to search the databases and based on the abstract. A further selection of papers was made using the reference sections of the selected papers as they contain additional relevant studies in image super-resolution. The last query was made on May 08, 2021. The collected papers were segregated based on their relevance with the Section; for example, papers with supervised learning were stored separately for review in Section 5, and studies highlighting the applications of SR methods were grouped for discussion in Section 7.

The relevant search terms include image super-resolution, super-resolution, deep learning superresolution, convolutional neural networks, image upsampling methods, super-resolution frameworks, supervised super-resolution, unsupervised super-resolution, super-resolution review, image interpolation, pixel-based methods, super-resolution application, assisted diagnosis using deep learning. The search keywords were not limited to single-image SR because our target was to report other aspects of super-resolution, including classical methods, applications, and datasets for SR.

Logical operators and wildcards were used to combine the keywords further and perform the additional search. Initial screening of the collected papers was performed following the inclusion/exclusion criteria shown in Table 3. The whole process is graphically shown in Fig. 3, 
389

390

391

392

393

394

395

396

397

398

399

400

401

402

403

404

405

406

407

408

409

410

411

412

413

414

415

416

417

418

419

420

421

422

423

424

425

426

427

where 653 studies were collected over one year. A total of 242 studies were included from the initial 653 collected research studies.

\section{Conventional Methods of Super-Resolution}

Classical methods of SR are briefly discussed in this section to encompass the overall development cycle of the SR. The classical methods include prediction-based, edge-based, statistical, patch-based, and sparse representation methods.

The primary methods were based on prediction, and the first method (Duchon, 1979) was based on Lanczos filtering, which filtered the digital data using sigma factors (with modifiable weight function), and a similar frequency-domain filtering approach was used in (Tsai \& Huang, 1984) for image resampling. In contrast, cubic convolution (Keys, 1981) was used for resampling the image data, and the results showed that this prediction method was more accurate than the nearest-neighbor prediction algorithm and linear interpolation of image data (Parker, Kenyon \& Troxel, 1983). In (Tsai \& Huang, 1984), the authors did not consider the blur in the imaging process, while (Irani \& Peleg, 1991) used the knowledge of the imaging process and the relative displacements for image interpolation when the sampling rate was kept constant and this method reduced to deblurring.

The patch-based approach was used in (Freeman, Jones \& Pasztor, 2002); the authors used a training set where various patches within the training set were extracted as training patterns, which helped generate detailed high-frequency images using the patch texture information. In (Chang, Yeung \& Xiong, 2004), the authors used locally linear embedding to use local patches for generating high-resolution images based on the local patch features. In contrast, (Glasner, Bagon \& Irani, 2009) used the concept of reoccurrence of geometrically similar patches in natural images to select the best possible pixel value based on the patch redundancy on the same scales. In (Baker \& Kanade, 2002), the authors introduced the concept of hallucination, where they extracted local features within the LR image first and used these to map the HR image. Edge-based methods use edge smoothness priors to upsample images, and in (Sun, Xu \& Shum, 2008), a generic image prior, gradient prior profile was used to smoothen the edges within an image to achieve super-resolution in natural images. In (Freedman \& Fattal, 2011), the authors used specially designed filters to search for similar patches using the local self-similarity observation, which performed lower nearest patch computations; this method was able to reconstruct realistic-looking edges, whereas it performed poorly in clustered regions with fine details.

Statistical methods were used to perform image super-resolution (Kim \& Kwon, 2010), where the authors used Kernel ridge regression (KRR) with gradient descent to learn the mapping function from the image example pairs. Adaptive regularization was used to supervise the energy change during the image resampling iterative process. This provided more accurate results as the energy map was used to limit the energy change per iteration, which reduced the noise while maintaining the perceptual quality (Xiong, Sun \& Wu, 2010) while (Yang et al., 2010), (Yang et 
428 al., 2008) used sparse representation methods to perform image super-resolution which used the 429 concept of compressed sensing.

430 The robust SR method proposed in (Zomet, Rav-Acha \& Peleg, 2001) used the information of

431 outliers to improve the performance of SR in patches where other methods introduce noise due to 432 these outliers. Additionally, (Yang et al., 2007) proposed a post-processing model that enhanced 433 the resolution of a set of images using a single reference image up to 100x scaling factor. 434 Another way to achieve SR is to use LR images to achieve a single HR image (Tipping \& 435 Bishop, 2003). The conventional upsampling methods, such as interpolation-based, use the

436

437

438

439

440

441

442

443

444

445

446

447

448

449

450

451

452

453

454

455

456

457

458

459

460

461

462

463

464

465

466 information within the LR image to generate HR images, and these methods do not add any new information to the image (Farsiu et al., 2004a). Furthermore, they also introduce some inherent problems, such as noise amplification and blur enhancement. Thus, in recent years, the researchers have shifted to learning-based upsampling methods explored in Section 5.

\section{Supervised Super-resolution}

Various deep learning methods were developed over the years to solve the SR problem; in this section, the models discussed are trained using both low and high-resolution images (LR-HR pairs). Although there are significant differences in the supervised SR models, and the models can be classified based on the components like the upsampling method employed, deep learning network, learning algorithm, and model frameworks. Any supervised image SR model is based on the combinations of these components, and in this section, we summarize the employed methods for these four components in light of recent supervised image SR research studies. The component-based review of various methods is performed in this section, and the basic overview of the models is shown in Fig. 1.

\subsection{Upsampling Methods}

The upsampling is essential in deep learning-based SR methods such as its positioning, and the method performed for upsampling has a significant impact on the training and test performance of the model. There are some commonly used methods (Yang et al., 2010), (Yang et al., 2008), (Lee, Yang \& Oh, 2015; Timofte, De Smet \& Van Gool, 2015), which use the conventional CNNs for end-to-end learning. In this subsection, various deep learning-based upsampling layers are discussed.

As mentioned in Section 4, the interpolation-based methods of upsampling do not add any new information; hence, learning-based methods are used in image SR in the last decade.

\subsubsection{Sub-pixel Layer}

The end-to-end learning layer (Shi et al., 2016), called the sub-pixel layer, performs upsampling by generating several additional channels using convolution, and by reshaping these channels, this layer performs upsampling, as shown in Fig. 4. In this layer, convolution is applied to $s_{f}^{2}$ where $s_{f}$ is the scaling factor, as shown in Fig. 4b. Since the input image size is $h \times w \times c$ where 
467

468

469

470

471

472

473

474

475

476

477

478

479

480

481

482

483

484

485

486

487

488

489

490

491

492

493

494

495

496

497

498

499

500

501

502

503

504

505

$h$ is height, $w$ is width, and $c$ depicts color channels, the resulting convolution is $h \times w \times c s_{f}^{2}$. In order to achieve the final image, a reshuffling (Shi et al., 2016) operation is performed to get the final output image $s_{f} h \times s_{f} w \times c$, as shown in Fig. 4c. Since it is an end-to-end layer, this layer is frequently used in SR models (Ledig et al., 2017), (Zhang, Zuo \& Zhang, 2018), (Ahn, Kang \& Sohn, 2018a), (Zhang et al., 2018b).

This layer has a wide receptive field, which helps learn more contextual information that generates realistic details, whereas this layer may generate some false artifacts at the boundaries of complex patterns due to its uneven distribution of the respective field. Furthermore, predicting the neighborhood pixels in a block-type region sometimes results in unsmooth outputs that do not look realistic when compared with the true HR image; to address this issue, PixelTCL (Gao et al., 2020) was proposed that used the interdependent prediction layer, which used the information of the interlinked pixels during upsampling. The results were smooth and more realistic when compared with the ground truth image.

\subsubsection{Deconvolution Layer}

The deconvolution layer also referred to as transposed convolution layer (Zeiler et al., 2010), is the converse of the convolution, i.e., predicting the probable input HR-image based on the feature maps from the LR image. In this process, additional zeros are inserted to increase the resolution, and afterwards, convolution is performed. For instance, taking scaling factor 2 for the SR image, a convolution kernel of $3 \times 3$ (as shown in Fig. 5a, 5b, and 5c), the input LR image is expanded twice by inserting zeros, convolution with the kernel is performed by using a stride and padding of 1 .

The deconvolution layer is widely used in SR methods (Sroubek, Cristobal \& Flusser, 2008; Hugelier et al., 2016; Lam et al., 2017; Tong et al., 2017; Haris, Shakhnarovich \& Ukita, 2018), as it generates HR images in an end-to-end way, and it has compatibility with the vanilla convolution. As per (Odena, Dumoulin \& Olah, 2017), in some cases, this layer may cause the problem of uneven overlapping within the generated HR image as the patterns are replicated in a check-like format and may result in a non-realistic HR image, thereby decreasing the performance of the SR method.

\subsubsection{Meta Upscaling}

The scaling factor was predefined in the previously mentioned methods, thereby training multiple upsampling modules with different factors, which is often inefficient and is not the actual requirement of an SR method. A meta upscaling module (Hu et al., 2019) was proposed; this module uses arbitrary scaling factors to generate SR image-based in meta-learning. Meta scaling module projects every position in the required HR image to a small patch in the given LR feature maps $j \times j \times c_{i}$, where $\mathrm{j}$ is arbitrary, and ci is the total number of channels within the extracted feature map (in (Hu et al., 2019) this was 64). Additionally, it also generates the convolution weights $\left(j \times j \times\left(c_{i} \times c_{o}\right)\right)$, where $c_{o}$ represents the output image channels, and it is 
506 usually 3 . Thus, the meta upscaling module continuously uses arbitrary scaling factors within a 507 single model and using a substantial training set, a large number of factors are simultaneously

508 trained. The performance of this layer even surpasses the results produced with fixed factor

509 models, and even though this module predicts the weights during the inference time, the overall

510 execution time for weight prediction is 100 times less than the total time required for feature

511 extraction (Hu et al., 2019). In cases where there is a need for larger magnifications, this module

512 may become unstable as it predicts the convolution weights for every pixel independent of the

513 image information within those pixels.

514 This upscaling method is frequently used in recent years, particularly in post-upsampling

515 frameworks (Section 5.4.2). The high-level representations extracted from the low-level

516 information are used to construct an HR image using meta upscaling in the last layer of the

517 model, making this method an end-to-end SR approach.

518 The comparison of upsampling methods is shown in Table 4; most SR methods use

519 deconvolution or sub-pixel layers for upscaling. However, for multiple scale factors, meta

520 upscaling is used.

521

522

523

524

525

526

527

528

529

530

531

532

533

534

535

536

537

\subsection{Deep learning SR Networks}

The network design and advancements in design architecture are recent trends in deep learning, and in SR, researchers have tried several design implications along with the SR framework (as seen in Section 5.4) for designing the overall SR network. Some of the fundamental and recent network designs are discussed in this section.

\subsubsection{Recursive Learning}

One of the basic network-based learning strategies is to use the same module for recursively learning high-level features. This method also minimizes the parameters as the strategy is based on the same module being updated recursively, as shown in Fig. 6a.

One of the most used recursive networks is the Deeply-recursive Convolutional Network (DRCN) (Kim, Lee \& Lee, 2016b). Utilizing a single convolution layer DRCN reaches up to a $41 \times 41$ repetitive field without requiring additional parameters, which is very deep compared to the Super-resolution Convolution Neural Network SRCNN (Thapa et al., 2016) $(13 \times 13)$. The Deep Recursive Residual Network (DRRN) (Tai, Yang \& Liu, 2017) utilized a ResBlock (He et al., 2016) as part of the recursive module for a total of 25 recursions and was reported to achieve better performance than the baseline ResBlock. Using the concept of DRCN, (Tai et al., 2017) proposed a memory block-based method MemNet which contained six recursive ResBlocks. Whereas the Cascading Residual Network (CARN) (Ahn, Kang \& Sohn, 2018a) also used ResBlocks as recursive units. In this approach, the network shares the weights globally in 543 recursions, the researchers also used Dual-state Recurrent Network (DSRN) (Han et al., 2018), 544 which shared the signals between the LR and generated HR states within the network. 
545 Overall, while reducing the parameters, recursive learning networks can learn the complex 546 representation of the data at the cost of computational performance. Additionally, the increase in 547 computational requirements may result in an exploding or vanishing gradient. Thus, recursive

548 learning is often used in combination with multi-supervision or residual learning for minimizing 549 the risk of exploding or vanishing gradient (Kim, Lee \& Lee, 2016b; Tai et al., 2017; Tai, Yang 550 \& Liu, 2017; Han et al., 2018).

551

552

553

554

555

556

557

558

559

560

561

562

563

564

565

566

567

568

569

570

571

572

573

574

\subsubsection{Residual Learning}

Residual learning was widely used in the field of SR (Bevilacqua et al., 2012), (Timofte, De Smet \& Van Gool, 2015), (Timofte, De \& Gool, 2013), until ResNet (He et al., 2016) was proposed for learning residuals, as shown in Fig. 6b. Overall, there are two approaches, local and global residual learning.

The local residual learning approach mitigates the degradation problem (He et al., 2016) caused by increased network depth. Furthermore, the local residual learning also improved the learning rate and reduced the training difficulty; this is frequently used in the SR field (Protter et al., 2009; Mao, Shen \& Yang, 2016; Han et al., 2018; Li et al., 2018).

The global residual learning is an approach used in which the input and the final output are correlated, and in image SR, the output HR is highly correlated with the input LR image; thus, learning the global residuals between LR and HR image is significant in SR. In global residual learning, the model only learns the residual map that transforms the LR image into an HR image by generating the missing high-frequency details in the LR image. Furthermore, the residuals are minimal; thereby, the learning difficulty and model complexity are significantly reduced in global residual-based learning. This method is also frequently used in SR methods (Kim, Lee \& Lee, 2016a), (Tai, Yang \& Liu, 2017), (Tai et al., 2017), (Hui, Wang \& Gao, 2018).

Overall, both methods use residuals to connect the input image with the output HR image; in the case of global residual learning, the connection is directly made, which in local residual learning various layers of different depth to connect the input (using local residuals) with the output.

\subsubsection{Dense Connection-based Learning}

575 dense block utilizes all the features maps generated by the previous layers as inputs and its 576 feature inputs, leading to $l(l-1) / 2$ connections in an 1-layer $(l \geq 2)$ dense block. Using dense

577 blocks will increase the reusability of the features while resolving the gradient vanishing 578 problem. Furthermore, the dense connections also minimize the model size by utilizing a small

579

580 growth rate and enfolding the channels using concatenated input features.

581 Dense connections are used in SR to connect the low-level and high-level features maps for reconstructing a high-quality fine-detailed HR image, as shown in Fig. 6c. SRDenseNet (Tong et al., 2017) proposed a 69-layer network containing dense connections within the dense blocks and dense connections among the dense blocks. In SRDenseNet, the feature maps from the prior blocks and the feature maps were used as inputs of all preceding blocks. RDN (Zhang et al., 
585

586

587

588

589

590

591

592

593

594

595

596

597

598

599

600

601

602

603

604

605

606

607

608

609

610

611

612

613

614

615

616

617

618

619

620

621

622

623

2018b), CARN (Ahn, Kang \& Sohn, 2018a), MemNet (Tai et al., 2017), and ESRGAN (Wang et al., 2019c) also used layer or block-level dense connection, while DBPN (Wang et al., 2018b) only used the dense connection between the upsampling and downsampling units.

\subsubsection{Multi-path Learning}

In multi-path learning, the features are transferred to multiple paths for different representations, and these representations are later combined to gain improved performance. Scale-specific, local, and global multi-path learnings are the main types.

For different scales, the super-resolution models use different feature extraction; in (Lim et al., 2017), the authors proposed a single network-based multi-path learning for multiple scales. The intermediate layers of the model were shared for feature extraction, while scale-specific paths, including pre-processing and upsampling, were at the end of the models, i.e., the start and end of the network. During training, the scale relative paths are enabled and updated accordingly, and the proposed deep super-resolution MDSR method (Lim et al., 2017) also decreases the overall model size because of the sharing of parameters across the scales. Like MDSR, a similar multipath-based approach is also implemented in ProSR and CARN.

Local multi-path learning is inspired using a new block, the inception module (Szegedy et al., 2015), for multi-scale feature extraction, as performed in MSRN (Li et al., 2018) (shown in Fig. $6 \mathrm{~d})$. The additional block consists of $3 \times 3$ and $5 \times 5$ kernel size convolution layers, which simultaneously extracts the features. After combining the outputs of the two convolution layers, the final output goes through a $5 \times 5$ kernel convolution. Furthermore, a path links the input and output by element-wise addition and uses this local multi-path learning; this method extracts features efficiently than multi-scale learning.

Another variation of multi-path learning is global multi-path learning; in this method, various features are extracted from multi-paths that can interact. In DSRN (Han et al., 2018), there are two paths for extracting low and high-level information, and there is a continuous sharing of features for improved learning. In contrast, in pixel recursive SR (Dahl, Norouzi \& Shlens, 2017), a conditioning path is responsible for extracting global structures, and the prior path further finds the serial codependence among the generated pixels. A different method was employed by (Ren, El-Khamy \& Lee, 2017), where multi-path learning was performed for unbalanced structures, which were later combined in the final layer to get the SR output.

\subsubsection{Advanced Convolution-based Learning}

In SR, the methods explored depend on the convolution operation, and various research studies have attempted to modify the convolution operation for better performance. In recent years, research studies have shown that group convolution, as shown in Fig. 6e, decreased the total number of parameters at the cost of small loops in performance (Hui, Wang \& Gao, 2018), (Johnson, Alahi \& Fei-Fei, 2016). In CARN-M (Ahn, Kang \& Sohn, 2018a) and IDN (Hui, Wang \& Gao, 2018), group convolution was used instead of vanilla convolution. In dilated convolution, the contextual information is used to generate realistic-looking SR images (Zhang 
624 et al., 2017); dilated convolution was used to double the receptive field, resulting in better 625 results.

626 Another type of convolution is depthwise separable convolution (Howard et al., 2009); although

627 this convolution significantly reduces the total number of parameters, it reduces the overall

628 performance.

629

630

\subsubsection{Attention-based Learning}

631 In deep learning, attention learning is the idea where certain factors are given more preference, 632 which processes the data than others; here, two types of attention-based learning mechanisms are 633 discussed in SR. In channel attention, a particular block is added in the model where global 634 average pooling (GAP) squeezes the input channels; two fully connected layers process these 635 constants to generate channel-wise residuals (Hu, Shen \& Sun, 2018), as shown in Fig. 6f. This technique has been incorporated in SR, known as RCAN (Zhang et al., 2018a), which has

638 improved performance. Instead of GAP, (Dai et al., 2019) used the second-order channel

639 attention (SPCA) module, which used second-order feature metric for extracting more data representation using channel-based attention

640 In SR, most of the models use local fields for the generation of SR pixels, while in a few cases,

641

642

643

644

645

646

647

648

649

650

651

652

653

654

655

656

657

658

659

660

661

662

663 some textures or patches which are far apart are necessary for generating accurate local patches. In (Zhang et al., 2019b), local and non-local attention blocks were used to extract local and nonlocal representations between pixel data. Similarly, the non-local attention technique was incorporated by (Dai et al., 2019) to capture contextual information using a non-local attention method. Chen et al. proposed an SR reconstruction method with feature maps to facilitate the reconstruction of the image using an attention mechanism (Chen et al., 2021), while Yang et al. proposed a channel attention and spatial graph convolutional network (CASGCN) for a more robust feature obtaining and feature correlations modeling (Yang \& Qi, 2021).

\subsubsection{Wavelet transform-based Learning}

Wavelet transform (WT) (Daubechies \& Bates, 1993; Griffel \& Daubechies, 1995) represents textures using high-frequency sub-bands and global structural information in low-frequency subbands in a highly efficient way. WT was used in SR to generate the residuals of the HR subbands using the sub-bands of the interpolated LR wavelet. Using the WT, the LR image is decomposed, while the inverse WT provides the reconstruction of the HR image in SR. Other examples of WT based SR are Wavelet-based residual attention network (WRAN) (Xue et al., 2020), multi-level wavelet CNN (MWCNN) (Liu et al., 2018b), and (Ma et al., 2019); these approaches used a hybrid approach by combining WT with other learning methods to improve the overall performance.

\subsubsection{Region-recursive-based Learning}

In SR, most methods follow the underlying assumption that it is a pixel-independent process; thus, there is no priority to the interdependence among the generated pixels. Using the concept of 
664 PixelCNN (Van Den Oord et al., 2016), (Dahl, Norouzi \& Shlens, 2017) proposed a method for 665 pixel recursive learning, which performed SR by pixel-by-pixel generation using two networks.

666 The two networks (Dahl, Norouzi \& Shlens, 2017) captured information about pixel dependence 667 and global contextual information within the pixel recursive SR method. Using the mean opinion 668 scoring-based evaluation method (Dahl, Norouzi \& Shlens, 2017) performed well compared to 669 other methods for generating SR face images using the pixel recursive method. The attention670 based face hallucination method (Cao et al., 2017a) also utilized the concept of a path-based 671 attention shifting mechanism to enhance the details in the local patches.

672 While the region-recursive methods perform marginally better than other methods, the recursive 673 process exponentially increases the training difficulty and computation costs due to long 674 propagation paths.

675

676

677

\subsubsection{Other Methods}

678

679

680

681

682

683

684

685

686

687

688

689

690

691

692

693

694

695

696

697

698

699

700

701

702

703

Other SR networks are also used by researchers, such as Desubpixel-based learning (Vu et al., 2019), xUnit-based learning (Kligvasser, Shaham \& Michaeli, 2018) and Pyramid Pooling-based learning (Zhao et al., 2017).

To improve the computational speed, the desubpixel-based approach was used to extract features in a low-dimensional space, which does the inverse task of the sub-pixel layer. By segmenting the images spatially and using them as separate channels, the desubpixel-based learning avoids any information loss; after learning the data representations in low-dimensional space, the images are upsampled to get a high-resolution image. This technique is particularly efficient in applications with limited resources such as smartphones.

In xUnit learning, a spatial activation function was proposed for learning complicated features and textures. In xUnit, the ReLU operation was replaced by xUnit to generate the weight maps through Gaussian gating and convolution. The model size was decreased by $50 \%$ using xUnit at the cost of increased computational demand without compromising the SR performance (Kligvasser, Shaham \& Michaeli, 2018).

\subsection{Learning Strategies}

Learning strategies also dictate the overall performance of any SR algorithm as the evaluations are dependent upon the choice of the learning strategy selected. In this section, recent research studies are discussed using the learning strategy utilized in SR, and some of the critical strategies are discussed in detail.

\subsubsection{Loss Functions}

For any application in deep learning, the selection of the loss functions is critical, and in SR, these functions are used to measure the error in the reconstruction of HR, which further helps optimize the model iteratively. Since the necessary element of the images is a pixel, initial research studies employed the pixel loss, L2, but it was evaluated that the pixel loss cannot wholly represent the quality of reconstruction (Ghodrati et al., 2019). Thus, in SR, different loss 
704 functions such as content loss (Johnson, Alahi \& Fei-Fei, 2016) or adversarial loss (Ledig et al., 705 2017) are used to measure the error in the generation these loss functions have been widely used 706 in the field of SR. Various loss functions are explored in this section, and the notation follows 707 the previously defined variables except where defined otherwise.

708 Content Loss. The perceptual quality, as mentioned previously, is essential in the evaluation of 709 an SR model, and this loss was used in SR (Johnson, Alahi \& Fei-Fei, 2016), (Dosovitskiy \& 710 Brox, 2016) to measure the differences between the generated and ground-truth images using an 711 image classification network $(\mathrm{N})$. Let the high-level data representation on the $l^{\text {th }}$ lth layer is $712 r^{l}(I)$, the content loss is defined as the Euclidean among the high-level representations of the

713 two images $I$ and $\hat{I}$, where $I$ is the original image and $\hat{I}$ is the generated SR image as below:

714

$$
\mathrm{L}\left(I, \hat{I} ; N_{c}, l\right)=\frac{1}{h_{l} w_{l} c_{l}} \sqrt{\sum_{i, j, k}\left(r_{i, j, k}^{l}(\hat{I})-r_{i, j, k}^{l}(I)\right)^{2}}
$$

715 Where $h_{l}, w_{l}$, and $c_{l}$ respectively are height, width, and several channels of the image

716 representations in the $l$ layer.

717 Content loss aims to share information about image features from the image classification

718 network $N_{c}$ to the SR network. This loss function ensures the visual similarity between the

719 original image $(I)$ and the generated image $(\hat{I})$ by comparing the content and not the individual 720 pixels. Thus, this loss function helps in producing visually perceptible and more realistic looking 721 images in the field of SR as in (Ledig et al., 2017), (Wang et al., 2018b), (Sajjadi, Scholkopf \& 722 Hirsch, 2017), (Wang et al., 2019c), (Johnson, Alahi \& Fei-Fei, 2016), (Bulat \& Tzimiropoulos, 723 2018) where the networks used as pre-trained CNNs were ResNet (He et al., 2016) and VGG 724 (Simonyan \& Zisserman, 2015).

725 Adversarial Loss. In recent years, after the development of GANs (Goodfellow et al., 2014), 726 GANs have received more consideration due to their ability to learn and self-supervise. A GAN 727 combines dual networks performing generation and discrimination tasks, i.e., generating the 728 actual output and using a discriminator network to evaluate the results of the generative network. 729 While training the GANs, two continuous updates were performed, i.e. (i) Adjust the generator 730 for better results while training the discriminator to discriminate more efficiently and (ii) Adjust 731 the discriminator while training the generator. This is a recursive training network, and through 732 many iterations of training and evaluation, the generator can generate the output that conforms to 733 the distribution of the actual data. The discriminator is unable to differentiate between real and 734 generated information.

735 In terms of image SR, the purpose of a generative network is to generate an HR image, while 736 another discriminator network will be used to evaluate if the image is of the same distribution as 737 the input data. This method was first introduced in SR as SRGAN (Ledig et al., 2017), the 738 adversarial loss in (Ledig et al., 2017) was represented by:

$$
\mathrm{L}_{G A N_{-} C E_{-} g}(\hat{I} ; D)=-\log D(\hat{I})
$$


740

$$
\mathrm{L}_{\text {GAN_CE_d }}\left(\hat{I}, I_{s} ; D\right)=-\left\{\log D\left(I_{s}\right)+\log (1-D(\hat{I}))\right\}
$$

741 Where $\mathrm{L}_{G A N_{-} C E_{-} g}$. is the adversarial loss function of the generator in the SR model, while

$742 \mathrm{~L}_{G A N_{-} C E_{-} d}$ is the adversarial loss function of the discriminator $D$, which is a binary classifier.

743 In (17), the randomly sampled ground truth image is denoted by $I_{s}$. The same loss functions

744 were reported by (Sajjadi, Scholkopf \& Hirsch, 2017).

745 Other than binary classification error, the studies (Yuan et al., 2018) and (Wang et al., 2018a)

746 used mean square error for improved training and better results compared to (Ledig et al., 2017),

747 the loss functions are given in (18) and (19):

748

749

$$
\mathrm{L}_{G A N_{-} L S \_g}(\hat{I} ; D)=(D(\hat{I})-1)^{2}
$$

$$
\mathrm{L}_{\text {GAN_LS_d }}\left(\hat{I}, I_{s} ; D\right)=\left\{(D(\hat{I}))^{2}+\left(D\left(I_{s}\right)-1\right)^{2}\right\}
$$

750

751

752

753

754

755

756

757

758

759

760

761

762

763

764

765

766

767

768

769

770

771

Contrary to the loss functions mentioned in (18) and (19), (Park et al., 2018) showed that in some cases, pixel-level discriminator network generates high-frequency noise; thus, we used another discriminator network to evaluate the first discriminator network for high-frequency representations. Using the two discriminator networks, (Park et al., 2018) were able to capture all attributes accurately.

Various opinion scoring systems have been used regressively to test the performance of the SR model that uses adversarial loss. Although the SR models attained lower PSNR than the pixelloss-based SR on perceptual quality metrics like opinion scoring, these adversarial loss-based SR methods scored very high (Ledig et al., 2017), (Sajjadi, Scholkopf \& Hirsch, 2017). The use of a discriminator as the control network for the generator GANs was able to regenerate some intricate patterns that were very difficult to learn using ordinary deep learning methods. The only drawback of the GANs is their training stability (Arjovsky, Chintala \& Bottou, 2017; Gulrajani et al., 2017; Lee et al., 2018a; Miyato et al., 2018).

Pixel Loss. As evident from the name, this loss function performs a pixel-wise comparison between the reference image and the generated image, and there are two types of comparisons, i.e., an $L 1$ loss, which is also termed as mean absolute error and $L 2$ loss, which is the mean square error (MSE)

$$
\begin{gathered}
\mathrm{L}_{P I X_{-} L 1}(I, \hat{I})=\frac{1}{h w c} \sum_{i, j, k}\left|I_{i, j, k}-\hat{I}_{i, j, k}\right| \\
\mathrm{L}_{P I X_{-} L 2}(I, \hat{I})=\frac{1}{h w c_{i, j, k}} \sum_{i, j, k}-\left.\hat{I}_{i, j, k}\right|^{2}
\end{gathered}
$$

The $L 1$ loss in some cases becomes numerically unstable to compute; thus, another variant of the L1 loss called the Charbonnier loss (Farsiu et al., 2004b; Barron, 2017, 2019; Lai et al., 2017) is given by: 


$$
\mathrm{L}_{P I X_{-} C H}(I, \hat{I})=\frac{1}{h w c} \sum_{i, j, k} \sqrt{\left|I_{i, j, k}-\hat{I}_{i, j, k}\right|^{2}-e^{2}}
$$

773 Here $e$ is a constant which ensures numerical stability.

774 The pixel loss function ensures that the generated HR image $\hat{I}$ has the same pixel values as the

775

776

777

778

779

780

781

782

783

784

785

786

787

788

789

790

791

792

793

794

795

796

797

798

799

800

801

802

803

804

HR image $I$. Furthermore, the L2 loss used the square of pixel-value errors, giving more weightage to high-value differences than lower ones; thus, this loss function may give either the too variable result (in case of outliers) or give too smooth results (in case of minimal error values). Therefore, the L1 loss function is widely used over L2 loss (Zhao et al., 2016; Lim et al., 2017; Ahn, Kang \& Sohn, 2018a). Furthermore, the PSNR equation is closely related to the definition of L1 loss, and minimizing L1 loss always leads to increased PSNR. Thus, researchers have often used the L1 loss to maximize the PSNR; as mentioned earlier, the pixel loss function does not cater to perceptual quality or textures. Thus, SR networks based on this loss function may have less high-frequency details, resulting in smooth but unrealistic HR images (Wang, Simoncelli \& Bovik, 2003; Wang et al., 2004).

Style Reconstruction Loss. Ideally, the reconstructed HR image should have comparable styles to the actual HR image (colors, textures, gradient, contrast), thus using the research studies (Sajjadi, Scholkopf \& Hirsch, 2017), (Gatys, Ecker \& Bethge, 2015), style reconstruction loss was used in SR to match the texture details of the reference image with the generated image. The correlation between the feature maps of different channels as given by the Gram matrix (Levy \& Goldberg, 2014) $G^{(l)} . G_{i, j}^{(l)}$ is the dot product of the features $i$, and $j$ in the layer $l$, it is which is given by:

$$
G_{i, j}^{(l)}=\operatorname{vec}\left(\operatorname{ch}_{i}^{(l)}(I)\right) \cdot \operatorname{vec}\left(\operatorname{ch}_{j}^{(l)}(I)\right)
$$

Where vec( ) is the vectorization operation and $c h_{i}^{(l)}$ denoted the $i^{\text {th }}$ channel of feature maps in the layer $l$. Now the texture loss is given by (24)

$$
\mathrm{L}_{T E X}(I, \hat{I} ; c h, l)=\frac{1}{c_{l}^{2}} \sqrt{\sum_{i, j}\left(G_{i, j}^{(l)}(I)-G_{i, j}^{(l)}(\hat{I})\right)^{2}}
$$

Using the texture loss function in (24), EnhanceNet (Sajjadi, Scholkopf \& Hirsch, 2017) reported more realistic results that look visually similar to the reference HR image. Although an optimized texture loss function-based SR generates more realistic-looking images, the selection of patch size is still an open field of research. The selection of small patch size leads to the generation of artifacts in the textured region, while selecting a big patch size generates artifacts across the whole image as the patches are averages over the whole image.

Total Variation Loss. Using the pixel values of the neighboring pixels, the total variation loss (Rudin, Osher \& Fatemi, 1992) was defined as the sum of the absolute difference among the values of the neighboring pixels as:

$$
\mathrm{L}_{T V}(\hat{I})=\frac{1}{h w c} \sum_{i, j, k} \sqrt{\left(\hat{I}_{i+1, j, k}-\hat{I}_{i, j, k}\right)^{2}-\left(\hat{I}_{i, j+1, k}-\hat{I}_{i, j, k}\right)^{2}}
$$


805 Total variation loss was used in (Ledig et al., 2017; Yuan et al., 2018) to ensure smoothness

806 across sharp edges/transitions within the generated image.

807 Cycle Consistency Loss. Using the CycleGAN (Zhu et al., 2017a) image SR method was

808 presented in (Yuan et al., 2018) using the cyclic consistency loss function. Using the generated

809 HR image $\hat{I}$, the network generated another LR image $I_{L R}^{\prime}$, which is further compared with the

810 input LR image $I_{L R}$ for cyclic consistency.

811 In practice, various loss functions are used as a combination in SR to ensure various aspects of

812 the generation process in the form of a weighted average as in (Kim, Lee \& Lee, 2016a), (Wang

813 et al., 2018b), (Sajjadi, Scholkopf \& Hirsch, 2017), (Lai et al., 2017). The selection of

814 appropriate weights of the loss functions in itself is another learning problem as the results vary

815 significantly by varying the weights of the loss function in image SR.

816

817 5.3.2. Curriculum Learning

818 In the Curriculum learning technique (Bengio et al., 2009), the method adapts itself to the

819 variable difficulty of tasks, i.e., starting from simple images with minimum noise to complex

820 images. Since SR always suffers from adverse conditions, the curriculum approach is mainly

821 applied to its learning difficulty and network size. For reducing the training difficulty of the

822 network in SR, small scaling factor, SR is performed in the beginning; in the curriculum

823 learning-based SR, the training starts with $2 x$ upsampling, and gradually the following scaling

824 factors $4 \times, 8 \times$, and so on are generated using the output of previously trained networks. ProSR

825 (Wang et al., 2018a) uses the upsampled output of the previous level and linearly trains the next

826 level using the previous one, while ADRSR (Bei et al., 2018) concatenates the HR output of the

827 previous levels and further adds another convolution layer. In CARN (Ahn, Kang \& Sohn,

828 2018b), the previously generated image is entirely replaced by the next level generated image,

829 updating the HR image in sequential order.

830 Another alternative is to transform the image SR problem into N subsets and gradually solving

831 these problems; as in (Park, Kim \& Chun, 2018), the $8 \times$ upsampling problem was divided into

832 three problems (i.e., $1 \times$ to $2 \times ; 2 \times$ to $4 \times$ and $4 \times$ to $8 \times$ ) and three separate networks were used to

833 solve these problems. Using a combination of the previous reconstruction, the next level was

834 finetuned in this method. The same concept was used in ( $\mathrm{Li}$ et al., 2019b) to train the network

835 from low image degradations to high image degradations, thus gradually increasing the noise in

836 the LR input image. Curriculum learning reduces the training difficulty; hence, the total

837 computational time is also reduced.

838

839 5.3.3. Batch Normalization

840 Batch normalization (BN) was proposed by (Ioffe \& Szegedy, 2015) to stabilize and accelerate

841 the deep CNNs by reducing the internal covariate shift of the network. Every mini-batch was

842 normalized, and two additional parameters were used per channel to preserve the representation

843 ability. Batch normalization is responsible for working on the intermediate feature maps; thus, it

844 resolves the vanishing gradient issue while allowing high learning rates. This technique is widely 
845 used in SR models such as (Ledig et al., 2017), (Zhang, Zuo \& Zhang, 2018), (Tai, Yang \& Liu, 846 2017), (Tai et al., 2017), (Ledig et al., 2017; Sønderby et al., 2017; Tai et al., 2017; Tai, Yang \&

847 Liu, 2017; Liu et al., 2018b; Zhang, Zuo \& Zhang, 2018). In contrast, (Lim et al., 2017) claimed 848 that batch normalization-based networks lose the scale information of the generated images.

849 Thus, there is a lack of flexibility in the network; hence, (Lim et al., 2017) removed batch

850 normalization and used the additional memory to design a large model with superior

851 performance compared to the BN-based network. Other studies (Wang et al., 2019c), (Wang et

852 al., 2018a), (Chen et al., 2018a) also implemented this technique to achieve marginally better

853 performance.

854

855

856

\subsubsection{Multi-supervision}

Using numerous supervision signals within the same model for improving the gradient

857 propagation and evading the exploding/vanishing gradient problem is called multi-supervision.

858 In (Kim, Lee \& Lee, 2016b), multi-supervision is incorporated within the recursive units to

859 address the gradient problems. In SR, the multi-supervision learning technique is implemented

860 by catering to a few other factors in the loss function, which improves the back-propagation path

861 and reduces the training difficulty of the model.

862

863

864

\subsection{SR Frameworks}

865

SR being an ill-posed problem; thus, upsampling is critical in defining the performance of the SR

866 method. Based on learning strategies, upsampling methods, and network types, there are several frameworks for SR; here, four of them are discussed in detail, especially in light of the

867 upsampling method used within the framework, as shown in Fig. $7-10$.

868

869

\subsubsection{Pre-upsampling $S R$}

870

871 Learning the mapping functions for upsampling from an LR image directly to an HR image is done using this framework, where the LR image is upsampled in the beginning, and various

872 convolution layers are used to extract representations in an iterative way using deep neural

873 networks. Using this concept (Dong et al., 2014), (Dong et al., 2016) introduced the pre-

874 upsampling-based SR framework (SRCNN), as shown in Fig. 7. SRCNN was used to learn the

875 end-to-end mapping of LR-HR image conversion using CNNs. Using the classical methods of

876 upsampling as discussed in Section 4, the LR image is firstly converted to an HR image, and

877 then deep CNNs were used to learn the representations for mapping the HR image.

878 Since the pre-upsampling layer already performs the actual pixel conversion task, the network

879 needs to refine the results using CNNs; this results in reduced learning difficulty. Compared to

880 single-scale SR (Kim, Lee \& Lee, 2016a), which uses specific scales of input, these models can

881

882 handle any random size image for refinement and have similar performance. In recent years, many application-oriented research studies have used this framework (Kim, Lee \& Lee, 2016b),

883 (Shocher, Cohen \& Irani, 2018), (Tai, Yang \& Liu, 2017), (Tai et al., 2017), the differences in

884 these models are in the deep learning layers employed after the upsampling. The only drawback 
885 in this model is the use of a predefined classical method of pre-upsampling, which often results 886 in the introduction of image blur, noise amplification in the upsampled image, which later affects 887 the quality of the concluding HR image. Moreover, the dimensions of the image are increased at 888 the start of the method. Thus, the computational cost and memory requirements of this 889 framework are higher (Shi et al., 2016).

890

891

892

\subsubsection{Post-upsampling SR}

893

894

895

896

897

898

899

900

901

902

To minimize the memory requirements and increase computational efficiency, the postupsampling method was used in SR to utilize deep learning to learn the mapping functions in low-dimensional space. This concept was first used in SR by (Shi et al., 2016) and (Dong, Loy \& Tang, 2016), and the network diagram is shown in Fig. 8.

Due to low computational costs and the use of low-dimensional space for deep learning, this model has been widely used in SR because this reduces the complexity of the model (Ledig et al., 2017; Lim et al., 2017; Tong et al., 2017; Han et al., 2018).

\subsubsection{Iterative Up-and-down Sampling SR}

Since the LR-HR mapping is an ill-posed problem, efficient learning using the LR-HR image

903 pair using back-propagation (Irani \& Peleg, 1991) was used in SR (Timofte, Rothe \& Gool,

904 2016). The SR network is called the iterative up-down sampling SR, as shown in Fig. 9. This

905 model refines the image using recursive back-propagation, i.e., continuously measuring the error 906 and refining the model based on the reconstruction error. The DBPN method proposed in (Haris,

907 Shakhnarovich \& Ukita, 2018) used this concept to perform continuous upsampling and

908 downsampling, and the final image was constructed using the intermediate generations of the HR image.

909 Similarly, SRFBN (Li et al., 2019b) used this technique with densely connected layers for image 910 SR, while RBPN (Haris, Shakhnarovich \& Ukita, 2019) used recurrent back-propagation with 911 iterative up-down upsampling for video SR. This framework has shown significant improvement 912 over the other frameworks; still, the back-propagation modules and their appropriate use require

913 further exploration as this concept is recently introduced.

914

915 5.4.4. Progressive-upsampling SR

916 Since the post-upsampling framework uses a single layer at the end of upsampling and the 917 learning is fixed for scaling factors; thus, multi-scale SR will increase the computational cost of 918 the post-upsampling framework. Thus, using progressive upsampling within the framework to 919 gradually achieve the required scaling was proposed, as seen in Fig. 10. An example of this 920 framework is the LapSRN (Lai et al., 2017), which uses cascaded CNN-based modules 921 responsible for mapping a single scaling factor, and the output of one module acts as the input 922 LR image to the other module. This framework was also used in ProSR (Wang et al., 2018a) and 923 MS-LapSRN (Lai et al., 2017). 
924 This model achieves higher learning rates as the learning difficulty is less since the SR operation 925 is segregated into several small upscaling tasks, which is more straightforward for CNNs to

926 learn. Furthermore, this model has built-in support for multi-scale SR as the images are scaled 927 with various intermediate scaling factors. Training stability and convergence are the main issues 928 with this framework, and this requires further research.

929

930

\subsection{Other Improvements}

931 Apart from the four primary considerations in image SR, other factors have a significant effect

932 on the performance of a super-resolution method, and in this section, a few are discussed in light of recent research.

934

935

\subsubsection{Data Augmentation}

936

937 Data augmentation is a common technique in deep learning, and this concept is used to further enhance the performance of a deep learning model by generating more training data using the

938 same dataset. In the case of image super-resolution, some of the augmentation techniques are

939 flipping, cropping, angular rotation, skew, and color degradation (Timofte, Rothe \& Gool, 2016;

940

941 Lai et al., 2017; Lim et al., 2017; Tai, Yang \& Liu, 2017; Han et al., 2018). Recoloring the image using channel shuffling in the LR-HR image pair is also used as data augmentation in image SR (Bei et al., 2018).

\subsubsection{Enhanced Prediction}

This data augmentation method affects the output HR image as multiple LR images are

946 augmented using rotation and flipping functions (Timofte, Rothe \& Gool, 2016). These

947

948 augmented are fed to the model for reconstruction, the reconstructed outputs are inversely

949 transformed, and the final HR image is based on the mean (Timofte, Rothe \& Gool, 2016), (Wang et al., 2018a) or median (Shocher, Cohen \& Irani, 2018) pixel values of the corresponding augmented outputs.

951

952

\subsubsection{Network Fusion and Interpolation}

953

This technique used multiple models to predict the HR image, and each prediction acts as the

954 input to the following network, like in context-wise network fusion (CNF) (Ren, El-Khamy \&

955 Lee, 2017). The CNF was based on three individual SRCNNs, and this model achieved the 956 performance, which was compared with the state-of-the-art SR models (Ren, El-Khamy \& Lee,

958

959 2017).

960

961

962 In the SR network, network interpolation is a model that uses PSNR-based and GAN-based models for image SR to boost SR performance. Network interpolation strategy (Wang et al., 2019c), (Wang et al., 2019b) used a PSNR-based model for training. In contrast, a GAN-based model was used for fine-tuning while the parameters were interpolated to get the weights of interpolation, and their results had few artifacts and look realistic. 


\section{5.5.4. Multi-task Learning}

965 Multi-task learning is used for learning various problems and getting a generalized model for

966

967

968

969

970

971

972

973

974

975

976

977

978

979

980

981

982

983

984

985

986

987

988

989

990

991

992

993

994

995

996

997

998

999

1000

1001

1002 representations found in learning, for example, image segmentation, object detection, and facial recognition (Caruana, 1997; Collobert \& Weston, 2008). In the field of super-resolution, (Wang et al., 2018b) used semantic maps as input to the model and predicted the parameters of the affine transformation on the transitional feature maps. The SFT-GAN in (Wang et al., 2018b) generated more realistic and crisp-looking images with good visual details regarding the textured regions. While in DNSR (Bei et al., 2018), a denoising network was proposed to denoise the output generated by the SR network; thus, using this closed-loop system (Bei et al., 2018) was able to achieve good results. Like DNSR, (Yuan et al., 2018) proposed an unsupervised SR using the cycle-in-cycle GAN (CinCGAN) for denoising during the SR task. Using a multi-tasking framework may increase the computational difficulty, but the system's performance is also enhanced in terms of PSNR and perceptual quality indexes.

\subsection{State-of-the-art SR Methods}

The recent year has excelled in developing SR models, especially using supervised deep learning; thus, the models have excelled in achieving state-of-the-art performance. Previously various aspects of the SR models and their underlying components were discussed in light of their strengths and weaknesses. In recent times the use of multiple learning strategies is common, and most of the state-of-the-art methods have used a combination of these strategies.

The first innovation was using the dual-branched network (DBCN) (Gao, Zhang \& Mou, 2019) to increase the computational efficiency of the single-branched network by using a smaller number of convolutional layers for representation. Furthermore, in RCAN (Zhang et al., 2018a), attention-based learning was used combined with residual learning, L1 pixel loss function, and subpixel upsampling method to achieve the state-of-the-art results in image SR. Furthermore, various models and their reported results and some key factors are summarized in Table 5. In previous sections, we discussed various strategies and compared and contrasted them; while these are important, the performance of any SR algorithm in comparison to the computational cost and parameters is also vital. In Fig. 11, we have graphically shown the performance of SR methods using PSNR metrics compared to their size (represented as several parameters) and computational cost (measured by the number of Multi-Adds). The datasets used in measurements are Set14, B100, and Urban 100; the overall PSNR is the average score over the three datasets, while the scaling factor for these models was fixed to $2 \times$.

As evident in Fig. 11, the five best-performing methods on the selected datasets based on PSNR are WRAN (34.790dB), RCAN (34.540dB), SAN (34.480dB), Meta-RDN (34.400dB). and EDSR ( $34.330 \mathrm{~dB})$. The variation in the average PSNR reported by these methods only varies in the range of $0.46 \mathrm{~dB}$. There is a significant difference in the number of parameters reported by these five methods; WRAN reported only 2.710 million parameters while EDSR reported the highest parameters among the five methods, i.e., 40.74 million. WRAN and RCAN performed 
1003 well in terms of PSNR, the number of parameters, and computational cost, thereby making them

1004

1005

1006

1007

1008

1009

1010

1011

1012

1013

1014

1015

1016

1017

1018

1019

1020

1021

1022

1023

1024

1025

1026

1027

1028

1029

1030

1031

1032

1033

1034

1035

1036

1037

1038

1039

1040

1041

1042

one of the best methods for image super-resolution.

\section{Unsupervised Super-resolution}

In this section, the methods of unsupervised SR are discussed, which does not require LR-HR pairs. The limitation of the supervised learning methods is that the LR images are usually generated using known degradations. In supervised learning, the model learns the reverse transformation function of the degradation function to convert the LR image into the HR image. Thus, using the unsupervised model to upsample the LR images is a field of growing interest, where the model learns the real-world image degradation to achieve SR using the information of unpaired LR and HR images. A few of the unsupervised SR models are discussed in the subsections.

\subsection{Weakly-supervised Super-resolution}

The first method to address the use of known degradation in the model for the generation of LR images using weakly supervised deep learning, this method utilized the unpaired LR and HR images for training the model. Although this model still requires both LR and HR images, the associations are not defined. Thus, there are two possible approaches; the first one is to learn the degradation function first, which can generate the degraded LR images and train the model to generate the HR images. The other method is to employ degradation function learning and LRHR mapping cyclically, thus validating the results with each other (Ignatov et al., 2018a).

\subsubsection{Cyclic weakly-supervised SR}

Using the unpaired LR and HR images and referring them to two separated uncorrelated datasets, this method uses a cycle-in-cycle approach to predict the mapping function of these two datasets, i.e., from LR to HR and HR to LR images. This is a recursive process where the mapping functions generate images with equal distribution, and these images are fed to the second prediction cyclically.

Using the deep learning-based CycleGAN (Zhu et al., 2017a), a cycle-in-cycle SR framework was proposed in (Yuan et al., 2018); this framework used a total of four generators, while there were two discriminators; the two GANS learned the representation of degraded LR to LR and LR to HR mappings. In (Yuan et al., 2018), the first generator is a simple denoising element that generates similar scale denoised LR images; these denoised images act as input to the second generator to regenerate the HR image, which is further validated by the adversarial network, i.e., a discriminator. Thus, using different loss functions, the CycleGAN achieves image SR using weakly supervised learning.

Although this method has achieved comparable results, especially in very noisy images where classical degradation functions in supervised learning cannot be used, there is room for research to decrease the learning difficulty of the computational cost of this method. 
1043

1044

1045

1046

1047

1048

1049

1050

1051

1052

1053

1054

1055

1056

1057

1058

1059

1060

1061

1062

1063

1064

1065

1066

1067

1068

1069

1070

1071

1072

1073

1074

1075

1076

1077

1078

1079

1080

1081

\subsubsection{Learning the degradation function}

A similar concept to the cyclic SR, but the two networks, i.e., a degradation learning network and LR-HR mapping network, are independently trained. In (Bulat, Yang \& Tzimiropoulos, 2018), a two-staged method of image SR was proposed, where a GAN learns the representations of the HR to LR transformation while the second GAN is trained using the paired output of the first GAN to learn the mapping representations of the LR to HR transformation. This two-stage model outperformed the state-of-the-art in Fréchet Inception Distance (FID) (Heusel et al., 2017) with $10 \%$ failure cases. This method reported superior reconstruction of HR human facial features.

\subsection{Zero-shot Super-resolution}

Using the training concept at the time of the test, the zero-shot SR (ZSSR) (Shocher, Cohen \& Irani, 2018) uses a single image to train a deep learning network using image augmentation techniques to learn the degradation function. ZSSR was used (Michaeli \& Irani, 2013) to predict the degradation kernel, which was further used to generate scaled and augmented images. The final step was to train an SRCNN network to learn the representations of this dataset, and in this way, the ZSSR uses augmentation and input image data to achieve SR. This model outdid the state-of-the-art for non-bicubic, noisy, and blurred LR images by $1 \mathrm{~dB}$ in the case of estimated kernels and $2 \mathrm{~dB}$ for known kernels.

Since this model requires training for every input image at the test time, the overall inference time is substantial.

\subsection{Image Prior in SR}

The low-level details in any learning problem can be mapped using CNNs, thus using a randomly initialized CNN as an image prior (Ulyanov, Vedaldi \& Lempitsky, 2020) to perform SR. The network is not trained; instead, it uses a random vector $v$ as input to the model, and it generates the HR image $I_{y H R}$. This method aims to determine an image $\hat{I}_{y H R}$ that, when downsampled, returns an LR image that is similar to the input LR image $\hat{I}_{x H R}$. The model performed $2 \mathrm{~dB}$ the state-of-the-art methods but reported superior results than the conventional bicubic upsampling method by $1 \mathrm{~dB}$.

\section{Domain-Specific Applications of Super-Resolution}

In this section, various applications of SR grouped by the application domains are discussed.

\subsection{Face Image Super-resolution}

Face hallucination $(\mathrm{FH})$ is perhaps the ultimate target utility of the image SR for facerecognition-based tasks such as (Gunturk et al., 2003), (Taigman et al., 2014; Korshunova et al., 
1082 2017; Zhang et al., 2018c; Grm, Scheirer \& Štruc, 2020). The facial images contain facial1083 structured information; thus, using image priors in FH has been a common approach to achieve 1084 FH.

1085 Using techniques such as in CBN (Zhu et al., 2016), the generated HR images can be constrained 1086 to face-related features, forcing the model to output HR images containing facial features. In 1087 CBN, this was achieved by using a facial prior and a dense correspondence field estimation. 1088 While in FSRNet (Chen et al., 2018b) facial parsing maps and facial landmark heatmaps were 1089 used as priors to the learning network to achieve face image SR, SICNN (Zhang et al., 2018c) 1090 used a joint training approach to recover the real identity using a super-identity loss function.

1091 Super-FAN (Bulat \& Tzimiropoulos, 2018) approached FH using end-to-end learning with FAN 1092 to ensure the generated images are consistent with human facial features.

1093 Using implicit methods for solving the face misalignment problem is another way to approach $1094 \mathrm{FH}$; for instance, in (Yu \& Porikli, 2017), the spatial transformation is achieved using 1095 transformation networks (Jaderberg et al., 2015). Another method based on (Jaderberg et al., 1096 2015) is TDAE (Wang et al., 2019b), which uses a three-module approach for FH, using a D-E1097 D (decoder-encoder-decoder) model to achieve $\mathrm{FH}$; the first decoder performs denoising and 1098 upsampling while encoder downsamples the denoised image which is fed to the final decoder for 1099 FH. Another approach is to use HR exemplars from datasets to decompose the facial features of

1100

1101

1102

1103

1104

1105

1106

1107

1108

1109

1110

1111

1112

1113

1114

1115

1116

1117

1118

1119

1120

1121

an LR image and project the HR features into the exemplar dataset to achieve FH (Yang, Liu \& Yang, 2018). In (Song et al., 2018), an adversarial discriminative network was proposed for feature learning on both feature space and raw pixel space; this method performed well for heterogenous face recognition (HFR).

In other research studies, human perception of attention shifting (Najemnik \& Geisler, 2005) was used in Attention-FH (Cao et al., 2017a) to learn face patches for local enhancement of FH. In (Xu et al., 2017), a multi-class GAN network was proposed for FH, which composed of multiple generators and discriminators, while in (Yu \& Porikli, 2016), the authors adopted a network model analogous to SRGAN (Ledig et al., 2017). Using conditional GAN (Gauthier, 2014), the studies (Lee et al., 2018b; Yu et al., 2018) used additional facial features to achieve FH with predefined attributes. Gao et al. proposed an efficient multilayer locality-constrained matrix regression (MLCMR) framework for face super-resolution of highly degraded LR images (Gao et al., 2021).

\subsection{Real-world Image Super-resolution}

In real-world images, the sensors used to capture them already introduce degradations as the final RGB (8-bit) image is converted from the raw image (usually more than 14-bit or higher). Thus, using these images as a reference for SR is not optimal as the images have already been degraded (Wang, Chen \& Hoi, 2020). To approach this problem, research studies such as (Zhang et al., 2019a) and (Chen et al., 2019) have proposed methods for developing real-world image datasets. In (Zhang et al., 2019a), the SR-RAW dataset was developed by the authors, which contained raw-HR-LR(RGB) pairs generated using the optical zoom in cameras, while in (Chen

Peer] Comput. Sci. reviewing PDF | (CS-2021:02:58423:2:0:NEW 3 Jun 2021) 
1122 et al., 2019), image resolution and its relationship with the field of view (FoV) were explored by

1123 the authors to generate a real-world dataset called City 100.

1124

1125

7.3. Depth Map Super-resolution

1126

In the field of computer vision, problems like image segmentation (Zaitoun \& Aqel, 2015; Yu \&

1127 Koltun, 2016; Kirillov et al., 2019)and pose estimation (Wei et al., 2016; Cao et al., 2017b; Chen

1128 \& Ramanan, 2017) have been approached by using depth maps. Depth maps retain the distance

1129 information of the scene and the observer, although these depth maps are of low-resolution

1130 because of the hardware constraints of the modern camera systems. Thus, image SR is used in this regard to increase the resolution of the depth maps. Using multiple cameras to record the same scene and generate multiple HR images is the most suitable way of doing depth map SR. In (Hui, Loy \& Tang, 2016), the authors used two separate CNNs to downsample HR image concurrently and upsample the LR depth map; after the generation of RGB features from the downsampling CNN, these features were used to fine-tune the upsampling process of depth maps. While (Riegler, Rüther \& Bischof, 2016) used the energy minimization model (such as (Bashir \& Ghouri, 2014)) to guide the model for generating HR depth maps without the need for reference images.

\subsection{Remote Sensing and Satellite Imaging}

The use of SR in improving the resolution of remote sensing and satellite imaging has increased in the past years (Shermeyer \& Van Etten, 2019). In (Li et al., 2017b), the authors used the concept of multi-line cameras to utilize multiple LR images to generate a high-quality HR image from the ZY-3 (TLC) satellite image dataset. In (Zhu et al., 2017b; Benecki et al., 2018), the authors argued that the conventional methods of evaluation of the SR techniques are not valid for satellite imaging as the degradation functions and operation conditions of the satellite hardware are entirely in a different environment and thus (Benecki et al., 2018) proposed a new way for validation of SR methods for satellite image SR methods. An adaptive multi-scale detail enhancement (AMDE-SR) was proposed in (Zhu et al., 2018) to use the multi-scale SR method to generate high-detailed HR images with accurate textual and high-frequency information. GAN-based methods provide superior performance for remote sensing image SR; Liu et al. developed a novel cascaded conditional Wasserstein generative adversarial network (CCWGAN) to generate HR images for remote sensing (Liu et al., 2020a). Bashir et al. proposed a YOLOv3based small-object detection framework SRCGAN-RFA-YOLO (Bashir \& Wang, 2021b), where the authors used residual feature aggregation and cyclic GAN to improve the resolution of remote sensing images before performing object detection.

\subsection{Video Super-resolution}

1160 In video SR, multiple frames represent the same scene; thus, there is inter and intra-frame spatial dependency in the video, which includes the information of brightness, colors, and relative motion of objects. Using the optical flow-based method (Sun, Roth \& Black, 2010; Liao et al., 
1162 2015), Sun et al. and Liao et al. proposed a method to generate probable HR candidate images 1163 and ensemble these images using CNNs. Using the Druleas (Drulea \& Nedevschi, 2011) 1164 algorithm, CVSRnet (Kappeler et al., 2016) addressed the effect of motion by using CNNs for 1165 the images in successive frames to generate HR images.

1166 Apart from direct learning motion compensation, a trainable spatial transformer (Jaderberg et al., 1167 2015) was used in VESPCN (Caballero et al., 2017) to motion compensation mapping using data 1168 from successive frames for end-to-end mapping. Using a sub-pixel layer-based module, (Tao et 1169 al., 2017) achieved super-resolution and motion compensation simultaneously.

1170 Another approach is to use recurrent networks to indirectly grasp the spatial and temporal 1171 interdependency to address the motion compensation. In STCN (Guo \& Chao, 2017), the authors 1172 used a bidirectional LSTM (Graves, Fernández \& Schmidhuber, 2005) and deep CNNs to extract 1173 the temporal and spatial information from the video frames, while BRCN (Huang, Wang \& 1174 Wang, 2015) utilized RNNs, CNNs, and conditional CNNs respectively for temporal, spatial and 1175 temporal-spatial interdependency mapping. Using 3D convolution filters of small size to replace 1176 the large-sized filter, FSTRN (Li et al., 2019a) achieves state-of-the-art performance using deep 1177 CNNs, sustaining a low computational cost. A novel spatio-temporal matching network (STMN) 1178 for video SR was proposed, which worked on the wavelet transform to minimize the dependence 1179 on motion estimations (Zhu et al., 2021).

1180

1181

1182

1183

1184

1185

1186

1187

1188

1189

1190

1191

1192

1193

1194

1195

1196

1197

1198

1199

1200

1201

\subsection{SR for Medical Imaging}

Other fields also used the concept of image super-resolution to achieve high-resolution images, such as in (Mahapatra, Bozorgtabar \& Garnavi, 2019); the authors proposed the use of progressive GANs to enhance the image quality of magnetic resonance (MR) images. DeepResolve (Chaudhari et al., 2018) used image SR methods to generate thin-sliced knee MR images from the thick-sliced input images. Since the diffusion MRI has high image acquisition time and low resolution, Super-resolution Reconstruction Diffusion Tensor Imaging (SRR-DTI) reconstructed HR diffusion parameters from LR diffusion-weighted (DW) images (Van Steenkiste et al., 2016). (Hamaide et al., 2017) also used SRR-DTI to find the structural sex variances in the adult zebra finch brain.

Assisted diagnosis using super-resolution has been a recent trend; for instance, researchers used deep learning-based SR methods to assist the diagnosis of movement disorders like isolated dystonia (Bashir \& Wang, 2021a).

\subsection{Other Applications}

Other applications of SR include object detection (Li et al., 2017a; Tan, Yan \& Bare, 2018), stereo image SR (Duan \& Xiao, 2019; Guo, Chen \& Huang, 2019; Wang et al., 2019a), and super-resolution in optical microscopy (Qiao et al., 2021). Overall, SR plays a vital role in multidisciplines, from medical science, computer vision to satellite imaging and remote sensing.

\section{Discussion and Future Directions}


1202 This paper gives an overall review of literature for image super-resolution, and the contribution 1203 of this paper is discussed in this section.

1204

\subsection{Learning Strategies}

1206

Learning strategies in image SR are introduced in Section 5.3; while the learning strategies are

1207 well matured in image SR, there are research directions in the development of alternate loss functions and alternative of batch normalization There are various loss functions in SR, and the choice of SR depends upon the task, while it is still an open research area to find an optimal loss function that fits all SR frameworks. A combination of loss functions is currently used to optimize the learning process, and there are no standard criteria for the selection of loss function; thus, exploring various probable loss functions for super-resolution is a promising future direction. Batch normalization is a technique that performs well in computer vision tasks and reduces the overall runtime of the training, and enhances the performance; however, in SR batch normalization proved to be sub-optimal (Lim et al., 2017), (Wang et al., 2018a), (Chen et al., 2018a). In this regard, normalization techniques for super-resolution should be explored further.

\subsection{Network Design}

1220

1221

Network design strategies require further exploration in SR as the network design inherently dictates the overall performance of any SR method. Some of the key research areas are highlighted in this section As discussed in Section 5.1, current upsampling methods have significant drawbacks for the deconvolution layer and may produce checkerboard artifacts. In contrast, the sub-pixel layer is susceptible to the non-uniform distribution of receptive fields; the meta-scale method has stability issues, while the interpolation-based methods lack end-to-end learning. Thus, further research is required to explore upsampling methods that can be generic to SR models and can be applied to LR images with any scaling factors. models may be trained to give more attention to some image features than others like the human visual system does. Using a combination of low and high-level representations simultaneously to accelerate the SR process is another field in network design for fast and accurate reconstruction of the HR image. Exploring network architectures that can be implemented in practical applications since current methods use deep neural networks, which increases the performance of the SR at the expense of higher computational cost; thus, research in the development of network architecture that is minimal and provides optimal performance is another promising research direction.

\subsection{Evaluation Metrics}

The image quality metrics used in SR act as the benchmark score, while the two most commonly used metrics, PSNR and SSIM, help gauge the performance of SR, but these metrics introduce 
1242 inherent issues in the generated image. Using PSNR as an evaluation metric usually introduces

1243

1244

1245

1246

1247

1248

1249

1250

1251

1252

1253

1254

1255

1256

1257

1258

1259

1260

1261

1262

1263

1264

1265

1266

1267

1268

1269

1270

1271

1272

1273

1274

1275

1276

1277

1278

1279

1280

1281

1282

non-realistic smooth surfaces, while SSIM works with textures, structures, brightness, and contrast to imitate human perception. These metrics cannot completely grasp the perceptual quality of images (Ledig et al., 2017), (Sajjadi, Scholkopf \& Hirsch, 2017). Opinion scoring is a metric that ensures perceptual quality, but this metric is impractical for implementing SR methods for large datasets; thus, a probable research direction is developing a universal quality metric for SR.

\subsection{Unsupervised Super-resolution}

In the past two years, unsupervised SR methods have gained popularity, but still, the task of collecting various resolution scenes for a similar pose is difficult; thus, bicubic interpolation is used instead to generate an unpaired SR dataset. In actuality, the unsupervised SR methods learn the inverse mapping of this interpolation for the reconstruction of HR images, and the actual learning of SR is still an open research field using unsupervised learning methods.

\section{Conclusion}

A detailed survey of classical SR and recent advances in SR with deep learning are explored in this survey paper. The central theme of this survey was to discuss deep learning-based SR techniques and the application of SR in various fields. Although image SR has achieved a lot in the last decade, some open problems are highlighted in Section 8. This survey is intended for the researchers in the field of SR and researchers from other fields to use image SR in their respective fields of interest.

\section{Acknowledgment}

We show our gratitude to the authors of all referred research studies for sharing results, especially to the authors of (Kim, Lee \& Lee, 2016b), (Ledig et al., 2017), (Dong, Loy \& Tang, 2016), (Lai et al., 2017), (Haris, Shakhnarovich \& Ukita, 2018), (Hu et al., 2019), (Tai, Yang \& Liu, 2017), (Tai et al., 2017), (Li et al., 2018), (Lim et al., 2017), (Zhang et al., 2018a), (Dai et al., 2019), (Xue et al., 2020), (Caballero et al., 2017).

\section{References}

Agustsson E, Timofte R. 2017. NTIRE 2017 Challenge on Single Image Super-Resolution: Dataset and Study. In: IEEE Computer Society Conference on Computer Vision and Pattern Recognition Workshops. 126-135. DOI: 10.1109/CVPRW.2017.150.

Ahn N, Kang B, Sohn KA. 2018a. Fast, accurate, and lightweight super-resolution with cascading residual network. In: Lecture Notes in Computer Science (including subseries Lecture Notes in Artificial Intelligence and Lecture Notes in Bioinformatics). 252-268. DOI: 10.1007/978-3-030-01249-6_16.

Ahn N, Kang B, Sohn KA. 2018b. Image super-resolution via progressive cascading residual network. In: IEEE Computer Society Conference on Computer Vision and Pattern Recognition Workshops. 791-799. DOI: 10.1109/CVPRW.2018.00123.

Peer] Comput. Sci. reviewing PDF | (CS-2021:02:58423:2:0:NEW 3 Jun 2021) 
1283

1284

1285

1286

1287

1288

1289

1290

1291

1292

1293

1294

1295

1296

1297

1298

1299

1300

1301

1302

1303

1304

1305

1306

1307

1308

1309

1310

1311

1312

1313

1314

1315

1316

1317

1318

1319

1320

1321

1322

1323

1324

1325

1326

1327

1328

Almohammad A, Ghinea G. 2010. Stego image quality and the reliability of PSNR. In: 2010 2nd International Conference on Image Processing Theory, Tools and Applications, IPTA 2010. 215-220. DOI: 10.1109/IPTA.2010.5586786.

Arjovsky M, Chintala S, Bottou L. 2017. Wasserstein generative adversarial networks. In: 34 th International Conference on Machine Learning, ICML 2017.

Baker S, Kanade T. 2002. Limits on super-resolution and how to break them. IEEE Transactions on Pattern Analysis and Machine Intelligence 24:1167-1183. DOI:

10.1109/TPAMI.2002.1033210.

Barron JT. 2017. A More General Robust Loss Function.

Barron JT. 2019. A general and adaptive robust loss function. In: Proceedings of the IEEE Computer Society Conference on Computer Vision and Pattern Recognition. 4331-4339. DOI: 10.1109/CVPR.2019.00446.

Bashir SMA, Ghouri FAK. 2014. Perspective texture synthesis based on improved energy optimization. PLoS ONE 9. DOI: 10.1371/journal.pone.0110622.

Bashir SMA, Wang Y. 2021a. Deep learning for the assisted diagnosis of movement disorders, including isolated dystonia. Frontiers in Neurology 12:638266. DOI: 10.3389 /fneur.2021.638266.

Bashir SMA, Wang Y. 2021b. Small Object Detection in Remote Sensing Images with Residual Feature Aggregation-Based Super-Resolution and Object Detector Network. Remote Sensing 13:1854. DOI: 10.3390/rs13091854.

Bates M, Huang B, Dempsey GT, Zhuang X. 2007. Multicolor super-resolution imaging with photo-switchable fluorescent probes. Science 317:1749-1753. DOI: $10.1126 /$ science. 1146598 .

Bei Y, Damian A, Hu S, Menon S, Ravi N, Rudin C. 2018. New techniques for preserving global structure and denoising with low information loss in single-image super-resolution. In: IEEE Computer Society Conference on Computer Vision and Pattern Recognition Workshops. 874-881. DOI: 10.1109/CVPRW.2018.00132.

Benecki P, Kawulok M, Kostrzewa D, Skonieczny L. 2018. Evaluating super-resolution reconstruction of satellite images. Acta Astronautica 153:15-25. DOI: 10.1016/j. actaastro.2018.07.035.

Bengio Y, Louradour J, Collobert R, Weston J. 2009. Curriculum learning. In: Proceedings of the 26th International Conference On Machine Learning, ICML 2009. 41-48.

Bevilacqua M, Roumy A, Guillemot C, Morel MLA. 2012. Low-complexity single-image superresolution based on nonnegative neighbor embedding. In: BMVC 2012 - Electronic Proceedings of the British Machine Vision Conference 2012. DOI: 10.5244/C.26.135.

Blau Y, Mechrez R, Timofte R, Michaeli T, Zelnik-Manor L. 2018. The 2018 PIRM challenge on perceptual image super-resolution. In: Proceedings of the European Conference on Computer Vision (ECCV). DOI: 10.1007/978-3-030-11021-5_21.

Blau Y, Michaeli T. 2018. The Perception-Distortion Tradeoff. In: Proceedings of the IEEE Computer Society Conference on Computer Vision and Pattern Recognition. 6228-6237. DOI: 10.1109/CVPR.2018.00652.

Borman S, Stevenson RL. 1998. Super-resolution from image sequences-A review. In: Midwest Symposium on Circuits and Systems. 374-378. DOI: 10.1109/MWSCAS.1998.759509.

Bosse S, Maniry D, Müller KR, Wiegand T, Samek W. 2018. Deep Neural Networks for NoReference and Full-Reference Image Quality Assessment. IEEE Transactions on Image Processing 27:206-219. DOI: 10.1109/TIP.2017.2760518. 
1329 Bulat A, Tzimiropoulos G. 2018. Super-FAN: Integrated Facial Landmark Localization and

1330

1331

1332

1333

1334

1335

1336

1337

1338

1339

1340

1341

1342

1343

1344

1345

1346

1347

1348

1349

1350

1351

1352

1353

1354

1355

1356

1357

1358

1359

1360

1361

1362

1363

1364

1365

1366

1367

1368

1369

1370

1371

1372

1373

1374
Super-Resolution of Real-World Low Resolution Faces in Arbitrary Poses with GANs. In: Proceedings of the IEEE Computer Society Conference on Computer Vision and Pattern Recognition. 109-117. DOI: 10.1109/CVPR.2018.00019.

Bulat A, Yang J, Tzimiropoulos G. 2018. To learn image super-resolution, use a GAN to learn how to do image degradation first. In: Proceedings of the European conference on computer vision (ECCV). 185-200. DOI: 10.1007/978-3-030-01231-1_12.

Caballero J, Ledig C, Aitken A, Acosta A, Totz J, Wang Z, Shi W. 2017. Real-time video superresolution with spatio-temporal networks and motion compensation. In: Proceedings - 30th IEEE Conference on Computer Vision and Pattern Recognition, CVPR 2017. 4778-4787. DOI: $10.1109 / C V P R .2017 .304$.

Cai D, Chen K, Qian Y, Kämäräinen JK. 2019. Convolutional low-resolution fine-grained classification. Pattern Recognition Letters 119:166-171. DOI: 10.1016/j.patrec.2017.10.020.

Candès EJ, Fernandez-Granda C. 2014. Towards a mathematical theory of super-resolution. Communications on Pure and Applied Mathematics 67:906-956. DOI: 10.1002/cpa.21455.

Cao Q, Lin L, Shi Y, Liang X, Li G. 2017a. Attention-aware face hallucination via deep reinforcement learning. In: Proceedings - 30th IEEE Conference on Computer Vision and Pattern Recognition, CVPR 2017. 690-698. DOI: 10.1109/CVPR.2017.180.

Cao Z, Simon T, Wei SE, Sheikh Y. 2017b. Realtime multi-person 2D pose estimation using part affinity fields. In: Proceedings - 30th IEEE Conference on Computer Vision and Pattern Recognition, CVPR 2017. 7291-7299. DOI: 10.1109/CVPR.2017.143.

Caruana R. 1997. Multitask Learning. Machine Learning 28:41-75. DOI: 10.1023/A:1007379606734.

Chang H, Yeung DY, Xiong Y. 2004. Super-resolution through neighbor embedding. In: Proceedings of the IEEE Computer Society Conference on Computer Vision and Pattern Recognition. I-I. DOI: 10.1109/cvpr.2004.1315043.

Chaudhari AS, Fang Z, Kogan F, Wood J, Stevens KJ, Gibbons EK, Lee JH, Gold GE, Hargreaves BA. 2018. Super-Resolution Musculoskeletal MRI Using Deep Learning. Magnetic Resonance in Medicine 80:2139-2154. DOI: 10.1002/mrm.27178.

Chen Y, Liu L, Phonevilay V, Gu K, Xia R, Xie J, Zhang Q, Yang K. 2021. Image superresolution reconstruction based on feature map attention mechanism. Applied Intelligence:1-14. DOI: 10.1007/s10489-020-02116-1.

Chen R, Qu Y, Zeng K, Guo J, Li C, Xie Y. 2018a. Persistent memory residual network for single image super resolution. In: IEEE Computer Society Conference on Computer Vision and Pattern Recognition Workshops. 809-816. DOI: 10.1109/CVPRW.2018.00125.

Chen CH, Ramanan D. 2017. 3D human pose estimation $=2 \mathrm{D}$ pose estimation + matching. In: Proceedings - 30th IEEE Conference on Computer Vision and Pattern Recognition, CVPR 2017. 7035-7043. DOI: 10.1109/CVPR.2017.610.

Chen Y, Tai Y, Liu X, Shen C, Yang J. 2018b. FSRNet: End-to-End Learning Face SuperResolution with Facial Priors. In: Proceedings of the IEEE Computer Society Conference on Computer Vision and Pattern Recognition. DOI: 10.1109/CVPR.2018.00264.

Chen C, Xiong Z, Tian X, Zha ZJ, Wu F. 2019. Camera lens super-resolution. In: Proceedings of the IEEE Computer Society Conference on Computer Vision and Pattern Recognition. DOI: 10.1109/CVPR.2019.00175.

Collobert R, Weston J. 2008. A unified architecture for natural language processing: Deep neural 
1375

1376

1377

1378

1379

1380

1381

1382

1383

1384

1385

1386

1387

1388

1389

1390

1391

1392

1393

1394

1395

1396

1397

1398

1399

1400

1401

1402

1403

1404

1405

1406

1407

1408

1409

1410

1411

1412

1413

1414

1415

1416

1417

1418

1419

1420

networks with multitask learning. In: Proceedings of the 25th International Conference on Machine Learning. 160-167.

Dabov K, Foi A, Katkovnik V, Egiazarian K. 2006. Color image denoising via sparse 3D collaborative filtering with grouping constraint in luminance-chrominance space. In: Proceedings - International Conference on Image Processing, ICIP. I-313. DOI: 10.1109/ICIP.2007.4378954.

Dahl R, Norouzi M, Shlens J. 2017. Pixel Recursive Super Resolution. In: Proceedings of the IEEE International Conference on Computer Vision. 5439-5448. DOI: 10.1109/ICCV.2017.581.

Dai T, Cai J, Zhang Y, Xia ST, Zhang L. 2019. Second-order attention network for single image super-resolution. In: Proceedings of the IEEE Computer Society Conference on Computer Vision and Pattern Recognition. 11065-11074. DOI: 10.1109/CVPR.2019.01132.

Daubechies I, Bates BJ. 1993. Ten Lectures on Wavelets. The Journal of the Acoustical Society of America. DOI: 10.1121/1.406784.

Deng X. 2018. Enhancing Image Quality via Style Transfer for Single Image Super-Resolution. IEEE Signal Processing Letters 25:571-575. DOI: 10.1109/LSP.2018.2805809.

Deng J, Dong W, Socher R, Li L-J, Kai Li, Li Fei-Fei. 2009. ImageNet: A large-scale hierarchical image database. In: 2009 IEEE conference on computer vision and pattern recognition. 248-255. DOI: 10.1109/cvpr.2009.5206848.

Dong C, Loy CC, He K, Tang X. 2014. Learning a deep convolutional network for image superresolution. In: Lecture Notes in Computer Science (including subseries Lecture Notes in Artificial Intelligence and Lecture Notes in Bioinformatics). DOI: 10.1007/978-3-31910593-2_13.

Dong C, Loy CC, He K, Tang X. 2016. Image Super-Resolution Using Deep Convolutional Networks. IEEE Transactions on Pattern Analysis and Machine Intelligence 38:295-307. DOI: 10.1109/TPAMI.2015.2439281.

Dong C, Loy CC, Tang X. 2016. Accelerating the super-resolution convolutional neural network. In: Lecture Notes in Computer Science (including subseries Lecture Notes in Artificial Intelligence and Lecture Notes in Bioinformatics). 391-407. DOI: 10.1007/978-3-31946475-6 25.

Dosovitskiy A, Brox T. 2016. Generating images with perceptual similarity metrics based on deep networks. In: Advances in Neural Information Processing Systems. 658-666.

Drulea M, Nedevschi S. 2011. Total variation regularization of local-global optical flow. In: IEEE Conference on Intelligent Transportation Systems, Proceedings, ITSC. 318-323. DOI: 10.1109/ITSC.2011.6082986.

Duan C, Xiao N. 2019. Parallax-Based Spatial and Channel Attention for Stereo Image SuperResolution. IEEE Access 7:183672-183679. DOI: 10.1109/ACCESS.2019.2960561.

Duchon CE. 1979. Lanczos filtering in one and two dimensions. Journal of applied meteorology 18:1016-1022. DOI: 10.1175/1520-0450(1979)018<1016:LFIOAT>2.0.CO;2.

Farsiu S, Robinson D, Elad M, Milanfar P. 2004a. Advances and challenges in super-resolution. International Journal of Imaging Systems and Technology 14:47-57. DOI: 10.1002/ima.20007.

Farsiu S, Robinson MD, Elad M, Milanfar P. 2004b. Fast and robust multiframe super resolution. IEEE Transactions on Image Processing 13:1327-1344. DOI: 10.1109/TIP.2004.834669.

Fernández-Suárez M, Ting AY. 2008. Fluorescent probes for super-resolution imaging in living cells. Nature Reviews Molecular Cell Biology 9:929-943. DOI: 10.1038/nrm2531. 
1421 Freedman G, Fattal R. 2011. Image and video upscaling from local self-examples. $A C M$

1422

1423

1424

1425

1426

1427

1428

1429

1430

1431

1432

1433

1434

1435

1436

1437

1438

1439

1440

1441

1442

1443

1444

1445

1446

1447

1448

1449

1450

1451

1452

1453

1454

1455

1456

1457

1458

1459

1460

1461

1462

1463

1464

1465

1466 Transactions on Graphics 30:1-11. DOI: 10.1145/1944846.1944852.

Freeman WT, Jones TR, Pasztor EC. 2002. Example-based super-resolution. IEEE Computer Graphics and Applications 22:56-65. DOI: 10.1109/38.988747.

Gao G, Yu Y, Xie J, Yang J, Yang M, Zhang J. 2021. Constructing multilayer localityconstrained matrix regression framework for noise robust face super-resolution. Pattern Recognition 110:107539. DOI: 10.1016/j.patcog.2020.107539.

Gao H, Yuan H, Wang Z, Ji S. 2020. Pixel Transposed Convolutional Networks. IEEE Transactions on Pattern Analysis and Machine Intelligence 1:1-1. DOI: 10.1109/TPAMI.2019.2893965.

Gao X, Zhang L, Mou X. 2019. Single Image Super-Resolution Using Dual-Branch Convolutional Neural Network. IEEE Access 7:15767-15778. DOI: 10.1109/ACCESS.2018.2889760.

Gatys LA, Ecker AS, Bethge M. 2015. Texture synthesis using convolutional neural networks. In: Advances in Neural Information Processing Systems. 262-270.

Gauthier J. 2014. Conditional generative adversarial nets for convolutional face generation. Technical report.

Gerchberg RW. 1974. Super-resolution through error energy reduction. Optica Acta 21:709-720. DOI: $10.1080 / 713818946$.

Ghodrati V, Shao J, Bydder M, Zhou Z, Yin W, Nguyen KL, Yang Y, Hu P. 2019. MR image reconstruction using deep learning: Evaluation of network structure and loss functions. Quantitative Imaging in Medicine and Surgery 9:1516. DOI: 10.21037/qims.2019.08.10.

Glasner D, Bagon S, Irani M. 2009. Super-resolution from a single image. In: Proceedings of the IEEE International Conference on Computer Vision. 349-356. DOI: 10.1109/ICCV.2009.5459271.

Goodfellow IJ, Pouget-Abadie J, Mirza M, Xu B, Warde-Farley D, Ozair S, Courville A, Bengio Y. 2014. Generative adversarial nets. In: Advances in Neural Information Processing Systems. 2672-2680.

Goyal M, Lather Y, Lather V. 2015. Analytical Relation \& Comparison of PSNR and SSIM on Baboon Image and Human Eye Perception Using MATLAB. International Journal of Advanced Research in Engineering and Applied Sciences 4:108-119.

Graves A, Fernández S, Schmidhuber J. 2005. Bidirectional LSTM networks for improved phoneme classification and recognition. In: Lecture Notes in Computer Science (including subseries Lecture Notes in Artificial Intelligence and Lecture Notes in Bioinformatics). 799-804.

Griffel DH, Daubechies I. 1995. Ten Lectures on Wavelets. The Mathematical Gazette 79:224227. DOI: $10.2307 / 3620105$.

Grm K, Scheirer WJ, Štruc V. 2020. Face Hallucination Using Cascaded Super-Resolution and Identity Priors. IEEE Transactions on Image Processing 29:2150-2165. DOI: 10.1109/TIP.2019.2945835.

Gulrajani I, Ahmed F, Arjovsky M, Dumoulin V, Courville A. 2017. Improved training of wasserstein GANs. In: Advances in Neural Information Processing Systems. 5767-5777.

Gunturk BK, Batur AU, Altunbasak Y, Hayes MH, Mersereau RM. 2003. Eigenface-domain super-resolution for face recognition. IEEE Transactions on Image Processing 12:597-606. DOI: 10.1109/TIP.2003.811513.

Guo J, Chao H. 2017. Building an end-to-end spatial-temporal convolutional network for video

PeerJ Comput. Sci. reviewing PDF | (CS-2021:02:58423:2:0:NEW 3 Jun 2021) 
1467

1468

1469

1470

1471

1472

1473

1474

1475

1476

1477

1478

1479

1480

1481

1482

1483

1484

1485

1486

1487

1488

1489

1490

1491

1492

1493

1494

1495

1496

1497

1498

1499

1500

1501

1502

1503

1504

1505

1506

1507

1508

1509

1510

1511

1512 super-resolution. In: 31st AAAI Conference on Artificial Intelligence, AAAI 2017.

Guo C, Chen D, Huang Z. 2019. Learning Efficient Stereo Matching Network with Depth Discontinuity Aware Super-Resolution. IEEE Access 7:159712-159723. DOI: 10.1109/ACCESS.2019.2950924.

Ha VK, Ren JC, Xu XY, Zhao S, Xie G, Masero V, Hussain A. 2019. Deep Learning Based Single Image Super-resolution: A Survey. International Journal of Automation and Computing 16:413-426. DOI: 10.1007/s11633-019-1183-x.

Hamaide J, De Groof G, Van Steenkiste G, Jeurissen B, Van Ruijssevelt L, Verhoye M, Van der Linden A, Cornil C, Sijbers J. 2017. Exploring sex differences in the adult zebra finch brain: In vivo diffusion tensor imaging and ex vivo super-resolution track density imaging. NeuroImage 146:789-803. DOI: 10.1016/j.neuroimage.2016.09.067.

Han W, Chang S, Liu D, Yu M, Witbrock M, Huang TS. 2018. Image Super-Resolution via Dual-State Recurrent Networks. In: Proceedings of the IEEE Computer Society Conference on Computer Vision and Pattern Recognition. 1654-1663. DOI:

10.1109/CVPR.2018.00178.

Haris M, Shakhnarovich G, Ukita N. 2018. Deep Back-Projection Networks for SuperResolution. In: Proceedings of the IEEE Computer Society Conference on Computer Vision and Pattern Recognition. 1664-1673. DOI: 10.1109/CVPR.2018.00179.

Haris M, Shakhnarovich G, Ukita N. 2019. Recurrent back-projection network for video superresolution. In: Proceedings of the IEEE Computer Society Conference on Computer Vision and Pattern Recognition. 3897-3906. DOI: 10.1109/CVPR.2019.00402.

He K, Zhang X, Ren S, Sun J. 2016. Deep residual learning for image recognition. In: Proceedings of the IEEE Computer Society Conference on Computer Vision and Pattern Recognition. 770-778. DOI: 10.1109/CVPR.2016.90.

Heusel M, Ramsauer H, Unterthiner T, Nessler B, Hochreiter S. 2017. GANs trained by a two time-scale update rule converge to a local Nash equilibrium. In: Advances in Neural Information Processing Systems. 6626-6637.

Horé A, Ziou D. 2010. Image quality metrics: PSNR vs. SSIM. In: Proceedings - International Conference on Pattern Recognition. 2366-2369. DOI: 10.1109/ICPR.2010.579.

Howard AG, Zhu M, Chen B, Kalenichenko D, Wang W, Weyand T, Marco A, Adam H. 2009. MobileNets: Efficient Convolutional Neural Networks for Mobile Vision Applications. In: Computer Vision and Pattern Recognition. DOI: 10.1016/S1507-1367(10)60022-3.

Hu X, Mu H, Zhang X, Wang Z, Tan T, Sun J. 2019. Meta-SR: A magnification-arbitrary network for super-resolution. In: Proceedings of the IEEE Computer Society Conference on Computer Vision and Pattern Recognition. 1575-1584. DOI: 10.1109/CVPR.2019.00167.

Hu J, Shen L, Sun G. 2018. Squeeze-and-Excitation Networks. In: Proceedings of the IEEE Computer Society Conference on Computer Vision and Pattern Recognition. 7132-7141. DOI: 10.1109/CVPR.2018.00745.

Huang G, Liu Z, Van Der Maaten L, Weinberger KQ. 2017. Densely connected convolutional networks. In: Proceedings - 30th IEEE Conference on Computer Vision and Pattern Recognition, CVPR 2017. 4700-4708. DOI: 10.1109/CVPR.2017.243.

Huang B, Wang W, Bates M, Zhuang X. 2008. Three-dimensional super-resolution imaging by stochastic optical reconstruction microscopy. Science 319:810-813. DOI: $10.1126 /$ science. 1153529.

Huang Y, Wang W, Wang L. 2015. Bidirectional recurrent convolutional networks for multiframe super-resolution. In: Advances in Neural Information Processing Systems. 235-243.

PeerJ Comput. Sci. reviewing PDF | (CS-2021:02:58423:2:0:NEW 3 Jun 2021) 
1513 Hugelier S, De Rooi JJ, Bernex R, Duwé S, Devos O, Sliwa M, Dedecker P, Eilers PHC,

1514

1515

1516

1517

1518

1519

1520

1521

1522

1523

1524

1525

1526

1527

1528

1529

1530

1531

1532

1533

1534

1535

1536

1537

1538

1539

1540

1541

1542

1543

1544

1545

1546

1547

1548

1549

1550

1551

1552

1553

1554

1555

1556

1557

1558
Ruckebusch C. 2016. Sparse deconvolution of high-density super-resolution images. Scientific Reports 6:21413. DOI: 10.1038/srep21413.

Hui TW, Loy CC, Tang X. 2016. Depth map super-resolution by deep multi-scale guidance. In: Lecture Notes in Computer Science (including subseries Lecture Notes in Artificial Intelligence and Lecture Notes in Bioinformatics). 353-369. DOI: 10.1007/978-3-31946487-9 22.

Hui Z, Wang X, Gao X. 2018. Fast and Accurate Single Image Super-Resolution via Information Distillation Network. In: Proceedings of the IEEE Computer Society Conference on Computer Vision and Pattern Recognition. 723-731. DOI: 10.1109/CVPR.2018.00082.

Ignatov A, Kobyshev N, Timofte R, Vanhoey K, Van Gool L. 2018a. WESPE: Weakly supervised photo enhancer for digital cameras. In: IEEE Computer Society Conference on Computer Vision and Pattern Recognition Workshops. 691-700. DOI:

10.1109/CVPRW.2018.00112.

Ignatov A, Timofte R, Van Vu T, Luu TM, Pham TX, Van Nguyen C, Kim Y, Choi JS, Kim M, Huang J, Ran J, Xing C, Zhou X, Zhu P, Geng M, Li Y, Agustsson E, Gu S, Van Gool L, de Stoutz E, Kobyshev N, Nie K, Zhao Y, Li G, Tong T, Gao Q, Hanwen L, Michelini PN, Dan Z, Fengshuo H, Hui Z, Wang X, Deng L, Meng R, Qin J, Shi Y, Wen W, Lin L, Feng R, Wu S, Dong C, Qiao Y, Vasu S, Thekke Madam N, Kandula P, Rajagopalan AN, Liu J, Jung C. 2018b. PIRM challenge on perceptual image enhancement on smartphones: Report. In: Proceedings of the European Conference on Computer Vision (ECCV). DOI: 10.1007/978-3-030-11021-5 20.

Ioffe S, Szegedy C. 2015. Batch normalization: Accelerating deep network training by reducing internal covariate shift. In: 32nd International Conference on Machine Learning, ICML 2015.

Irani M, Peleg S. 1991. Improving resolution by image registration. CVGIP: Graphical Models and Image Processing 53:231-239. DOI: 10.1016/1049-9652(91)90045-L.

Jaderberg M, Simonyan K, Zisserman A, Kavukcuoglu K. 2015. Spatial transformer networks. In: Advances in Neural Information Processing Systems. 2017-2025.

Johnson J, Alahi A, Fei-Fei L. 2016. Perceptual losses for real-time style transfer and superresolution. In: Lecture Notes in Computer Science (including subseries Lecture Notes in Artificial Intelligence and Lecture Notes in Bioinformatics). 694-711. DOI: 10.1007/978-3319-46475-6_43.

Jurek J, Kociński M, Materka A, Elgalal M, Majos A. 2020. CNN-based superresolution reconstruction of 3D MR images using thick-slice scans. Biocybernetics and Biomedical Engineering 40:111-125. DOI: 10.1016/j.bbe.2019.10.003.

Kappeler A, Yoo S, Dai Q, Katsaggelos AK. 2016. Super-resolution of compressed videos using convolutional neural networks. In: Proceedings - International Conference on Image Processing, ICIP. 1150-1154. DOI: 10.1109/ICIP.2016.7532538.

Kawulok M, Benecki P, Piechaczek S, Hrynczenko K, Kostrzewa D, Nalepa J. 2020. Deep Learning for Multiple-Image Super-Resolution. IEEE Geoscience and Remote Sensing Letters 17:1062-1066. DOI: 10.1109/LGRS.2019.2940483.

Keys RG. 1981. Cubic Convolution Interpolation for Digital Image Processing. IEEE Transactions on Acoustics, Speech, and Signal Processing 29:1153-1160. DOI: 10.1109/TASSP.1981.1163711.

Kim KI, Kwon Y. 2010. Single-image super-resolution using sparse regression and natural 
1559

1560

1561

1562

1563

1564

1565

1566

1567

1568

1569

1570

1571

1572

1573

1574

1575

1576

1577

1578

1579

1580

1581

1582

1583

1584

1585

1586

1587

1588

1589

1590

1591

1592

1593

1594

1595

1596

1597

1598

1599

1600

1601

1602

1603

1604

image prior. IEEE Transactions on Pattern Analysis and Machine Intelligence 32:11271133. DOI: 10.1109/TPAMI.2010.25.

Kim J, Lee S. 2017. Deep learning of human visual sensitivity in image quality assessment framework. In: Proceedings - 30th IEEE Conference on Computer Vision and Pattern Recognition, CVPR 2017. 1676-1684. DOI: 10.1109/CVPR.2017.213.

Kim J, Lee JK, Lee KM. 2016a. Accurate image super-resolution using very deep convolutional networks. In: Proceedings of the IEEE Computer Society Conference on Computer Vision and Pattern Recognition. 1646-1654. DOI: 10.1109/CVPR.2016.182.

Kim J, Lee JK, Lee KM. 2016b. Deeply-recursive convolutional network for image superresolution. In: Proceedings of the IEEE Computer Society Conference on Computer Vision and Pattern Recognition. 1637-1645. DOI: 10.1109/CVPR.2016.181.

Kirillov A, He K, Girshick R, Rother C, Dollar P. 2019. Panoptic segmentation. In: Proceedings of the IEEE Computer Society Conference on Computer Vision and Pattern Recognition. 9404-9413. DOI: 10.1109/CVPR.2019.00963.

Kligvasser I, Shaham TR, Michaeli T. 2018. XUnit: Learning a Spatial Activation Function for Efficient Image Restoration. In: Proceedings of the IEEE Computer Society Conference on Computer Vision and Pattern Recognition. 2433-2442. DOI: 10.1109/CVPR.2018.00258.

Korshunova I, Shi W, Dambre J, Theis L. 2017. Fast Face-Swap Using Convolutional Neural Networks. In: Proceedings of the IEEE International Conference on Computer Vision. 3677-3685. DOI: 10.1109/ICCV.2017.397.

Krizhevsky A, Sutskever I, Hinton GE. 2012. ImageNet classification with deep convolutional neural networks. In: Advances in neural information processing systems. 1097-1105. DOI: $10.1145 / 3065386$.

Lai WS, Huang J Bin, Ahuja N, Yang MH. 2017. Deep laplacian pyramid networks for fast and accurate super-resolution. In: Proceedings - 30th IEEE Conference on Computer Vision and Pattern Recognition, CVPR 2017. 624-632. DOI: 10.1109/CVPR.2017.618.

Lam F, Cladière D, Guillaume C, Wassmann K, Bolte S. 2017. Super-resolution for everybody: An image processing workflow to obtain high-resolution images with a standard confocal microscope. Methods 115:17-27. DOI: 10.1016/j.ymeth.2016.11.003.

Ledig C, Theis L, Huszár F, Caballero J, Cunningham A, Acosta A, Aitken A, Tejani A, Totz J, Wang Z, Shi W. 2017. Photo-realistic single image super-resolution using a generative adversarial network. In: Proceedings - 30th IEEE Conference on Computer Vision and Pattern Recognition, CVPR 2017. 4681-4690. DOI: 10.1109/CVPR.2017.19.

Lee S, Kim JH, Heo JP. 2020. Super-resolution of license plate images via character-based perceptual loss. In: Proceedings - 2020 IEEE International Conference on Big Data and Smart Computing, BigComp 2020. 560-563. DOI: 10.1109/BigComp48618.2020.000-1.

Lee K, Xu W, Fan F, Tu Z. 2018a. Wasserstein Introspective Neural Networks. In: Proceedings of the IEEE Computer Society Conference on Computer Vision and Pattern Recognition. 3702-3711. DOI: 10.1109/CVPR.2018.00390.

Lee D, Yang MH, Oh S. 2015. Fast and accurate head pose estimation via random projection forests. In: Proceedings of the IEEE International Conference on Computer Vision. 19581966. DOI: 10.1109/ICCV.2015.227.

Lee CH, Zhang K, Lee HC, Cheng CW, Hsu W. 2018b. Attribute augmented convolutional neural network for face hallucination. In: IEEE Computer Society Conference on Computer Vision and Pattern Recognition Workshops. 721-729. DOI: 10.1109/CVPRW.2018.00115. Levy O, Goldberg Y. 2014. Neural word embedding as implicit matrix factorization. In:

Peer] Comput. Sci. reviewing PDF | (CS-2021:02:58423:2:0:NEW 3 Jun 2021) 
1605

1606

1607

1608

1609

1610

1611

1612

1613

1614

1615

1616

1617

1618

1619

1620

1621

1622

1623

1624

1625

1626

1627

1628

1629

1630

1631

1632

1633

1634

1635

1636

1637

1638

1639

1640

1641

1642

1643

1644

1645

1646

1647

1648

1649

1650

Advances in Neural Information Processing Systems. 2177-2185.

Li C, Bovik AC. 2010. Content-partitioned structural similarity index for image quality assessment. Signal Processing: Image Communication 25:517-526. DOI: 10.1016/j.image.2010.03.004.

Li J, Fang F, Mei K, Zhang G. 2018. Multi-scale residual network for image super-resolution. In: Lecture Notes in Computer Science (including subseries Lecture Notes in Artificial Intelligence and Lecture Notes in Bioinformatics). 517-532. DOI: 10.1007/978-3-03001237-3_32.

Li S, He F, Du B, Zhang L, Xu Y, Tao D. 2019a. Fast spatio-temporal residual network for video super-resolution. In: Proceedings of the IEEE Computer Society Conference on Computer Vision and Pattern Recognition. 10522-10531. DOI: 10.1109/CVPR.2019.01077.

Li J, Liang X, Wei Y, Xu T, Feng J, Yan S. 2017a. Perceptual generative adversarial networks for small object detection. In: Proceedings - 30th IEEE Conference on Computer Vision and Pattern Recognition, CVPR 2017. 1222-1230. DOI: 10.1109/CVPR.2017.211.

Li L, Wang W, Luo H, Ying S. 2017b. Super-resolution reconstruction of high-resolution satellite ZY-3 TLC images. Sensors (Switzerland) 17:1062. DOI: 10.3390/s17051062.

Li X, Wu Y, Zhang W, Wang R, Hou F. 2020. Deep learning methods in real-time image superresolution: a survey. Journal of Real-Time Image Processing 17:1885-1909. DOI: 10.1007/s11554-019-00925-3.

Li Z, Yang J, Liu Z, Yang X, Jeon G, Wu W. 2019b. Feedback network for image superresolution. In: Proceedings of the IEEE Computer Society Conference on Computer Vision and Pattern Recognition. 3867-3876. DOI: 10.1109/CVPR.2019.00399.

Liao R, Tao X, Li R, Ma Z, Jia J. 2015. Video super-resolution via deep draft-ensemble learning. In: Proceedings of the IEEE International Conference on Computer Vision. 531-539. DOI: 10.1109/ICCV.2015.68.

Lim B, Son S, Kim H, Nah S, Lee KM. 2017. Enhanced Deep Residual Networks for Single Image Super-Resolution. In: IEEE Computer Society Conference on Computer Vision and Pattern Recognition Workshops. 136-144. DOI: 10.1109/CVPRW.2017.151.

Lin TY, Maire M, Belongie S, Hays J, Perona P, Ramanan D, Dollár P, Zitnick CL. 2014. Microsoft COCO: Common objects in context. In: European Conference on Computer Vision. 740-755. DOI: 10.1007/978-3-319-10602-1_48.

Liu B, Li H, Zhou Y, Peng Y, Elazab A, Wang C. 2020a. A super resolution method for remote sensing images based on cascaded conditional wasserstein GANs. In: $20203 \mathrm{rd}$ IEEE International Conference on Information Communication and Signal Processing, ICICSP 2020. 284-289. DOI: 10.1109/ICICSP50920.2020.9232066.

Liu Z, Luo P, Wang X, Tang X. 2015. Deep learning face attributes in the wild. In: Proceedings of the IEEE International Conference on Computer Vision. 3730-3738. DOI: 10.1109/ICCV.2015.425.

Liu H, Ruan Z, Zhao P, Shang F, Yang L, Liu Y. 2020b. Video super resolution based on deep learning: A comprehensive survey. arXiv.

Liu Di, Wang Z, Fan Y, Liu X, Wang Z, Chang S, Wang X, Huang TS. 2018a. Learning Temporal Dynamics for Video Super-Resolution: A Deep Learning Approach. IEEE Transactions on Image Processing 27:3432-3445. DOI: 10.1109/TIP.2018.2820807.

Liu X, Weijer J Van De, Bagdanov AD. 2017. RankIQA: Learning from Rankings for NoReference Image Quality Assessment. In: Proceedings of the IEEE International Conference on Computer Vision. 1040-104. DOI: 10.1109/ICCV.2017.118.

Peer] Comput. Sci. reviewing PDF | (CS-2021:02:58423:2:0:NEW 3 Jun 2021) 
1651

1652

1653

1654

1655

1656

1657

1658

1659

1660

1661

1662

1663

1664

1665

1666

1667

1668

1669

1670

1671

1672

1673

1674

1675

1676

1677

1678

1679

1680

1681

1682

1683

1684

1685

1686

1687

1688

1689

1690

1691

1692

1693

1694

1695

1696

Liu P, Zhang H, Zhang K, Lin L, Zuo W. 2018b. Multi-level wavelet-CNN for image restoration. In: IEEE Computer Society Conference on Computer Vision and Pattern Recognition Workshops. 773-782. DOI: 10.1109/CVPRW.2018.00121.

Ma K, Liu W, Liu T, Wang Z, Tao D. 2017a. DipIQ: Blind Image Quality Assessment by Learning-to-Rank Discriminable Image Pairs. IEEE Transactions on Image Processing 26:3951-3964. DOI: 10.1109/TIP.2017.2708503.

Ma K, Liu W, Zhang K, Duanmu Z, Wang Z, Zuo W. 2018. End-To-end blind image quality assessment using deep neural networks. IEEE Transactions on Image Processing 27:12021213. DOI: 10.1109/TIP.2017.2774045.

Ma W, Pan Z, Guo J, Lei B. 2019. Achieving Super-Resolution Remote Sensing Images via the Wavelet Transform Combined with the Recursive Res-Net. IEEE Transactions on Geoscience and Remote Sensing 57:3512-3527. DOI: 10.1109/TGRS.2018.2885506.

Ma C, Yang CY, Yang X, Yang MH. 2017b. Learning a no-reference quality metric for singleimage super-resolution. Computer Vision and Image Understanding 158:1-16. DOI: 10.1016/j.cviu.2016.12.009.

Mahapatra D, Bozorgtabar B, Garnavi R. 2019. Image super-resolution using progressive generative adversarial networks for medical image analysis. Computerized Medical Imaging and Graphics 71:30-39. DOI: 10.1016/j.compmedimag.2018.10.005.

Mao XJ, Shen C, Yang Y Bin. 2016. Image restoration using very deep convolutional encoderdecoder networks with symmetric skip connections. In: Advances in Neural Information Processing Systems. 2802-2810.

Martin D, Fowlkes C, Tal D, Malik J. 2001. A database of human segmented natural images and its application to evaluating segmentation algorithms and measuring ecological statistics. In: Proceedings of the IEEE International Conference on Computer Vision. 416-423. DOI: 10.1109/ICCV.2001.937655.

Michaeli T, Irani M. 2013. Nonparametric blind super-resolution. In: Proceedings of the IEEE International Conference on Computer Vision. 945-952. DOI: 10.1109/ICCV.2013.121.

Mittal A, Soundararajan R, Bovik AC. 2013. Making a "completely blind" image quality analyzer. IEEE Signal Processing Letters 20:209-212. DOI: 10.1109/LSP.2012.2227726.

Miyato T, Kataoka T, Koyama M, Yoshida Y. 2018. Spectral normalization for generative adversarial networks. In: 6th International Conference on Learning Representations, ICLR 2018 - Conference Track Proceedings.

Najemnik J, Geisler WS. 2005. Optimal eye movement strategies in visual search. Nature 434:387-391. DOI: 10.1038/nature03390.

Nasrollahi K, Moeslund TB. 2014. Super-resolution: A comprehensive survey. Machine Vision and Applications 25:1423-1468. DOI: 10.1007/s00138-014-0623-4.

Odena A, Dumoulin V, Olah C. 2017. Deconvolution and Checkerboard Artifacts. Distill 1:e3. DOI: $10.23915 /$ distill.00003.

Van Den Oord A, Kalchbrenner N, Vinyals O, Espeholt L, Graves A, Kavukcuoglu K. 2016. Conditional image generation with PixelCNN decoders. In: Advances in Neural Information Processing Systems. 4790-4798.

van Ouwerkerk JD. 2006. Image super-resolution survey. Image and Vision Computing 24:10391052. DOI: $10.1016 / \mathrm{j}$.imavis.2006.02.026.

Pambrun JF, Noumeir R. 2015. Limitations of the SSIM quality metric in the context of diagnostic imaging. In: Proceedings - International Conference on Image Processing, ICIP. 2960-2963. DOI: 10.1109/ICIP.2015.7351345. 
1697 Park D, Kim K, Chun SY. 2018. Efficient module based single image super resolution for

1698

1699

1700

1701

1702

1703

1704

1705

1706

1707

1708

1709

1710

1711

1712

1713

1714

1715

1716

1717

1718

1719

1720

1721

1722

1723

1724

1725

1726

1727

1728

1729

1730

1731

1732

1733

1734

1735

1736

1737

1738

1739

1740

1741

1742 multiple problems. In: IEEE Computer Society Conference on Computer Vision and Pattern Recognition Workshops. 882-890. DOI: 10.1109/CVPRW.2018.00133.

Park SC, Park MK, Kang MG. 2003. Super-resolution image reconstruction: A technical overview. IEEE Signal Processing Magazine 20:21-36. DOI: 10.1109/MSP.2003.1203207.

Park SJ, Son H, Cho S, Hong KS, Lee S. 2018. SRFeat: Single Image Super-Resolution with Feature Discrimination. In: Lecture Notes in Computer Science (including subseries Lecture Notes in Artificial Intelligence and Lecture Notes in Bioinformatics). 439-455. DOI: 10.1007/978-3-030-01270-0_27.

Parker JA, Kenyon R V., Troxel DE. 1983. Comparison of Interpolating Methods for Image Resampling. IEEE Transactions on Medical Imaging. DOI: 10.1109/TMI.1983.4307610.

Protter M, Elad M, Takeda H, Milanfar P. 2009. Generalizing the nonlocal-means to superresolution reconstruction. IEEE Transactions on Image Processing 18:36-51. DOI: 10.1109/TIP.2008.2008067.

Qiao C, Li D, Guo Y, Liu C, Jiang T, Dai Q, Li D. 2021. Evaluation and development of deep neural networks for image super-resolution in optical microscopy. Nature Methods 18:194202. DOI: $10.1038 / \mathrm{s} 41592-020-01048-5$.

Ravì D, Szczotka AB, Pereira SP, Vercauteren T. 2019. Adversarial training with cycle consistency for unsupervised super-resolution in endomicroscopy. Medical Image Analysis 53:123-131. DOI: 10.1016/j.media.2019.01.011.

Ravì D, Szczotka AB, Shakir DI, Pereira SP, Vercauteren T. 2018. Effective deep learning training for single-image super-resolution in endomicroscopy exploiting video-registrationbased reconstruction. International Journal of Computer Assisted Radiology and Surgery 13:917-924. DOI: 10.1007/s11548-018-1764-0.

Ren H, El-Khamy M, Lee J. 2017. Image Super Resolution Based on Fusing Multiple Convolution Neural Networks. In: IEEE Computer Society Conference on Computer Vision and Pattern Recognition Workshops. 54-61. DOI: 10.1109/CVPRW.2017.142.

Riegler G, Rüther M, Bischof H. 2016. ATGV-net: Accurate depth super-resolution. In: Lecture Notes in Computer Science (including subseries Lecture Notes in Artificial Intelligence and Lecture Notes in Bioinformatics). 268-284. DOI: 10.1007/978-3-319-46487-9_17.

Rouse DM, Hemami SS. 2008. Analyzing the role of visual structure in the recognition of natural image content with multi-scale SSIM. Human Vision and Electronic Imaging XIII 6806:680615. DOI: 10.1117/12.768060.

Rudin LI, Osher S, Fatemi E. 1992. Nonlinear total variation based noise removal algorithms. Physica D: Nonlinear Phenomena 60:259-268. DOI: 10.1016/0167-2789(92)90242-F.

Saad MA, Bovik AC, Charrier C. 2012. Blind image quality assessment: A natural scene statistics approach in the DCT domain. IEEE Transactions on Image Processing 21:33393352. DOI: 10.1109/TIP.2012.2191563.

Sajjadi MSM, Scholkopf B, Hirsch M. 2017. EnhanceNet: Single Image Super-Resolution Through Automated Texture Synthesis. In: Proceedings of the IEEE International Conference on Computer Vision. 4491-4500. DOI: 10.1109/ICCV.2017.481.

Sara U, Akter M, Uddin MS. 2019. Image Quality Assessment through FSIM, SSIM, MSE and PSNR - A Comparative Study. Journal of Computer and Communications 7:8-18. DOI: 10.4236/jcc.2019.73002.

Shaik KB, Ganesan P, Kalist V, Sathish BS, Jenitha JMM. 2015. Comparative Study of Skin Color Detection and Segmentation in HSV and YCbCr Color Space. Procedia Computer 
1743

1744

1745

1746

1747

1748

1749

1750

1751

1752

1753

1754

1755

1756

1757

1758

1759

1760

1761

1762

1763

1764

1765

1766

1767

1768

1769

1770

1771

1772

1773

1774

1775

1776

1777

1778

1779

1780

1781

1782

1783

1784

1785

1786

1787

1788

Science 57:41-48. DOI: 10.1016/j.procs.2015.07.362.

Shamsolmoali P, Zareapoor M, Jain DK, Jain VK, Yang J. 2018. Deep convolution network for surveillance records super-resolution. Multimedia Tools and Applications 78:23815-23829. DOI: $10.1007 / \mathrm{s} 11042-018-5915-7$.

Shermeyer J, Van Etten A. 2019. The effects of super-resolution on object detection performance in satellite imagery. In: IEEE Computer Society Conference on Computer Vision and Pattern Recognition Workshops. 0-0. DOI: 10.1109/CVPRW.2019.00184.

Shi W, Caballero J, Huszar F, Totz J, Aitken AP, Bishop R, Rueckert D, Wang Z. 2016. RealTime Single Image and Video Super-Resolution Using an Efficient Sub-Pixel Convolutional Neural Network. In: Proceedings of the IEEE Computer Society Conference on Computer Vision and Pattern Recognition. 1874-1883. DOI: 10.1109/CVPR.2016.207.

Shocher A, Cohen N, Irani M. 2018. Zero-Shot Super-Resolution Using Deep Internal Learning. In: Proceedings of the IEEE Computer Society Conference on Computer Vision and Pattern Recognition. 3118-3126. DOI: 10.1109/CVPR.2018.00329.

Simonyan K, Zisserman A. 2015. Very deep convolutional networks for large-scale image recognition. In: 3rd International Conference on Learning Representations, ICLR 2015 Conference Track Proceedings.

Sønderby CK, Caballero J, Theis L, Shi W, Huszár F. 2017. Amortised map inference for image super-resolution. In: 5th International Conference on Learning Representations, ICLR 2017 - Conference Track Proceedings. DOI: 10.17863/CAM.51994.

Song L, Zhang M, Wu X, He R. 2018. Adversarial discriminative heterogeneous face recognition. In: 32nd AAAI Conference on Artificial Intelligence, AAAI 2018.

Sroubek F, Cristobal G, Flusser J. 2008. Simultaneous super-resolution and blind deconvolution. In: Journal of Physics: Conference Series. 012048. DOI: 10.1088/1742-6596/124/1/012048.

Van Steenkiste G, Jeurissen B, Veraart J, Den Dekker AJ, Parizel PM, Poot DHJ, Sijbers J. 2016. Super-resolution reconstruction of diffusion parameters from diffusion-weighted images with different slice orientations. Magnetic Resonance in Medicine 75:181-195. DOI: $10.1002 / \mathrm{mrm} .25597$.

Sun D, Roth S, Black MJ. 2010. Secrets of optical flow estimation and their principles. In: Proceedings of the IEEE Computer Society Conference on Computer Vision and Pattern Recognition. 2432-2439. DOI: 10.1109/CVPR.2010.5539939.

Sun J, Xu Z, Shum HY. 2008. Image super-resolution using gradient profile prior. In: 26th IEEE Conference on Computer Vision and Pattern Recognition, CVPR. 1-8. DOI:

10.1109/CVPR.2008.4587659.

Szegedy C, Liu W, Jia Y, Sermanet P, Reed S, Anguelov D, Erhan D, Vanhoucke V, Rabinovich A. 2015. Going deeper with convolutions. In: Proceedings of the IEEE Computer Society Conference on Computer Vision and Pattern Recognition. 1-9. DOI: 10.1109/CVPR.2015.7298594.

Tai Y, Yang J, Liu X. 2017. Image super-resolution via deep recursive residual network. In: Proceedings - 30th IEEE Conference on Computer Vision and Pattern Recognition, CVPR 2017. 3147-3155. DOI: 10.1109/CVPR.2017.298.

Tai Y, Yang J, Liu X, Xu C. 2017. MemNet: A Persistent Memory Network for Image Restoration. In: Proceedings of the IEEE International Conference on Computer Vision. 4539-4547. DOI: 10.1109/ICCV.2017.486.

Taigman Y, Yang M, Ranzato M, Wolf L. 2014. DeepFace: Closing the gap to human-level performance in face verification. In: Proceedings of the IEEE Computer Society Conference 
1789

1790

1791

1792

1793

1794

1795

1796

1797

1798

1799

1800

1801

1802

1803

1804

1805

1806

1807

1808

1809

1810

1811

1812

1813

1814

1815

1816

1817

1818

1819

1820

1821

1822

1823

1824

1825

1826

1827

1828

1829

1830

1831

1832

1833

1834

on Computer Vision and Pattern Recognition. 1701-1708. DOI: 10.1109/CVPR.2014.220.

Talebi H, Milanfar P. 2018. NIMA: Neural Image Assessment. IEEE Transactions on Image Processing 27:3998-4011. DOI: 10.1109/TIP.2018.2831899.

Tan W, Yan B, Bare B. 2018. Feature Super-Resolution: Make Machine See More Clearly. In: Proceedings of the IEEE Computer Society Conference on Computer Vision and Pattern Recognition. 3994-4002. DOI: 10.1109/CVPR.2018.00420.

Tao X, Gao H, Liao R, Wang J, Jia J. 2017. Detail-Revealing Deep Video Super-Resolution. In: Proceedings of the IEEE International Conference on Computer Vision. 4472-4480. DOI: 10.1109/ICCV.2017.479.

Teh I, McClymont D, Carruth E, Omens J, McCulloch A, Schneider JE. 2020. Improved compressed sensing and super-resolution of cardiac diffusion MRI with structure-guided total variation. Magnetic Resonance in Medicine:1-13. DOI: 10.1002/mrm.28245.

Thapa D, Raahemifar K, Bobier WR, Lakshminarayanan V. 2016. A performance comparison among different super-resolution techniques. Computers and Electrical Engineering 54:313-329. DOI: 10.1016/j.compeleceng.2015.09.011.

Thung KH, Raveendran P. 2009. A survey of image quality measures. In: International Conference for Technical Postgraduates 2009, TECHPOS 2009. DOI: 10.1109/TECHPOS.2009.5412098.

Timofte R, Agustsson E, Gool L Van, Yang M-H, Zhang L, Lim B, Son S, Kim H, Nah S, Lee KM, Wang X, Tian Y, Yu K, Zhang Y, Wu S, Dong C, Lin L, Qiao Y, Loy CC, Bae W, Yoo J, Han Y, Ye JC, Choi J-S, Kim M, Fan Y, Yu J, Han W, Liu D, Yu H. 2017. NTIRE 2018 challenge on single image super-resolution: Methods and results. In: 2017 IEEE Conference on Computer Vision and Pattern Recognition Workshops (CVPRW). 11101121. DOI: 10.1109/CVPRW.2017.149.

Timofte R, De V, Gool L Van. 2013. Anchored neighborhood regression for fast example-based super-resolution. In: Proceedings of the IEEE International Conference on Computer Vision. 1920-1927. DOI: 10.1109/ICCV.2013.241.

Timofte R, Rothe R, Gool L Van. 2016. Seven Ways to Improve Example-Based Single Image Super Resolution. In: Proceedings of the IEEE Computer Society Conference on Computer Vision and Pattern Recognition. 1865-1873. DOI: 10.1109/CVPR.2016.206.

Timofte R, De Smet V, Van Gool L. 2015. A+: Adjusted anchored neighborhood regression for fast super-resolution. In: Lecture Notes in Computer Science (including subseries Lecture Notes in Artificial Intelligence and Lecture Notes in Bioinformatics). 111-126. DOI: 10.1007/978-3-319-16817-3_8.

Tipping ME, Bishop CM. 2003. Bayesian image super-resolution. In: Advances in Neural Information Processing Systems. 1303-1310.

Tong T, Li G, Liu X, Gao Q. 2017. Image Super-Resolution Using Dense Skip Connections. In: Proceedings of the IEEE International Conference on Computer Vision. 4799-4807. DOI: 10.1109/ICCV.2017.514.

Tsai R, Huang TS. 1984. Multiframe Image Restoration and Registration. Advances in Computer Vision and Image Processing 1:317-339.

Ulyanov D, Vedaldi A, Lempitsky V. 2020. Deep Image Prior. International Journal of Computer Vision. DOI: 10.1007/s11263-020-01303-4.

Vasu S, Thekke Madam N, Rajagopalan AN. 2019. Analyzing perception-distortion tradeoff using enhanced perceptual super-resolution network. In: Lecture Notes in Computer Science (including subseries Lecture Notes in Artificial Intelligence and Lecture Notes in

Peer] Comput. Sci. reviewing PDF | (CS-2021:02:58423:2:0:NEW 3 Jun 2021) 
1835

1836

1837

1838

1839

1840

1841

1842

1843

1844

1845

1846

1847

1848

1849

1850

1851

1852

1853

1854

1855

1856

1857

1858

1859

1860

1861

1862

1863

1864

1865

1866

1867

1868

1869

1870

1871

1872

1873

1874

1875

1876

1877

1878

1879

1880

Bioinformatics). DOI: 10.1007/978-3-030-11021-5_8.

Viswanathan M, Viswanathan M. 2005. Measuring speech quality for text-to-speech systems: Development and assessment of a modified mean opinion score (MOS) scale. Computer Speech and Language 19:55-83. DOI: 10.1016/j.csl.2003.12.001.

Vu T, Nguyen C V., Pham TX, Luu TM, Yoo CD. 2019. Fast and efficient image quality enhancement via desubpixel convolutional neural networks. In: Lecture Notes in Computer Science (including subseries Lecture Notes in Artificial Intelligence and Lecture Notes in Bioinformatics). DOI: 10.1007/978-3-030-11021-5_16.

Wang Z, Bovik AC, Sheikh HR, Simoncelli EP. 2004. Image quality assessment: From error visibility to structural similarity. IEEE Transactions on Image Processing 13:600-612. DOI: $10.1109 /$ TIP.2003.819861.

Wang Z, Chen J, Hoi SCHC. 2020. Deep Learning for Image Super-resolution: A Survey. IEEE Transactions on Pattern Analysis and Machine Intelligence:1-1. DOI: 10.1109/TPAMI.2020.2982166.

Wang Y, Perazzi F, McWilliams B, Sorkine-Hornung A, Sorkine-Hornung O, Schroers C. 2018a. A fully progressive approach to single-image super-resolution. In: IEEE Computer Society Conference on Computer Vision and Pattern Recognition Workshops. 864-873. DOI: 10.1109/CVPRW.2018.00131.

Wang Z, Simoncelli EP, Bovik AC. 2003. Multi-scale structural similarity for image quality assessment. In: The Thrity-Seventh Asilomar Conference on Signals, Systems \& Computers. 1398-1402. DOI: 10.1109/acssc.2003.1292216.

Wang L, Wang Y, Liang Z, Lin Z, Yang J, An W, Guo Y. 2019a. Learning parallax attention for stereo image super-resolution. In: Proceedings of the IEEE Computer Society Conference on Computer Vision and Pattern Recognition. 12250-12259. DOI: 10.1109/CVPR.2019.01253.

Wang X, Yu K, Dong C, Change Loy C. 2018b. Recovering Realistic Texture in Image SuperResolution by Deep Spatial Feature Transform. In: Proceedings of the IEEE Computer Society Conference on Computer Vision and Pattern Recognition. 606-615. DOI: 10.1109/CVPR.2018.00070.

Wang X, Yu K, Dong C, Tang X, Loy CC. 2019b. Deep network interpolation for continuous imagery effect transition. In: Proceedings of the IEEE Computer Society Conference on Computer Vision and Pattern Recognition. 1692-1701. DOI: 10.1109/CVPR.2019.00179. Wang X, Yu K, Wu S, Gu J, Liu Y. 2019c. ESRGAN : Enhanced Super-Resolution Generative Adversarial Networks. In: Leal-Taixé L, Roth S eds. Leal-Taixé L., Roth S. (eds) Computer Vision-ECCV 2018 Workshops. ECCV 2018. Springer, Cham, 63-79. DOI: https://doi.org/10.1007/978-3-030-11021-5_5.

Wei SE, Ramakrishna V, Kanade T, Sheikh Y. 2016. Convolutional pose machines. In: Proceedings of the IEEE Computer Society Conference on Computer Vision and Pattern Recognition. 4724-4732. DOI: 10.1109/CVPR.2016.511.

Wei Z, Yuan J, Cai Y. 1999. Picture quality evaluation method based on human perception. Tien Tzu Hsueh Pao/Acta Electronica Sinica 4.

Xiong Z, Sun X, Wu F. 2010. Robust web image/video super-resolution. IEEE Transactions on Image Processing 19:2017-2028. DOI: 10.1109/TIP.2010.2045707.

Xu X, Sun D, Pan J, Zhang Y, Pfister H, Yang MH. 2017. Learning to Super-Resolve Blurry Face and Text Images. In: Proceedings of the IEEE International Conference on Computer Vision. 251-260. DOI: 10.1109/ICCV.2017.36. 
1881

1882

1883

1884

1885

1886

1887

1888

1889

1890

1891

1892

1893

1894

1895

1896

1897

1898

1899

1900

1901

1902

1903

1904

1905

1906

1907

1908

1909

1910

1911

1912

1913

1914

1915

1916

1917

1918

1919

1920

1921

1922

1923

1924

1925

1926

Xue S, Qiu W, Liu F, Jin X. 2020. Wavelet-based residual attention network for image superresolution. Neurocomputing 382:116-126. DOI: 10.1016/j.neucom.2019.11.044.

Yang CY, Liu S, Yang MH. 2018. Hallucinating Compressed Face Images. International Journal of Computer Vision 126:597-614. DOI: 10.1007/s11263-017-1044-4.

Yang CY, Ma C, Yang MH. 2014. Single-image super-resolution: A benchmark. In: Lecture Notes in Computer Science (including subseries Lecture Notes in Artificial Intelligence and Lecture Notes in Bioinformatics). 372-386. DOI: 10.1007/978-3-319-10593-2_25.

Yang Y, Qi Y. 2021. Image super-resolution via channel attention and spatial graph convolutional network. Pattern Recognition 112:107798. DOI:

10.1016/j.patcog.2020.107798.

Yang J, Wright J, Huang T, Ma Y. 2008. Image super-resolution as sparse representation of raw image patches. In: 26th IEEE Conference on Computer Vision and Pattern Recognition, CVPR. 1-8. DOI: $10.1109 /$ CVPR.2008.4587647.

Yang J, Wright J, Huang TS, Ma Y. 2010. Image super-resolution via sparse representation. IEEE Transactions on Image Processing 19:2861-2873. DOI: 10.1109/TIP.2010.2050625.

Yang CY, Yang MH. 2013. Fast direct super-resolution by simple functions. In: Proceedings of the IEEE International Conference on Computer Vision. 561-568. DOI: 10.1109/ICCV.2013.75.

Yang Q, Yang R, Davis J, Nistér D. 2007. Spatial-depth super resolution for range images. In: Proceedings of the IEEE Computer Society Conference on Computer Vision and Pattern Recognition. 1-8. DOI: 10.1109/CVPR.2007.383211.

Yang W, Zhang X, Tian Y, Wang W, Xue JH, Liao Q. 2019. Deep Learning for Single Image Super-Resolution: A Brief Review. IEEE Transactions on Multimedia 21:3106-3121. DOI: 10.1109/TMM.2019.2919431.

Yao J, Liu G. 2018. Improved SSIM IQA of contrast distortion based on the contrast sensitivity characteristics of HVS. IET Image Processing 12:872-879. DOI: 10.1049/ietipr.2017.0209.

Yu X, Fernando B, Hartley R, Porikli F. 2018. Super-Resolving Very Low-Resolution Face Images with Supplementary Attributes. In: Proceedings of the IEEE Computer Society Conference on Computer Vision and Pattern Recognition. 908-917. DOI: 10.1109/CVPR.2018.00101.

Yu F, Koltun V. 2016. Multi-scale context aggregation by dilated convolutions. In: 4th International Conference on Learning Representations, ICLR 2016 - Conference Track Proceedings.

Yu X, Porikli F. 2016. Ultra-resolving face images by discriminative generative networks. In: Lecture Notes in Computer Science (including subseries Lecture Notes in Artificial Intelligence and Lecture Notes in Bioinformatics). 318-333. DOI: 10.1007/978-3-31946454-1_20.

Yu X, Porikli F. 2017. Face hallucination with tiny unaligned images by transformative discriminative neural networks. In: 31st AAAI Conference on Artificial Intelligence, AAAI 2017.

Yuan Y, Liu S, Zhang J, Zhang Y, Dong C, Lin L. 2018. Unsupervised image super-resolution using cycle-in-cycle generative adversarial networks. In: IEEE Computer Society Conference on Computer Vision and Pattern Recognition Workshops. 701-710. DOI: 10.1109/CVPRW.2018.00113.

Zaitoun NM, Aqel MJ. 2015. Survey on Image Segmentation Techniques. Procedia Computer

Peer] Comput. Sci. reviewing PDF | (CS-2021:02:58423:2:0:NEW 3 Jun 2021) 
1927

1928

1929

1930

1931

1932

1933

1934

1935

1936

1937

1938

1939

1940

1941

1942

1943

1944

1945

1946

1947

1948

1949

1950

1951

1952

1953

1954

1955

1956

1957

1958

1959

1960

1961

1962

1963

1964

1965

1966

1967

1968

1969

1970

1971

1972

Science 65:797-806. DOI: 10.1016/j.procs.2015.09.027.

Zeiler MD, Krishnan D, Taylor GW, Fergus R. 2010. Deconvolutional networks. In: Proceedings of the IEEE Computer Society Conference on Computer Vision and Pattern Recognition. 2528-2535. DOI: 10.1109/CVPR.2010.5539957.

Zhang YN, An MQ. 2017. Deep Learning- and Transfer Learning-Based Super Resolution Reconstruction from Single Medical Image. Journal of Healthcare Engineering 2017. DOI: $10.1155 / 2017 / 5859727$.

Zhang X, Chen Q, Ng R, Koltun V. 2019a. Zoom to learn, learn to zoom. In: Proceedings of the IEEE Computer Society Conference on Computer Vision and Pattern Recognition. 37623770. DOI: 10.1109/CVPR.2019.00388.

Zhang Y, Li K, Li K, Wang L, Zhong B, Fu Y. 2018a. Image super-resolution using very deep residual channel attention networks. In: Lecture Notes in Computer Science (including subseries Lecture Notes in Artificial Intelligence and Lecture Notes in Bioinformatics). DOI: 10.1007/978-3-030-01234-2_18.

Zhang Y, Li K, Li K, Zhong B, Fu Y. 2019b. Residual non-local attention networks for image restoration. In: 7th International Conference on Learning Representations, ICLR 2019.

Zhang Y, Tian Y, Kong Y, Zhong B, Fu Y. 2018b. Residual Dense Network for Image SuperResolution. In: Proceedings of the IEEE Computer Society Conference on Computer Vision and Pattern Recognition. 2472-2481. DOI: 10.1109/CVPR.2018.00262.

Zhang H, Wang P, Zhang C, Jiang Z. 2019c. A comparable study of CNN-based single image super-resolution for space-based imaging sensors. Sensors (Switzerland) 19:3234. DOI: $10.3390 / \mathrm{s} 19143234$.

Zhang K, Zhang Z, Cheng CW, Hsu WH, Qiao Y, Liu W, Zhang T. 2018c. Super-Identity Convolutional Neural Network for Face Hallucination. In: Proceedings of the European Conference on Computer Vision (ECCV). 183-198. DOI: 10.1007/978-3-030-01252-6_12.

Zhang L, Zhang L, Mou X, Zhang D. 2011. FSIM: A feature similarity index for image quality assessment. IEEE Transactions on Image Processing 20:2378-2386. DOI: 10.1109/TIP.2011.2109730.

Zhang L, Zhang H, Shen H, Li P. 2010. A super-resolution reconstruction algorithm for surveillance images. Signal Processing 90:848-859. DOI: 10.1016/j.sigpro.2009.09.002.

Zhang K, Zuo W, Zhang L. 2018. Learning a Single Convolutional Super-Resolution Network for Multiple Degradations. In: 2018 IEEE/CVF Conference on Computer Vision and Pattern Recognition. 3262-3271. DOI: 10.1109/CVPR.2018.00344.

Zhao H, Gallo O, Frosio I, Kautz J. 2016. Loss Functions for Image Restoration With Neural Networks. IEEE Transactions on Computational Imaging 3:47-57. DOI: 10.1109/tci.2016.2644865.

Zhao H, Shi J, Qi X, Wang X, Jia J. 2017. Pyramid scene parsing network. In: Proceedings 30th IEEE Conference on Computer Vision and Pattern Recognition, CVPR 2017. 28812890. DOI: $10.1109 /$ CVPR.2017.660.

Zhou L, Feng S. 2019. A Review of Deep Learning for Single Image Super-Resolution. In: ICIIBMS 2019 - 4th International Conference on Intelligent Informatics and Biomedical Sciences. 139-142. DOI: 10.1109/ICIIBMS46890.2019.8991477.

Zhu X, Li Z, Lou J, Shen Q. 2021. Video super-resolution based on a spatio-temporal matching network. Pattern Recognition 110:107619. DOI: 10.1016/j.patcog.2020.107619.

Zhu S, Liu S, Loy CC, Tang X. 2016. Deep cascaded Bi-network for face hallucination. In: Proceedings of the European Conference on Computer Vision (ECCV). 614-630. DOI:

Peer] Comput. Sci. reviewing PDF | (CS-2021:02:58423:2:0:NEW 3 Jun 2021) 
1973

1974

1975

1976

1977

1978

1979

1980

1981

1982

1983

1984

1985

1986
10.1007/978-3-319-46454-1_37.

Zhu JY, Park T, Isola P, Efros AA. 2017a. Unpaired Image-to-Image Translation Using CycleConsistent Adversarial Networks. In: Proceedings of the IEEE International Conference on Computer Vision. 2223-2232. DOI: 10.1109/ICCV.2017.244.

Zhu H, Tang X, Xie J, Song W, Mo F, Gao X. 2018. Spatio-temporal super-resolution reconstruction of remote-sensing images based on adaptive multi-scale detail enhancement. Sensors (Switzerland) 18:498. DOI: 10.3390/s18020498.

Zhu XX, Tuia D, Mou L, Xia G-S, Zhang L, Xu F, Fraundorfer F. 2017b. Deep Learning in Remote Sensing: A Comprehensive Review and List of Resources. IEEE Geoscience and Remote Sensing Magazine 5:8-36. DOI: 10.1109/MGRS.2017.2762307.

Zomet A, Rav-Acha A, Peleg S. 2001. Robust super-resolution. In: Proceedings of the IEEE Computer Society Conference on Computer Vision and Pattern Recognition. I-I. DOI: 10.1109/cvpr.2001.990535. 


\section{Figure 1}

Hierarchical classification of this survey.

Four main categories are (a) classical methods of image super-resolution, (b) deep learningbased methods for SR, (c) applications of super-resolution, (d) future research and directions in SR. Green color represent first-level sections, the blue color is for second-level subsections, and orange color represent third level subsections.

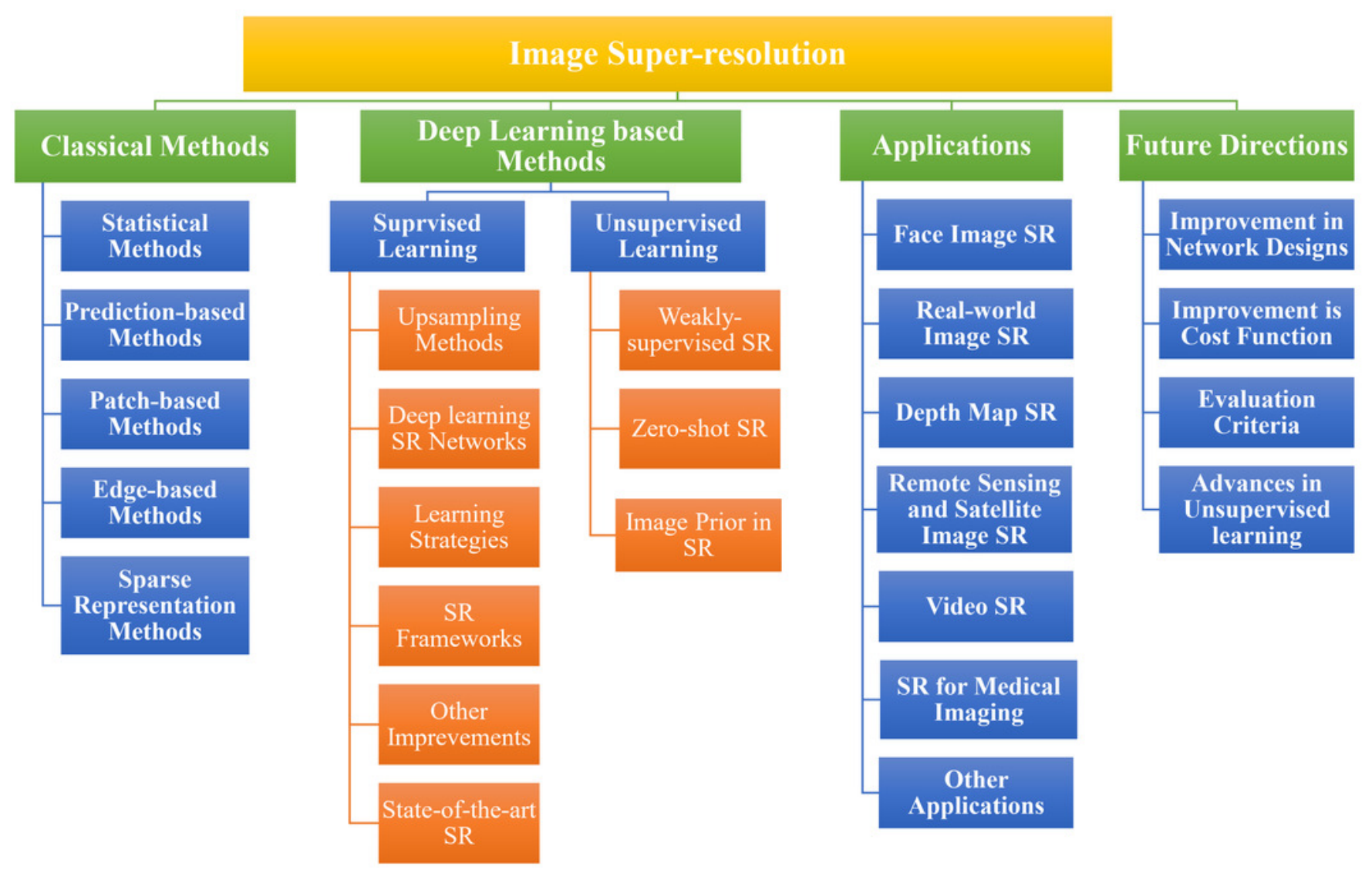


Figure 2

Downsampling and upsampling in super-resolution.

Noise is added to simulate realistic degradation within an image.

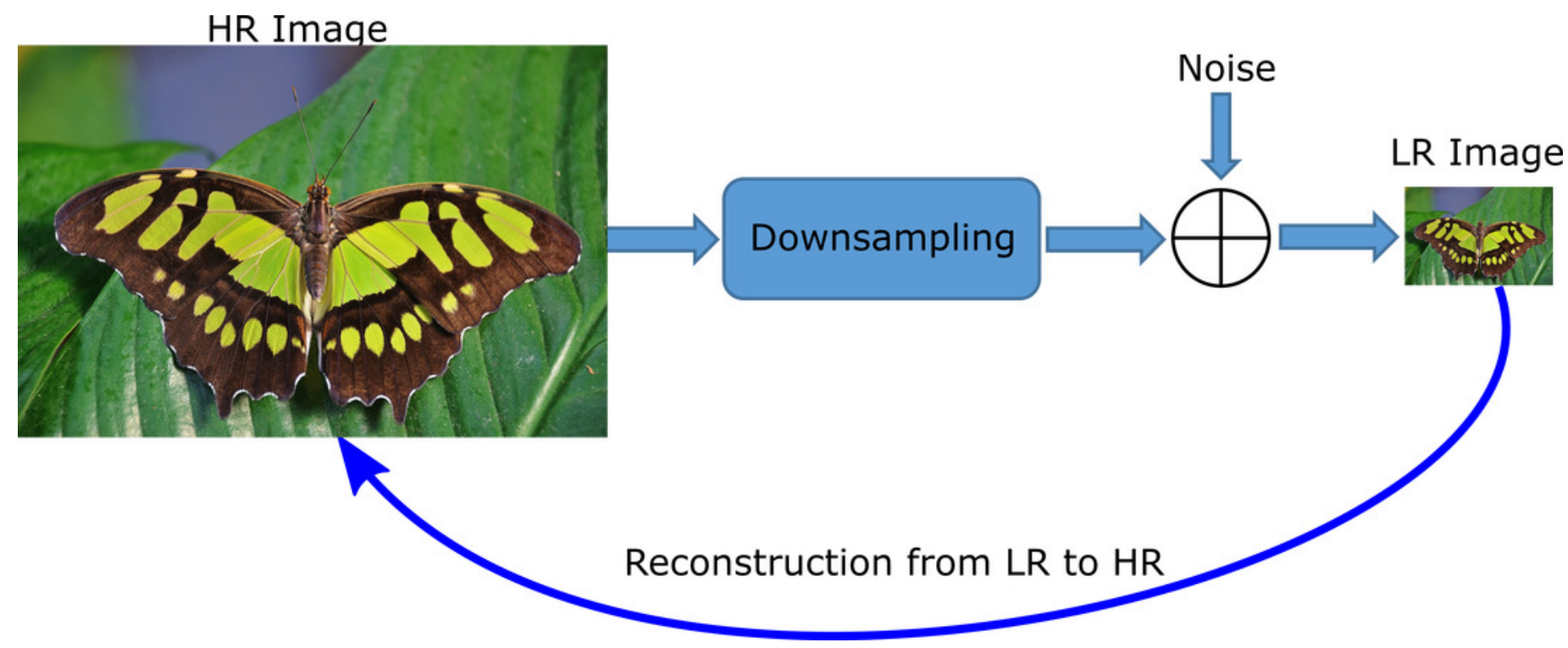


Figure 3

Methodology for the collection of studies.

Sample details based on inclusion/exclusion criteria defined in Table 5. 
Total articles identified using databases,

$$
\mathrm{n}=653
$$

\section{Google scholar $(\mathrm{n}=538)$ IEEE Xplore $(n=76)$ Science Direct $(\mathrm{n}=39)$}

\section{Articles after initial} screening and duplicate removal

$$
\mathrm{n}=195
$$

Articles identified from bibliography of already searched papers

$$
\mathrm{n}=47
$$

\section{Total articles identified} using databases,

$$
\mathrm{n}=242
$$


Figure 4

Sub-pixel layer. Blue color represents the input convolution, and output feature maps are represented in other colors.

(a) Input (b) Convolution (c) Reshaping

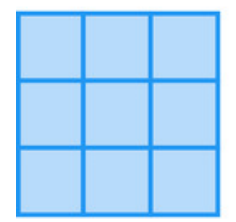

(a)

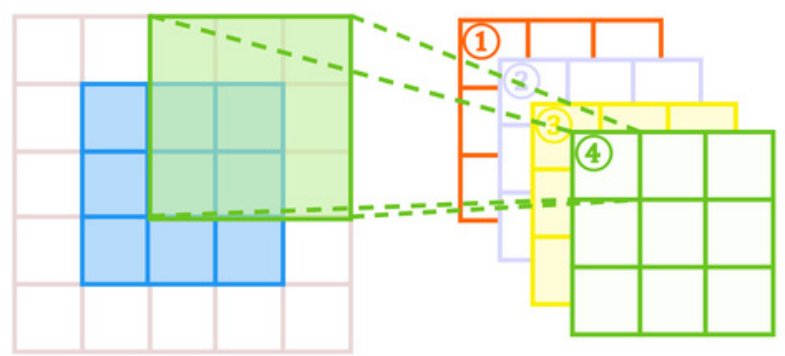

(b)

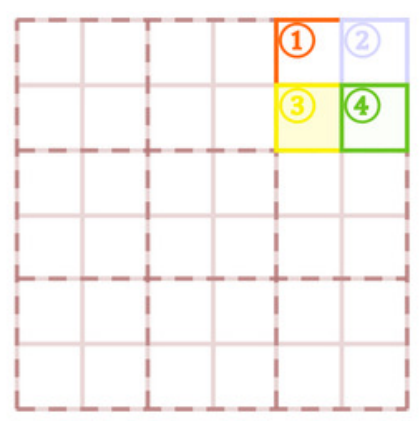

(c) 
Figure 5

Deconvolution layer. The blue color represents the input, and the green color represents the convolution operation.

(a) Input (b) Expansion (c) Convolution

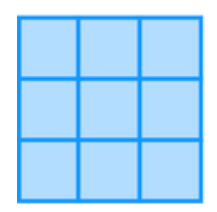

(a)
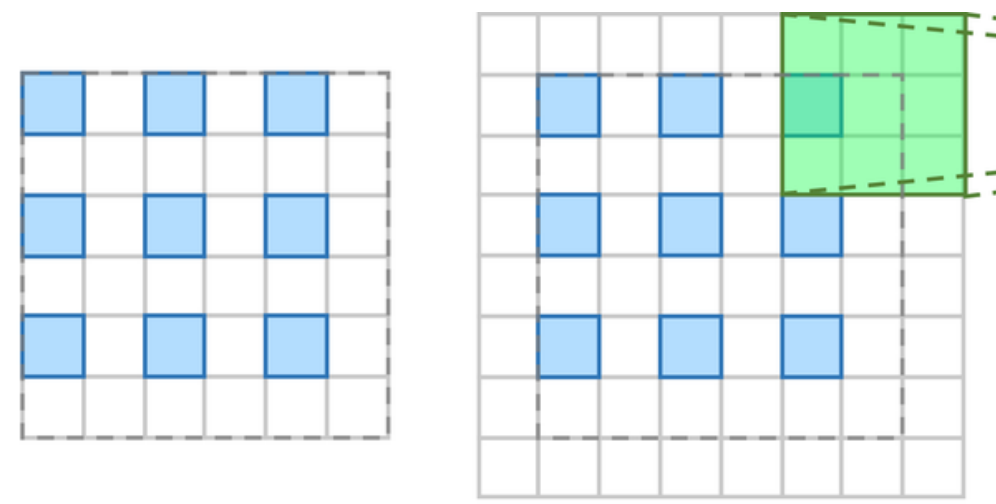

(b)

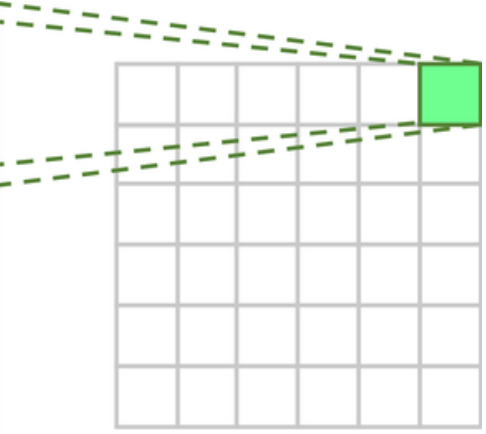

(c) 


\section{Figure 6}

Deep-learning network structures for super-resolution.

(a) recursive learning, (b) residual learning, (c) dense connection-based learning, (d) multiscale learning, (e) advanced convolution-based learning, (f) attention-based learning

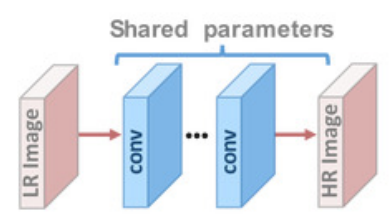

(a)

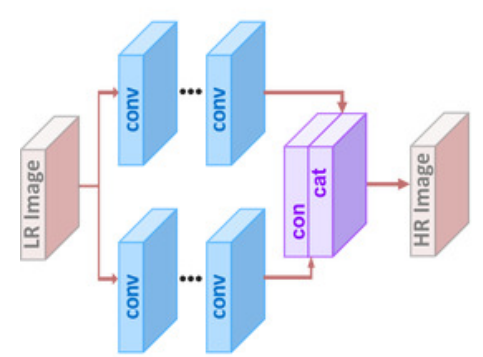

(d)

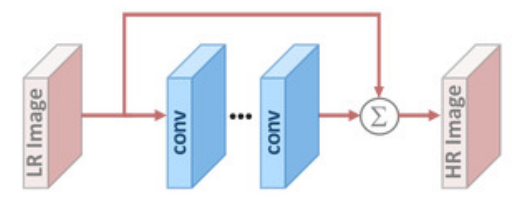

(b)

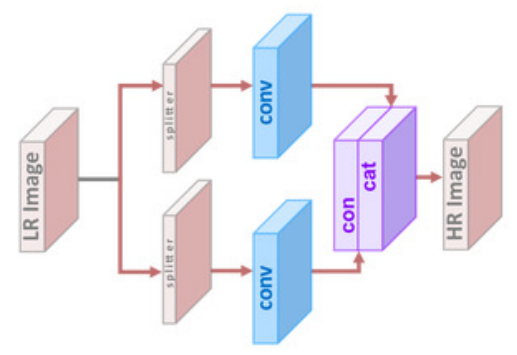

(e)

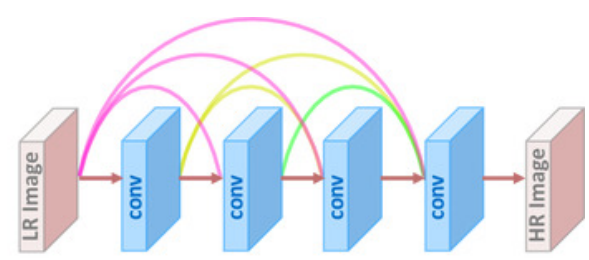

(c)

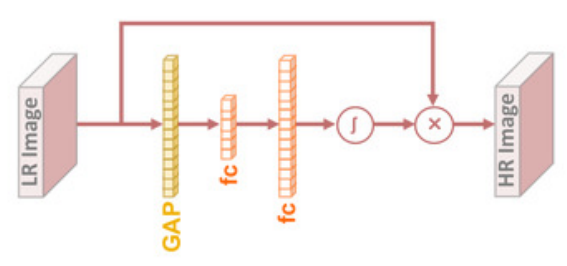

(f) 
Figure 7

Pre-upsampling-based super-resolution network pipeline.

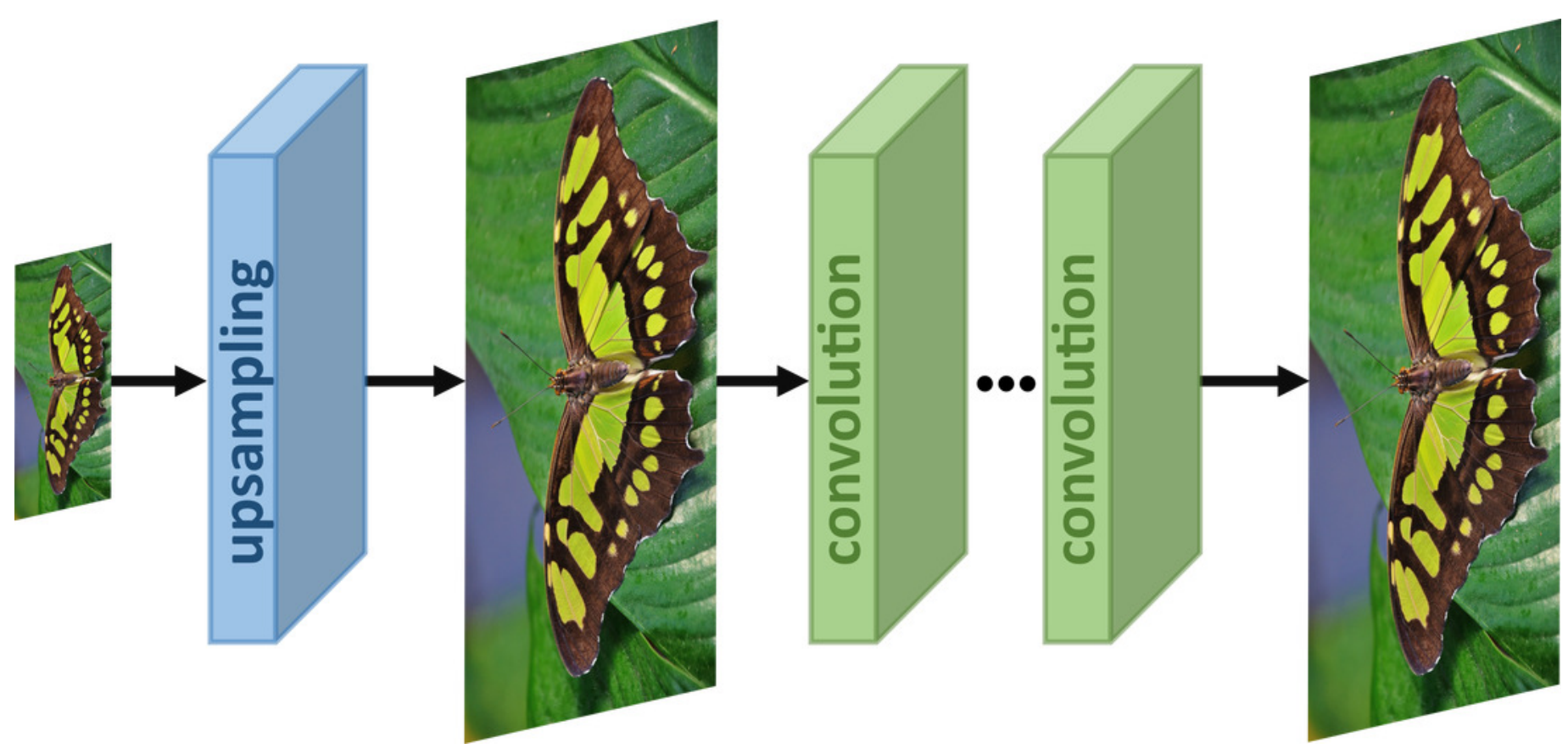




\section{Figure 8}

Post-upsampling-based super-resolution network pipeline.

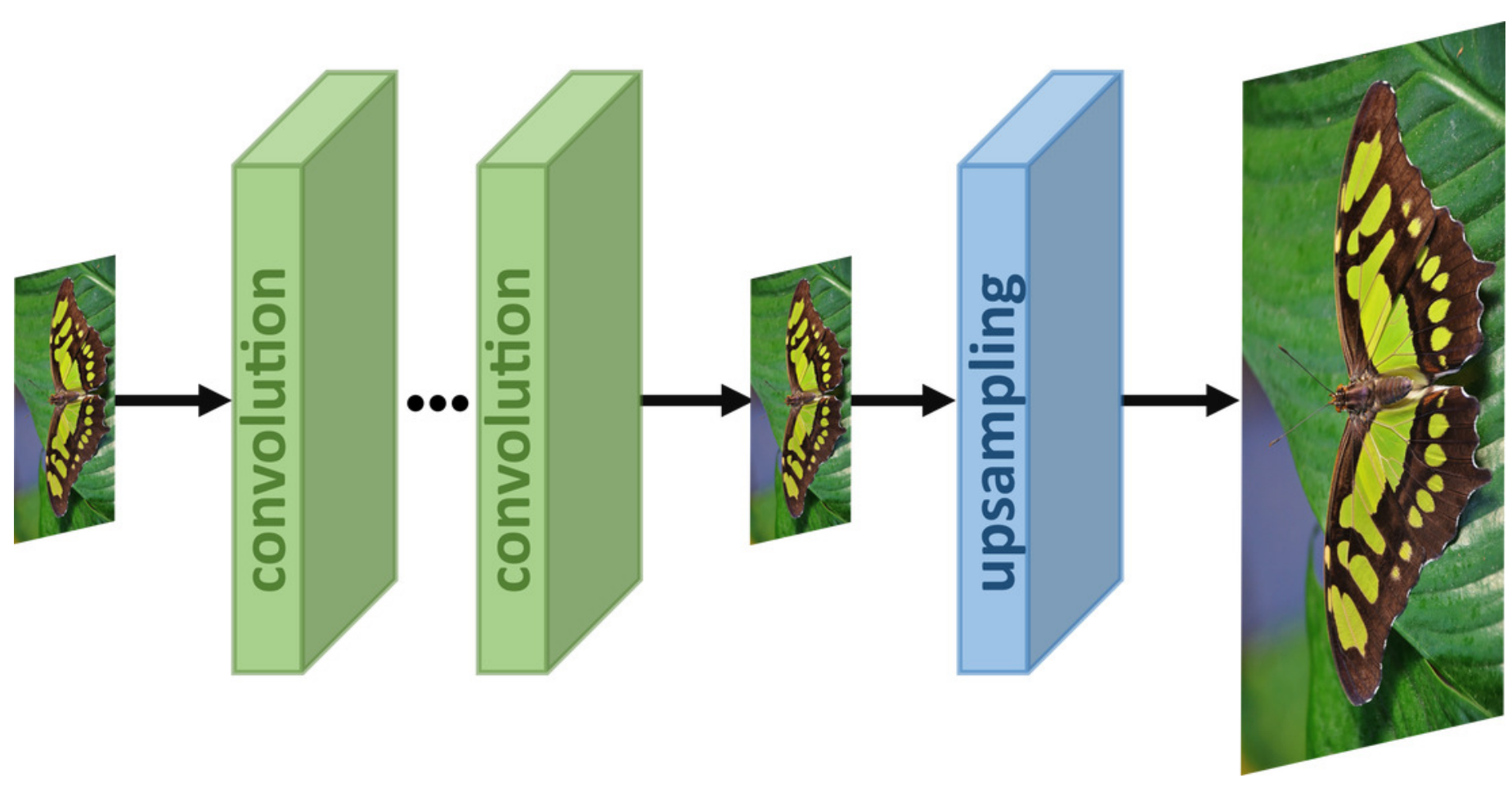


Figure 9

Iterative up-and-down sampling-based super-resolution network pipeline.

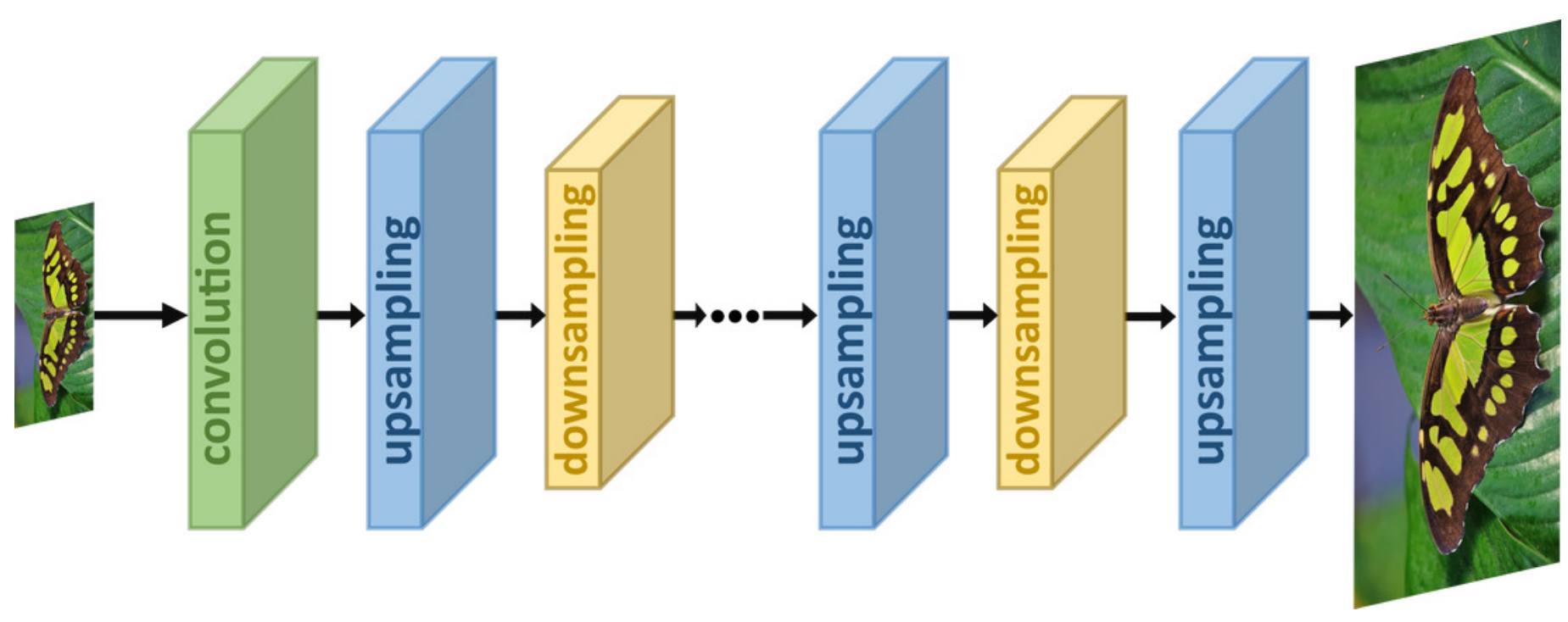


Figure 10

Progressive sampling-based super-resolution network pipeline.

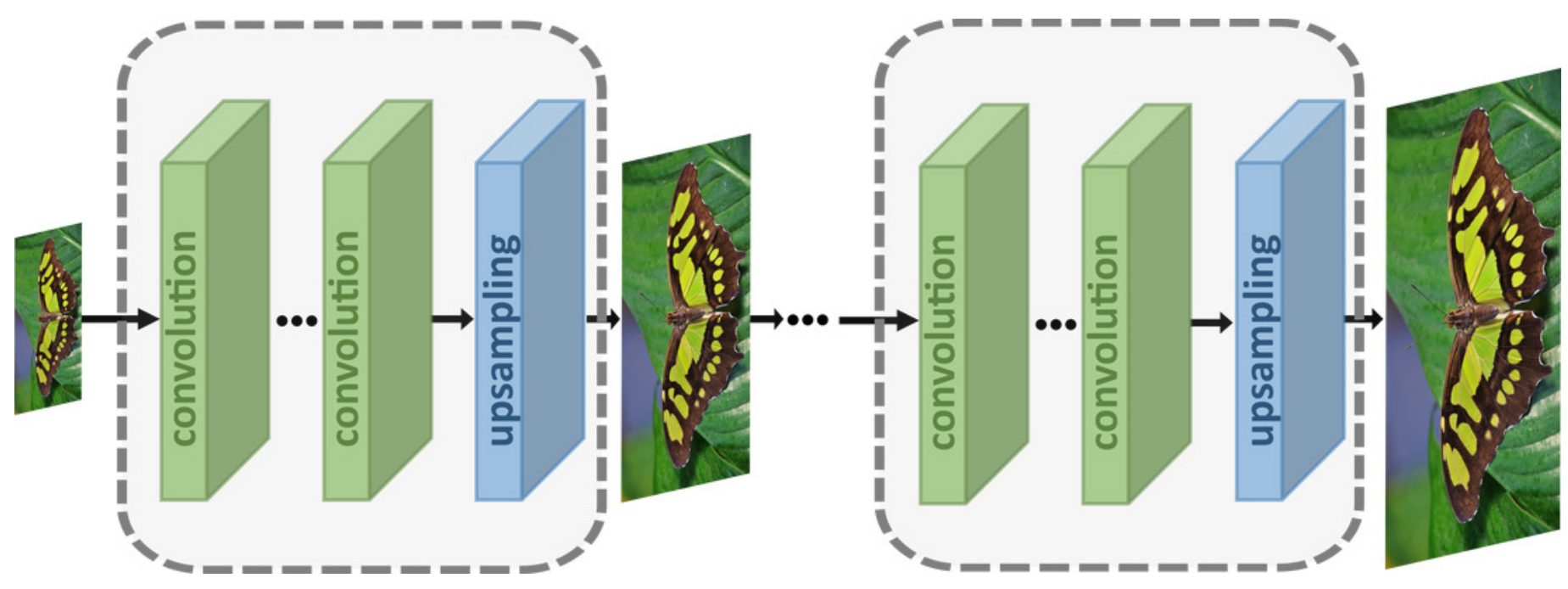


Figure 11

Benchmarking of super-resolution models.

Image quality index is represented by PSNR (in blue color), which is a significant evaluation indicator of any super-resolution method; the total number of parameters learned by every method is shown in green. The computational efficiency is measured in tera multiply-adds, and it is shown in orange color.

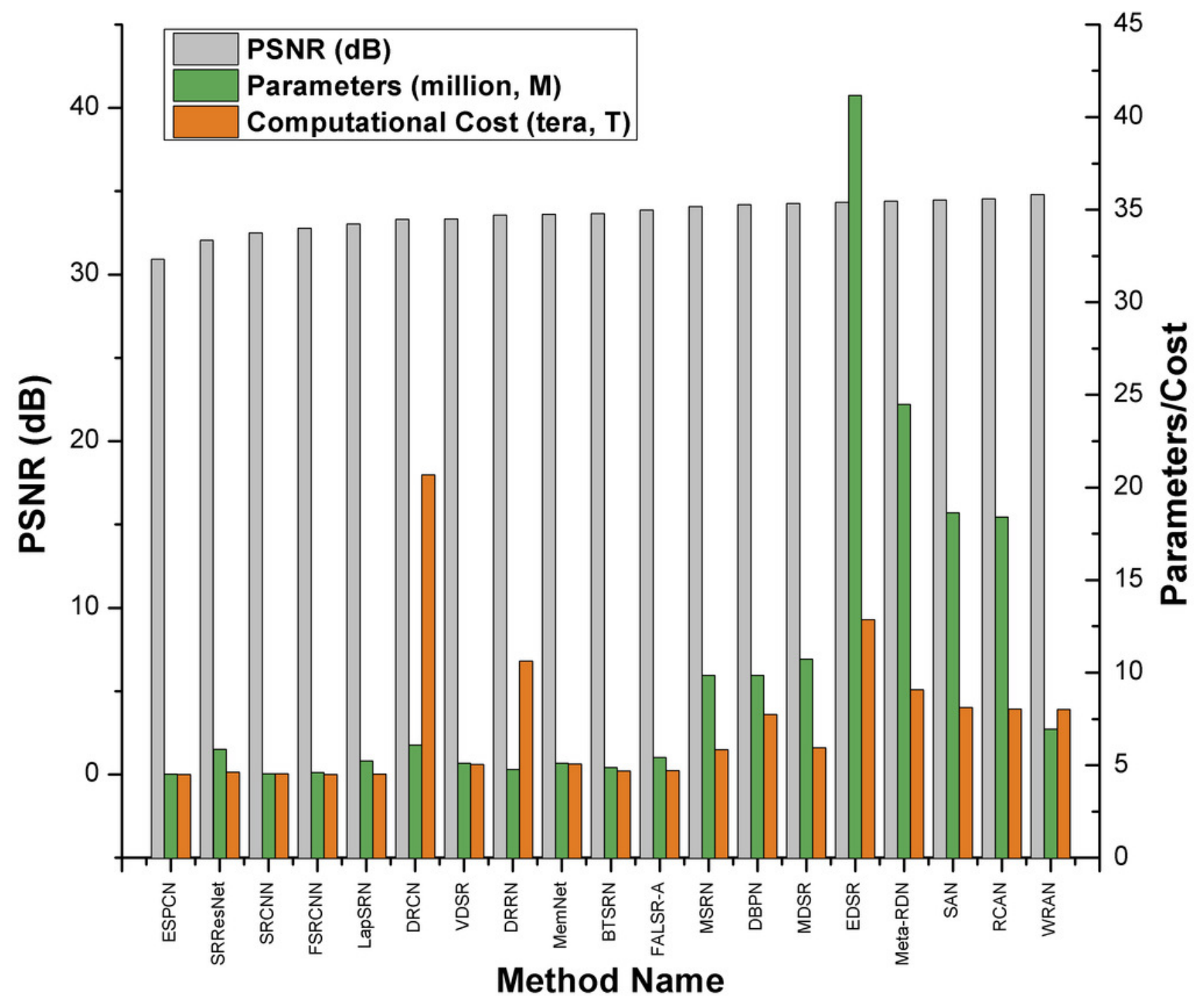




\section{Table $\mathbf{1}$ (on next page)}

Comparison of image quality metrics for super-resolution. 


\begin{tabular}{|c|c|c|}
\hline Method & Strengths & Weaknesses \\
\hline PSNR & $\begin{array}{l}\text { - Most commonly used quality } \\
\text { assessment metric; thus, it is } \\
\text { easy to compare results with } \\
\text { other methods. } \\
\text { - Quantitative and is based on } \\
\text { MSE }\end{array}$ & $\begin{array}{l}\text { - Since this metric is pixel-based, the } \\
\text { overall score could be misleading in } \\
\text { some cases where two images could } \\
\text { be visually different, but the PSNR } \\
\text { would still be high. } \\
\text { - This method does not give consider } \\
\text { the structural information within the } \\
\text { image }\end{array}$ \\
\hline SSIM & $\begin{array}{l}\text { After PSNR, this metric is the } \\
\text { most commonly used IQA } \\
\text { metric; thus, comparing results } \\
\text { with other methods is easier. } \\
\text { Quantitatively scores an image } \\
\text { based on its structural similarity } \\
\text { with the original image with the } \\
\text { possibility to change the weights } \\
\text { of luminance, contrast, and } \\
\text { structural comparison. }\end{array}$ & $\begin{array}{l}\text { - SSIM is unstable in cases where } \\
\text { the variance or luminance of the } \\
\text { reference image is low; thus, in } \\
\text { medical imaging, this metric } \\
\text { could give inconsistent results. }\end{array}$ \\
\hline $\begin{array}{l}\text { Opinion } \\
\text { Scoring }\end{array}$ & $\begin{array}{l}\text { Opinion scoring is a subjective } \\
\text { quality metric, and human } \\
\text { testers grade image quality } \\
\text { based on predefined parameters } \\
\text { such as sharpness, color, and } \\
\text { natural look. } \\
\text { This method is particularly } \\
\text { suitable for human face } \\
\text { reconstruction methods. }\end{array}$ & $\begin{array}{l}\text { Limitations include non-linear } \\
\text { scoring among the testers, human } \\
\text { error, and changes in test parameters. } \\
\text { - Scoring takes much time, especially } \\
\text { for large datasets }\end{array}$ \\
\hline $\begin{array}{l}\text { Perceptual } \\
\text { Quality }\end{array}$ & $\begin{array}{l}\text { This method is similar to } \\
\text { opinion scoring, but human } \\
\text { testers are replaced by models } \\
\text { that learn the behavior of testers } \\
\text { using deep learning. } \\
\text { - Very fast compared to opinion } \\
\text { scoring. }\end{array}$ & $\begin{array}{l}\text { Requires additional resources for } \\
\text { training the network to learn the } \\
\text { features for the quality assessment } \\
\text { network. } \\
\text { - It depends on annotated datasets to } \\
\text { learn human behavior. }\end{array}$ \\
\hline $\begin{array}{l}\text { Task-based } \\
\text { Evaluation }\end{array}$ & $\begin{array}{l}\text { This metric is appropriate if the } \\
\text { SR images are used to perform } \\
\text { another task, for example, object } \\
\text { detection/classification and } \\
\text { diagnosis. } \\
\text { - It helps in measuring the } \\
\text { performance of the whole task, } \\
\text { which uses SR images. }\end{array}$ & $\begin{array}{l}\text { - Highly dependent on the } \\
\text { performance of the associated task. } \\
\text { - Same SR images will give different } \\
\text { scores if there is a change in task } \\
\text { parameters. }\end{array}$ \\
\hline
\end{tabular}




\section{Table 2 (on next page)}

List of benchmark datasets used in super-resolution. 


\begin{tabular}{|c|c|c|c|c|c|}
\hline Name & $\begin{array}{c}\text { Number of } \\
\text { images/ } \\
\text { pairs }\end{array}$ & $\begin{array}{l}\text { Image } \\
\text { format }\end{array}$ & Type & Resolution & Details of images \\
\hline BSD100 (Martin et al., 2001) & 100 & PNG & Unpaired & $(480,320)$ & $\begin{array}{l}100 \text { images of animals, people, buildings, } \\
\text { scenic views etc. }\end{array}$ \\
\hline BSDS300 (Martin et al., 2001) & 300 & JPG & Unpaired & $(430,370)$ & $\begin{array}{l}300 \text { images of animals, people, buildings, } \\
\text { scenic views, plants, etc. }\end{array}$ \\
\hline BSDS500 (Arbeláez et al., 2010) & 500 & JPG & Unpaired & $(430,370)$ & $\begin{array}{l}\text { Extended version of BSD } 300 \text { with additional } \\
200 \text { images }\end{array}$ \\
\hline CelebA (Liu et al., 2015) & 202,599 & PNG & Unpaired & $\begin{array}{l}(2048, \\
1024)\end{array}$ & $\begin{array}{l}\text { Over } 40 \text { attribute defined categories of } \\
\text { celebrities }\end{array}$ \\
\hline DIV2K (Agustsson \& Timofte, 2017) & 1000 & PNG & Paired & $\begin{array}{l}(2048, \\
1024)\end{array}$ & Objects, People, Animals, scenery, nature \\
\hline Manga109 (Fujimoto et al., 2016) & 109 & PNG & Unpaired & $(800,1150)$ & $\begin{array}{l}109 \text { manga volumes drawn by professional } \\
\text { manga artists in Japan }\end{array}$ \\
\hline MS-COCO (Lin et al., 2014) & 164,000 & JPG & Unpaired & $(640,480)$ & Labeled objects with over 80 object categories \\
\hline OutdoorScene (Wang et al., 2018) & 10624 & PNG & Unpaired & $(550,450)$ & $\begin{array}{l}\text { Outdoor scenes including plants, animals, } \\
\text { sceneries, water reservoirs, etc. }\end{array}$ \\
\hline PIRM (Blau et al., 2018) & 200 & PNG & Unpaired & $(600,500)$ & Sceneries, people, flowers, etc. \\
\hline Set14 (Zeyde, Elad \& Protter, 2012) & 14 & PNG & Unpaired & $(500,450)$ & $\begin{array}{l}\text { Faces, animals, flowers, animated characters, } \\
\text { insects, etc. }\end{array}$ \\
\hline Set5 (Bevilacqua et al., 2012) & 5 & PNG & Unpaired & $(300,340)$ & $\begin{array}{l}\text { Only } 5 \text { images including, butterfly, baby, bird, } \\
\text { head, and women. }\end{array}$ \\
\hline T91 (Yang et al., 2010) & 91 & PNG & Unpaired & $(250,200)$ & 91 images of fruits, cars, faces, etc. \\
\hline $\begin{array}{l}\text { Urban100 (Huang, Singh \& Ahuja, } \\
\text { 2015) }\end{array}$ & 100 & PNG & Unpaired & $(1000,800)$ & Urban buildings, architecture \\
\hline VOC2012 (Everingham et al., 2014) & 11,530 & JPG & Unpaired & $(500,400)$ & Labelled objects with over 20 classes \\
\hline
\end{tabular}




\section{Table 3 (on next page)}

Inclusion and exclusion criteria. 


\begin{tabular}{|c|c|c|}
\hline Section & Inclusion & Exclusion \\
\hline Introduction & $\begin{array}{l}\text { Methods that defined image } \\
\text { interpolation and performed some } \\
\text { practical form of image } \\
\text { interpolation, i.e., super- } \\
\text { resolution }\end{array}$ & $\begin{array}{l}\text { - Studies that solely defined } \\
\text { model } \\
\text { - } \quad \text { Review articles }\end{array}$ \\
\hline Classical Methods & $\begin{array}{l}\text { Methods that performed pixel, } \\
\text { neighborhood, or any classical } \\
\text { image interpolation }\end{array}$ & $\begin{array}{l}\text { Application research where } \\
\text { applications of classical } \\
\text { methods were discussed } \\
\text { - Review papers }\end{array}$ \\
\hline $\begin{array}{l}\text { Deep learning-based } \\
\text { methods }\end{array}$ & $\begin{array}{l}\text { Development of image super- } \\
\text { resolution using deep learning } \\
\text { methods, including review papers }\end{array}$ & $\begin{array}{l}\text { Papers that emphasize video } \\
\text { super-resolution as these } \\
\text { papers give priority to } \\
\text { frame per second (FPS) and } \\
\text { inference time were not } \\
\text { included }\end{array}$ \\
\hline Applications & $\begin{array}{l}\text { Direct applications of super- } \\
\text { resolution methods in the six } \\
\text { fields defined in Section } 7 \text { were } \\
\text { included }\end{array}$ & $\begin{array}{l}\text { - Applications that combined } \\
\text { other methods with image } \\
\text { super-resolution and SR } \\
\text { was a limited part were not } \\
\text { included } \\
\text { - Review papers }\end{array}$ \\
\hline
\end{tabular}




\section{Table 4 (on next page)}

Comparison of upsampling methods. 


\begin{tabular}{|c|c|c|}
\hline Method & Strengths & Weaknesses \\
\hline $\begin{array}{l}\text { Sub-pixel } \\
\text { layer }\end{array}$ & $\begin{array}{l}\text { - It uses convolution in an end- } \\
\text { to-end manner, so it is } \\
\text { frequently used in SR models. } \\
\text { - This layer has a wide receptive } \\
\text { field, which helps learn more } \\
\text { contextual information }\end{array}$ & $\begin{array}{l}\text { This layer may generate some } \\
\text { false artifacts at the boundaries of } \\
\text { complex patterns due to its uneven } \\
\text { distribution of the respective field. } \\
\text { - Upscaling factor is fixed }\end{array}$ \\
\hline $\begin{array}{l}\text { Deconvolution } \\
\text { layer }\end{array}$ & $\begin{array}{l}\text { This layer is most commonly } \\
\text { used in SR methods, and it } \\
\text { generates HR images in an end- } \\
\text { to-end manner. } \\
\text { - Compatible with vanilla } \\
\text { convolution }\end{array}$ & $\begin{array}{l}\text { - In some cases, due to uneven } \\
\text { overlapping within the generated } \\
\text { HR image, the patterns are } \\
\text { replicated in a check-like format } \\
\text { and may result in a non-realistic } \\
\text { HR image. } \\
\text { - Upscaling factor is fixed }\end{array}$ \\
\hline $\begin{array}{l}\text { Meta } \\
\text { upscaling }\end{array}$ & $\begin{array}{l}\text { This method uses arbitrary } \\
\text { scaling factors to generate the } \\
\text { SR image. } \\
\text { Extracts more information from } \\
\text { the LR feature maps, which } \\
\text { helps to construct an HR image } \\
\text { using meta upscaling in the last } \\
\text { layer of the SR models, which } \\
\text { makes this method an end-to- } \\
\text { end SR approach }\end{array}$ & $\begin{array}{l}\text { The whole process may become } \\
\text { unstable for high-scale factors as it } \\
\text { predicts the convolution weights } \\
\text { for every single pixel independent } \\
\text { of the image information within } \\
\text { those pixels. }\end{array}$ \\
\hline
\end{tabular}




\section{Table 5 (on next page)}

SR method details of various SR algorithms

“US," “Rec.," "Res.," "Attent.," “Dense," "Pre.," "Post.," “Iter.," and "Prog." represent upsampling methods, recursive learning, residual learning, attention-based learning, dense connections, pre-upsampling framework, post-upsampling framework, iterative up-down upsampling framework, and progressive upsampling framework respectively. 


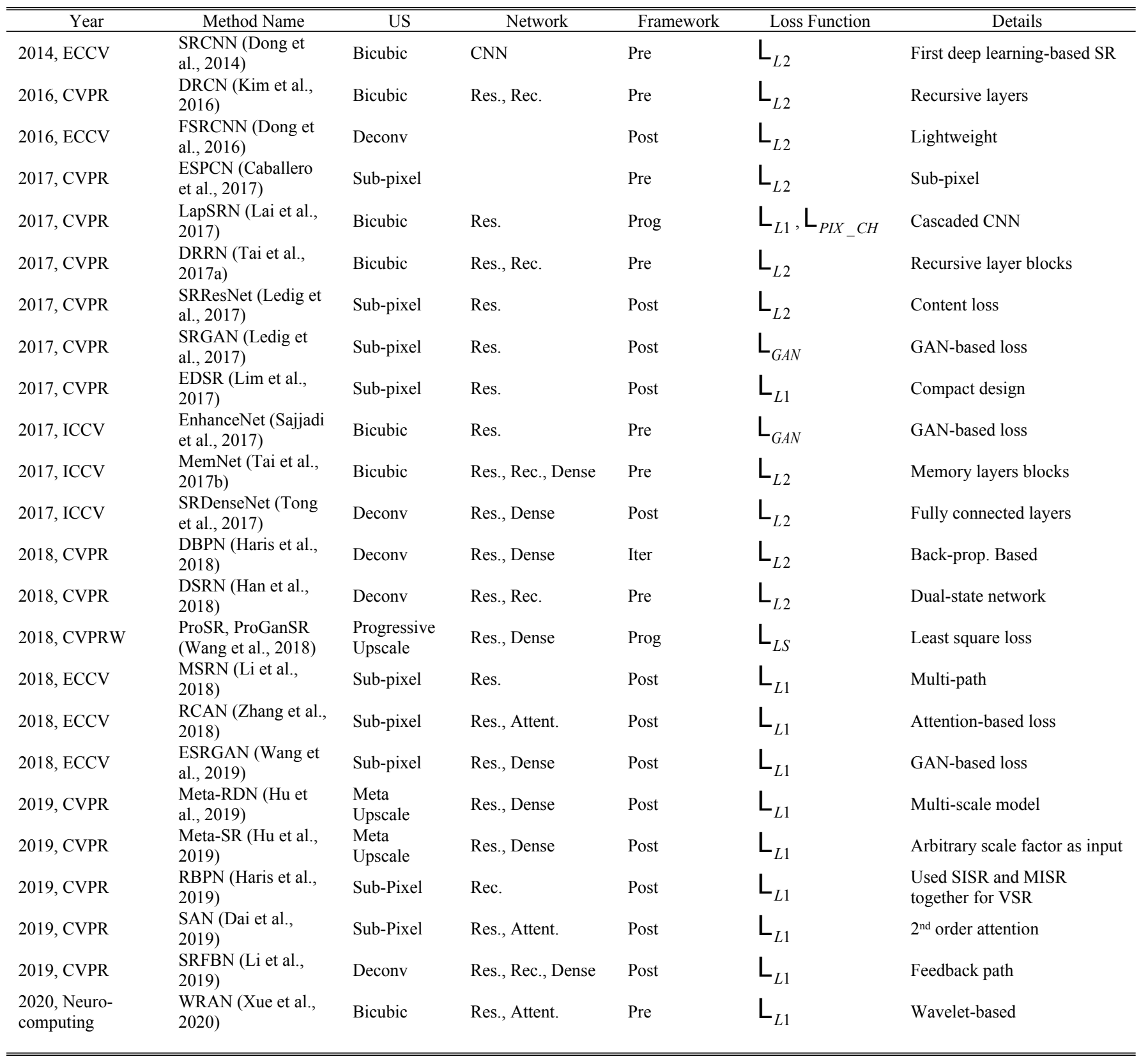

